\title{
A Seepage Investigation of an Area at and near Oak Ridge National Laboratory, Oak Ridge, Tennessee, March through August 1993
}

By GREGORY C. JOHNSON

U.S. GEOLOGICAL SURVEY

Open-File Report 95-442

Prepared in cooperation with the U.S. DEPARTMENT OF ENERGY

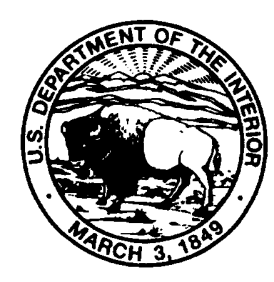




\title{
U.S. DEPARTMENT OF THE INTERIOR BRUCE BABBITT, Secretary
}

\author{
U.S. GEOLOGICAL SURVEY \\ Gordon P. Eaton, Director
}

Any use of trade, product, or firm name in this report is for identification purposes only and does not constitute endorsement by the U.S.

Geological Survey.

For additional information write to:

District Chief

U.S. Geological Survey

810 Broadway, Suite 500

Nashville, Tennessee 37203
Copies of this report may be purchased from:

U.S. Geological Survey

Earth Science Information Center

Open-File Reports Section

Box 25286, MS 517

Denver Federal Center

Denver, Colorado 80225 


\section{CONTENTS}

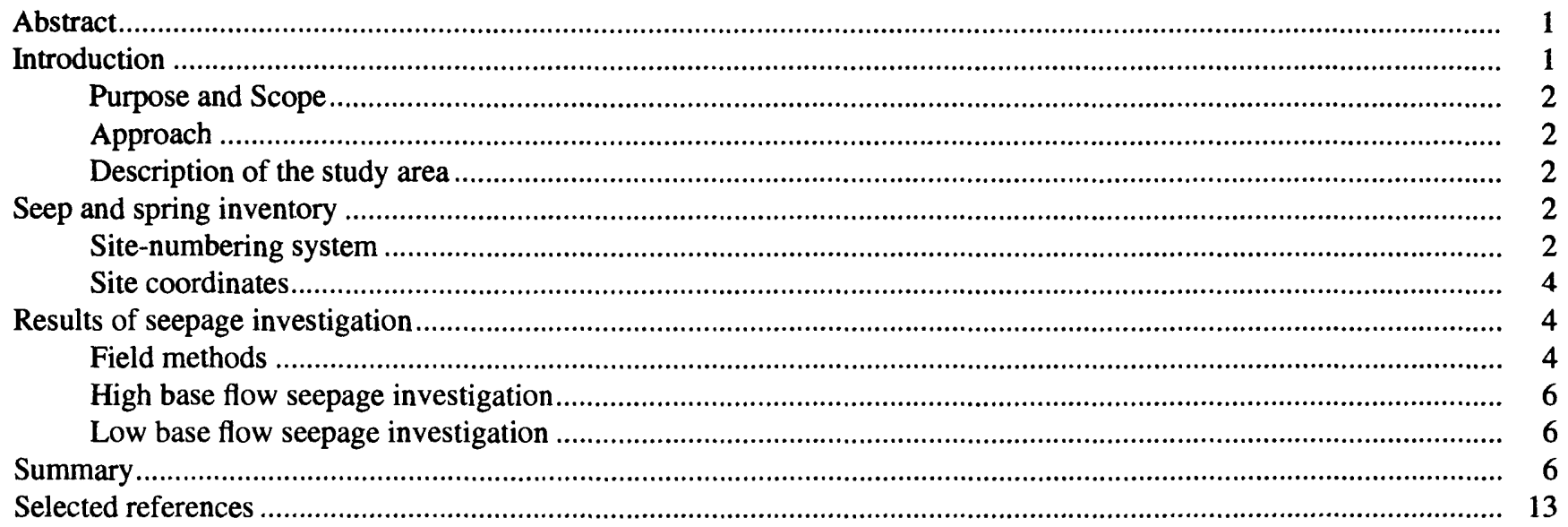

\section{PLATES}

(Plates are in pocket)

1. Map showing location of seeps, springs, and stream-measurement sites at Oak Ridge National Laboratory, Oak Ridge, Tennessee, for the high base flow seepage investigation April 29 through May 3, 1993 and May 7 through May 10, 1993

2. Map showing location of seeps, springs, and stream-measurement sites at Oak Ridge National Laboratory, Oak Ridge, Tennessee, for the low base flow seepage investigation August 7 through August 10, 1993

\section{FIGURES}

1. Map showing study area at Oak Ridge National Laboratory, Oak Ridge, Tennessee

2. Graph showing daily mean discharge at Whiteoak Creek near Melton Hill, Tennessee, and daily rainfall at Whiteoak Creek near Melton Valley Road, at Oak Ridge, Tennessee, from March 1 through September 1, 1993.

3. Map of sampling area 19 showing location of sites for intensive sampling on Center Seven Tributary to Melton Branch, April 30, 1993

4. Map of sampling area 20 showing location of sites for intensive sampling on Bearden Creek, May 8, 1993 


\section{TABLES}

1. Size of sub-basin sampling areas in the Oak Ridge National Laboratory seepage investigation ...

2. Coordinates for seeps, springs, and stream-measurement sites at Oak Ridge National Laboratory

3. Discharge and water-quality data for the high base flow seepage investigation at

Oak Ridge National Laboratory, April 29 through May 10, 1993

4. Statistical summary of discharge and water-quality data for the high base flow seepage investigation at Oak Ridge National Laboratory.

5. Water-quality data for Center Seven Tributary to Melton Branch at Oak Ridge National Laboratory on April 30, 1993.

6. Statistical summary of water-quality data for Center Seven Tributary to Melton Branch at Oak Ridge National Laboratory on April 30, 1993

7. Water-quality data for Bearden Creek at Oak Ridge National Laboratory on May 8, 1993

8. Statistical summary of water-quality data for Bearden Creek at Oak Ridge National Laboratory on May 8, 1993.

9. Discharge and water-quality data for the low base flow seepage investigation at Oak Ridge

National Laboratory, September 8-10, 1993

10. Statistical summary of discharge and water-quality data for the low base flow seepage investigation at Oak Ridge National Laboratory.

\section{CONVERSION FACTORS AND VERTICAL DATUM}

\begin{tabular}{rll}
\hline Muitiply & By & To obtain \\
\hline foot $(\mathrm{ft})$ & 0.3048 & \\
cubic foot per second $\left(\mathrm{ft}^{3} / \mathrm{s}\right)$ & 0.02832 & meter \\
acre & 0.4047 & cubic meters per second \\
mile $(\mathrm{mi})$ & 1.609 & square hecometer \\
square mile $\left(\mathrm{mi}^{2}\right)$ & 2.590 & kilometer \\
\hline
\end{tabular}

Temperature in degrees Celsius $\left({ }^{\circ} \mathrm{C}\right)$ can be converted to degrees Fahrenheit $\left({ }^{\circ} \mathrm{F}\right)$ as follows: ${ }^{\circ} \mathrm{F}=1.8 \mathrm{x}{ }^{\circ} \mathrm{C}+32$

Sea level: In this report "sea level" refers to the National Geodetic Vertical Datum of 1929--a geodetic datum derived from a general adjustment of the first-order level nets of the United States and Canada, formerly called Sea Level Datum of 1929.

Water-quality unit:

$\mu \mathrm{S} / \mathrm{cm} \quad$ microsiemens per centimeter at 25 degrees Celsius 


\section{A SEEPAGE INVESTIGATION OF AN AREA AT AND NEAR OAK RIDGE NATIONAL LABORATORY, OAK RIDGE, TENNESSEE, MARCH THROUGH AUGUST 1993}

\section{BYGREGORY C. JOHNSON}

\section{ABSTRACT}

A seepage investigation was conducted of an area surrounding the Oak Ridge National Laboratory for the period of March through August 1993. The project was divided into three phases: a reconnaissance to inventory and map seeps, springs, and stream-measurement sites; a high base flow seepage investigation; and a low base flow seepage investigation.

The reconnaissance consisted of following each tributary to its source to inventory each site where water was issuing from the ground.

Stream-measurement sites were located along stream reaches at approximately 500 -foot intervals. A total of 822 sites were identified. A global positioning system was used to locate 483 sites to within 3- to 5-meter accuracy.

The high base flow seepage investigation was conducted from April 29 through May 3, 1993, and from May 7 through May 10, 1993. Measurements were made at 604 of the 822 sites identified in the reconnaissance. Flow rates ranged from 0 to 2.15 cubic feet per second $\left(\mathrm{ft}^{3} / \mathrm{s}\right)$ for the streams, from 0 to $0.07 \mathrm{ft}^{3} / \mathrm{s}$ for the seeps, and from 0 to $0.55 \mathrm{ft}^{3} / \mathrm{s}$ for the springs. Values of $\mathrm{pH}$ ranged from 5.1 to 8.5 for the streams, from 4.8 to 8.3 for the seeps, and from 5.9 to 8.0 for the springs. Specific conductance ranged from 21 to 1,004 microsiemens per centimeter $(\mu \mathrm{S} / \mathrm{cm})$ for the streams, 14 to $687 \mu \mathrm{S} / \mathrm{cm}$ for the seeps, and from 28 to $589 \mu \mathrm{S} / \mathrm{cm}$ for the springs.
Two stream reaches, one on Center Seven Tributary to Melton Branch and one on Bearden Creek, were intensively sampled in conjunction with the high base flow seepage investigation. On April 30, 1993 at Center Seven Tributary to Melton $\mathrm{Branch}$, the $\mathrm{pH}$ ranged from 6.4 to 7.6 ; specific conductance, from 121 to $265 \mu \mathrm{S} / \mathrm{cm}$; and temperature, from 11.5 to $20.0^{\circ} \mathrm{C}$. On May 8, 1993 at Bearden Creek, the $\mathrm{pH}$ ranged from 7.7 to 8.0; specific conductance, from 371 to $458 \mu \mathrm{S} / \mathrm{cm}$; and temperature, from 14.0 to $22.0^{\circ} \mathrm{C}$.

The low base flow investigation was conducted from August 8 through August 10, 1993. The seeps and springs that were flowing during the high base flow seepage investigation were revisited. Twenty-seven of the 147 seeps visited were flowing; discharge ranged from 0 to $0.07 \mathrm{ft}^{3} / \mathrm{s} ; \mathrm{pH}$, from 6.7 to 8.1 ; specific conductance, from 59 to $516 \mu \mathrm{S} / \mathrm{cm}$; and temperature, from 14.0 to $23.0^{\circ} \mathrm{C}$. Forty of the 61 springs visited were flowing; discharge ranged from 0 to $0.34 \mathrm{ft}^{3} / \mathrm{s} ; \mathrm{pH}$, from 6.6 to 8.0 ; specific conductance, from 87 to $572 \mu \mathrm{S} / \mathrm{cm}$; and temperature, from 13.0 to $20.5^{\circ} \mathrm{C}$.

\section{INTRODUCTION}

The U.S. Geological Survey (USGS), in cooperation with the U.S. Department of Energy, conducted a seepage investigation on 4,328 acres surrounding the Oak Ridge National Laboratory (ORNL). The information from this project will aid the ORNL 
Environmental Restoration Program, Groundwater Operable Units Remedial Investigations Project to develop a better understanding of ground-water and surface-water interaction on the Oak Ridge Reservation.

\section{Purpose and Scope}

This report describes the results of a seepage investigation conducted at ORNL from March through August 1993. The report includes a map showing site locations, and tables showing discharge, $\mathrm{pH}$, specific conductance, temperature, and the site coordinates.

\section{Approach}

The study involved three phases of activity: (1) a reconnaissance to inventory and map seeps, springs, and stream-measurement sites (2) the measurement of discharge and water-quality characteristics during high base flow conditions, and (3) the measurement of discharge and water-quality characteristics under low base flow conditions.

\section{Description of the Study Area}

The Oak Ridge Reservation (ORR) is located in East Tennessee. It is in the Valley and Ridge Province at a point where the Tennessee salient of the Appalachian foreland fold-thrust belt is narrowest and most convex (Hatcher and others, 1992). Ordovician and Cambrian rocks that underlie the Valley and Ridge Province are predominantly carbonate, siltstone, shale, and some sandstone.

The 58,000-acre ORR is bounded on the northeast, southeast, and southwest by the Clinch River, and on the northwest by Blackoak Ridge (McMaster, 1967). The three major facilities in the area are $X-10$, the Oak Ridge National Laboratory, a research and development center; Y-12, a research, development, and production center; and K-25, the Gaseous Diffusion Plant (ORGDP), a production center that was closed in 1986.

The ORNL seepage investigation study area is bounded by Copper Ridge on the southeast, Clinch River on the southwest, Bearden Creek watershed on the northeast, and an arbitrary boundary along the electric power lines on the northwest (fig. 1). Certain areas within the study area were not sampled due to safety or security concerns. Eighteen sampling areas encompassing a total area of 4,328 acres were included in the study (table 1 ).

\section{SEEP AND SPRING INVENTORY}

A reconnaissance was conducted from March 28 to April 20, 1993 to inventory and map the location of seeps, springs, and stream-measurement sites. For each of the 18 sampling areas, all streams and tributaries were followed to their source or to an area boundary. A total of 822 sites were staked and assigned unique identification numbers. The inventory consists of 279 seeps, 68 springs, and 475 stream-measurement sites (plate 1).

\section{Site-Numbering System}

Each site was assigned a unique identification number in which the sampling area number was used for the first two digits, and an upstream order number from 001 to 999 for the last three digits (table 2, located in back of report). This approach assigns the lowest number to the most downstream site in the

Table 1. Size of sub-basin sampling areas in the Oak Ridge National Laboratory seepage investigation

\begin{tabular}{|c|c|c|}
\hline $\begin{array}{l}\text { Sampling } \\
\text { area }\end{array}$ & $\begin{array}{c}\text { Area, } \\
\text { in acres }\end{array}$ & $\begin{array}{c}\text { Area, in } \\
\text { square miles }\end{array}$ \\
\hline 1 & 168.1 & 0.26 \\
\hline 2 & 609.0 & .95 \\
\hline 3 & 413.5 & .65 \\
\hline 4 & 112.6 & .18 \\
\hline 5 & 345.7 & .54 \\
\hline 6 & 263.4 & .41 \\
\hline 7 & 207.8 & .32 \\
\hline 8 & 207.1 & .32 \\
\hline 9 & 199.7 & .31 \\
\hline 10 & 237.6 & .37 \\
\hline 11 & 167.2 & .26 \\
\hline 12 & 205.7 & .32 \\
\hline 13 & 205.7 & .32 \\
\hline 14 & 135.0 & .21 \\
\hline 15 & 46.5 & .07 \\
\hline 16 & 281.0 & .44 \\
\hline 17 & 280.5 & .44 \\
\hline 18 & 242.4 & .38 \\
\hline Total & $4,328.5$ & 6.75 \\
\hline
\end{tabular}




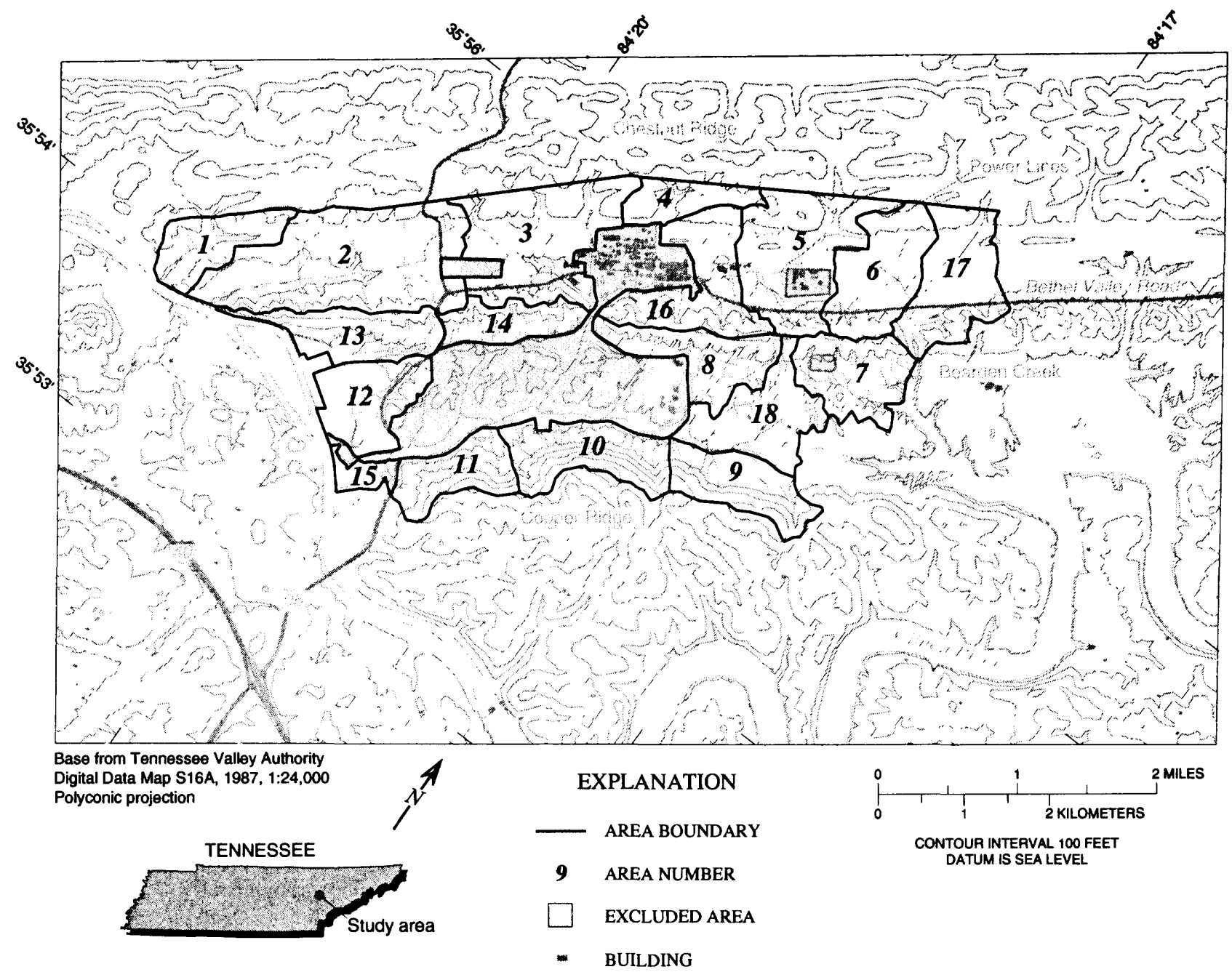

Figure 1. Study area at Oak Ridge National Laboratory, Oak Ridge, Tennessee. 
basin, and increasingly larger numbers to sites upstream. When a tributary is reached, site numbers increase along the tributary upstream to its source, and numbering resumes along the main channel. Included within sampling areas 18 and 6 are two intensively sampled stream reaches identified as areas 19 and 20 (locations shown in figs. 3 and 4, respectively).

The sites were classified as either a seep (SE), spring (SP), or stream-measurement site (Q) (table 3, located at back of report). A seep was defined as a discharge of water from the ground without a distinguishable point outlet and generally at a low flow rate. A spring was defined as a discharge of water at a defined outlet or where water was upwelling. The presence of watercress usually was an indicator of a spring. Stream-measurement sites were located about every 500 feet along each channel with flowing water, and at the confluence of tributaries with flow. In some cases, every seep could not be defined; therefore, some sites are noted as seepage reaches (SE/R) or seepage areas (SE/A). A seepage reach is where multiple seeps occur along a section of the channel reach, and a seepage area is where multiple undifferentiated seeps occur in a defined area.

\section{Site Coordinates}

A global positioning system (GPS) was used to determine the coordinates of 483 sites to within 3- to 5-meter accuracy. The remaining sites were mapped by measuring their distances from the GPS points or other control points, plotting locations on field maps, and digitizing these points. Locations of 894 sites, consisting of the seepage investigation sites and sites along the intensively sampled stream reaches, are reported in ORNL grid coordinates and State plane coordinates (table 2, located at back of report).

\section{RESULTS OF SEEPAGE INVESTIGATION}

Two seepage investigations were conducted, one during high base flow and one during low base flow. A waiting period of 72 hours after rainfall events was used to minimize the possible influence of ephemeral streams and springs (Mulholland, 1991). Measurements in each sampling area were completed in 1 day to minimize variations in flow rates. During the high base flow seepage investigation, discharge and water-quality measurements were made at each site with flowing water. For the low base flow seepage investigation, only the seeps and springs that were flowing during the high base flow canvass were revisited.

\section{Field Methods}

Four methods were used to measure discharge: (1) volumetric measurements, (2) current-meter measurements, (3) surface velocity measurements, and (4) estimations.

Volumetric measurements are typically the most accurate method for quantifying discharge during lowflow conditions. For this study, a volume of water was collected during a timed interval for four iterations, and the discharge was calculated as the average of the four measurements.

Pygmy-type current meters were used where practical. Using standard USGS current-meter discharge-measurement procedures, the accuracy of measurements range from about 5 percent error on some of the larger streams to greater than 8 percent error on some sites with low discharge.

The third type of discharge measurement involved the use of floats. The cross-sectional area of a stream was measured, and the average time for a float to travel a known distance for four iterations was determined. Discharge was then calculated and reported to one one-hundredth of a cubic foot per second.

The final, and least accurate, method was to estimate the discharge. This method was used in cases where the discharge was very small, and no other method was practical.

A notation was made to indicate sites where flow was visible, but less than the minimum reportable discharge. At sites where the flow was less than five one-thousandths of a cubic foot per second or $0.005 \mathrm{ft}^{3} / \mathrm{s}$, the flow was reported as zero. For flows greater than five one-thousandths of a cubic foot per second, the discharge was rounded to the nearest hundredth of a cubic foot per second.

Conductance and $\mathrm{pH}$ meters were used to collect specific conductance, temperature, and $\mathrm{pH}$. The meters were calibrated every morning before use, and again in the field if questionable values occurred.

Discharge at the Whiteoak Creek near Melton Hill, Tennessee, stream gage (03536320) and rainfall at Whiteoak Creek near Melton Valley Road (03536550) were used as indicators of the hydrologic conditions for the study area (fig. 2). During the 


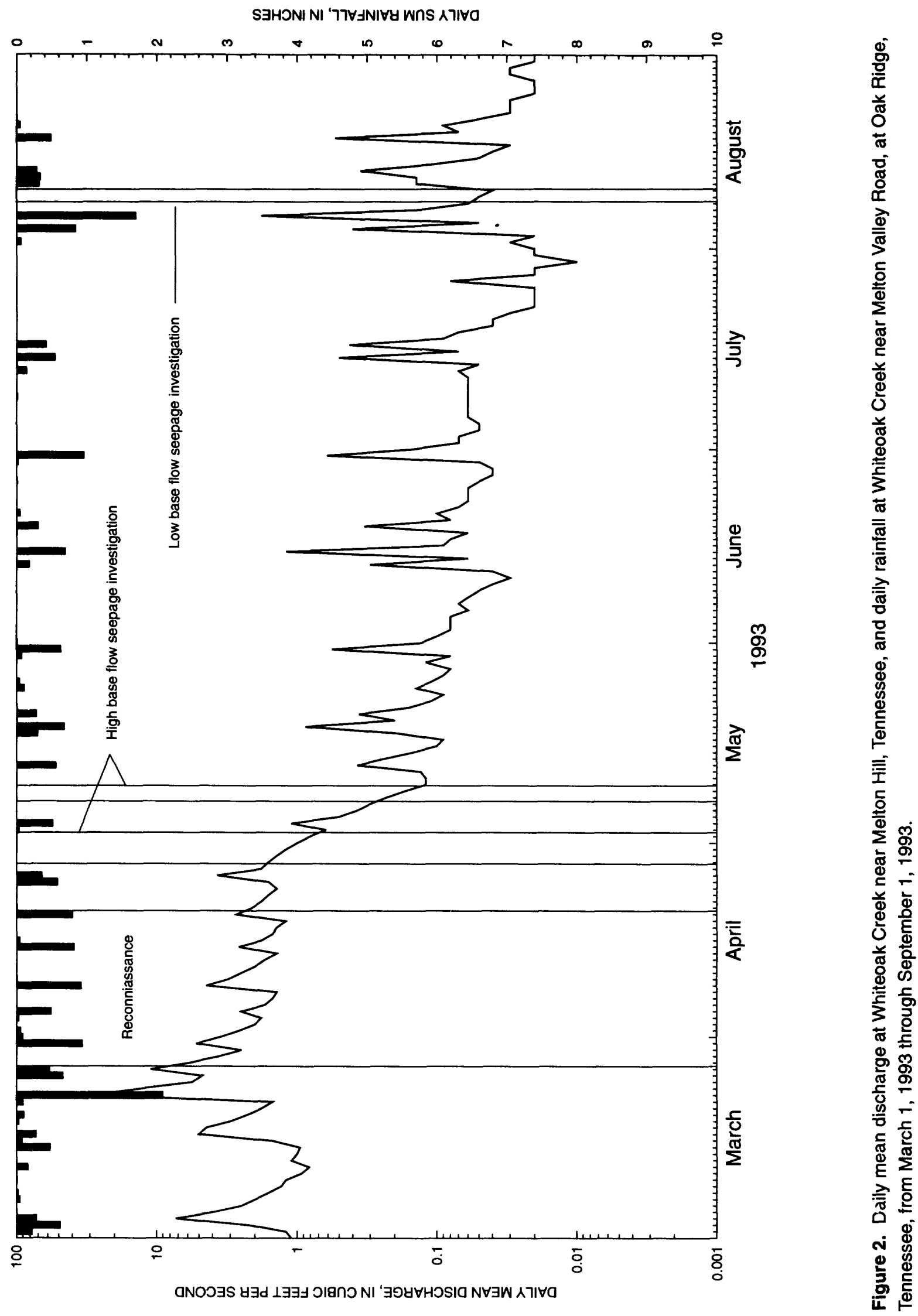


reconnaissance, March 28 through April 20, 1993, daily mean discharge at Whiteoak Creek ranged from 1.2 to $5.8 \mathrm{ft}^{3} / \mathrm{s}$. The daily mean discharge ranged from 0.12 to $1.4 \mathrm{ft}^{3} / \mathrm{s}$ during the high base flow seepage investigation and from 0.04 to $0.06 \mathrm{ft}^{3} / \mathrm{s}$ during the low base flow seepage investigation.

\section{High Base Flow Seepage Investigation}

The high base flow seepage investigation was conducted from April 29 through May 3, 1993, and from May 7 through May 10, 1993. All 822 sites identified during the reconnaissance were revisited. Discharge and water-quality measurements were made at 604 sites with flowing water (plate 1 , table 3 , located at back of report). At 236 of the 604 sites with flowing water, the flow was reported as zero with a notation that the flow was less than the minimum reportable discharge $\left(0.005 \mathrm{ft}^{3} / \mathrm{s}\right)$. Flow rates ranged from 0 to $2.15 \mathrm{ft}^{3} / \mathrm{s}$ for the streams, from 0 to $0.07 \mathrm{ft}^{3} / \mathrm{s}$ for the seeps, and from 0 to $0.55 \mathrm{ft}^{3} / \mathrm{s}$ for the springs (table 4 ). Values for $\mathrm{pH}$ ranged from 5.1 to 8.5 for the streams, from 4.8 to 8.3 for the seeps, and from 5.9 to 8.0 for the springs. Specific conductance ranged from 21 to 1,004 microsiemens per centimeter $(\mu \mathrm{S} / \mathrm{cm})$ for the streams, 14 to $687 \mu \mathrm{S} / \mathrm{cm}$ for the seeps, and from 28 to $589 \mu \mathrm{S} / \mathrm{cm}$ for the springs. Temperature ranged from 9.5 to $24.0^{\circ} \mathrm{C}$ for the streams, 11.0 to $21.0^{\circ} \mathrm{C}$ for the seeps, and 11.5 to $18.5^{\circ} \mathrm{C}$ for the springs.

In conjunction with the high base flow seepage investigation, two stream reaches were intensively sampled for $\mathrm{pH}$, specific conductance, and temperature. The data were collected at points where changes in flow contribution, geology, or channel morphology occurred. Center Seven Tributary to Melton Branch was evaluated on April 30, 1993, and Bearden Creek was evaluated on May 8, 1993 (plate 1). Coordinates for these points are listed in table 2.

Center Seven Tributary to Melton Branch

(fig. 3) was selected for sampling due to the number of reaches where the stream flowed into the ground through macropores in the stream bed (swallets) and then reemerged downstream. Center Seven Tributary is also characterized by having braided channel reaches. The site numbering system for this part of the study (table 5, and fig. 3) used 19 for the first two digits and 001 through 051 for the last three digits. The sampling on Center Seven Tributary to Melton Branch started at site 18070 (plate 1). The temperature ranged from 11.5 to $20.0^{\circ} \mathrm{C}$; specific conductance, from 121 to $265 \mu \mathrm{S} / \mathrm{cm}$; and $\mathrm{pH}$, from 6.4 to 7.6 (table 6).

A section of Bearden Creek (fig. 4) was also selected for intensive sampling based on the occurrence of many limestone and shale outcroppings in the stream bed. Water-quality measurements were taken adjacent to these outcroppings. The sampling on Bearden Creek started at the triple channel junction where sites 06020 and 06055 are located (plate 1). The east and middle branches of the channels were flowing, and temperature, specific conductance, and $\mathrm{pH}$ were measured at points along these channels (table 7). The site numbering system for this study uses 20 for the first two digits and 001 through 019 for the last three digits. The temperature ranged from 14.0 to $22.0^{\circ} \mathrm{C}$; specific conductance, from 371 to $458 \mu \mathrm{S} / \mathrm{cm}$; and $\mathrm{pH}$, from 7.7 to 8.0 (table 8 ).

\section{Low Base Flow Seepage Investigation}

The seeps and springs that were flowing during the high base flow seepage investigation were revisited during the low base flow seepage investigation (plate 2, table 9, located at back of report). Stream sites were not revisited. Two additional seeps, 03718 and $08280 \mathrm{a}$, and two springs, 02356 and $05070 \mathrm{a}$, not identified during the reconnaissance, were added during the low base flow seepage phase of the study. At 20 spring sites and 19 seep sites, the flow rate was reported as zero with a notation that the flow was less than the minimum reportable discharge $\left(0.005 \mathrm{ft}^{3} / \mathrm{s}\right)$. Twenty-seven of the 147 seeps visited were flowing, and had discharge ranging from 0 to $0.07 \mathrm{ft}^{3} / \mathrm{s} ; \mathrm{pH}$, from 6.7 to 8.1 ; specific conductance, from 59 to $516 \mu \mathrm{S} / \mathrm{cm}$; and temperature, from 14.0 to $23.0^{\circ} \mathrm{C}$. Forty of the 61 springs revisited were flowing, and had discharge ranging from 0 to $0.34 \mathrm{ft}^{3} / \mathrm{s} ; \mathrm{pH}$, from 6.6 to 8.0; specific conductance, from 87 to $572 \mu \mathrm{S} / \mathrm{cm}$; and temperature, from 13.0 to $20.5^{\circ} \mathrm{C}$ (table 10).

\section{SUMMARY}

A seepage investigation was conducted in the area surrounding Oak Ridge National Laboratory for the period of March through August 1993. The study was performed in three phases: a reconnaissance and mapping of the sites, a high base flow seepage investigation, and a low base flow seepage investigation.

The reconnaissance was conducted from March 28 through April 20,1993, to identify and map seeps, 
Table 4. Statistical summary of discharge and water-quality data for the high base flow seepage investigation at Oak Ridge National Laboratory

[ft ${ }^{3} / \mathrm{s}$, cubic feet per second; $\mu \mathrm{S} / \mathrm{cm}$, microsiemens per centimeter at $25^{\circ} \mathrm{C} ;{ }^{\circ} \mathrm{C}$, degrees Celsius; --, not meaningful]

\begin{tabular}{|c|c|c|c|c|}
\hline \multicolumn{5}{|c|}{$\begin{array}{l}\text { Stream-measurement sites } \\
\text { (exciuding } 69 \text { dry sites, and } 1 \text { unmeasured site) }\end{array}$} \\
\hline Statistic & $\begin{array}{l}\text { Discharge } \\
\left(\left(t^{3} / 8\right)\right.\end{array}$ & pH & $\begin{array}{c}\text { Specific } \\
\text { conductance } \\
(\mu \mathrm{S} / \mathrm{cm})\end{array}$ & $\begin{array}{l}\text { Temperature } \\
\left({ }^{\circ} \mathrm{C}\right)\end{array}$ \\
\hline Number of measurements & 404 & 400 & 404 & 404 \\
\hline Number of missed measurements & 1 & 5 & 1 & 1 \\
\hline Minimum value & 0 & 5.1 & 21 & 9.5 \\
\hline Maximum value & 2.15 & 8.5 & 1,004 & 24.0 \\
\hline Mean & .12 & -- & 244 & 15.2 \\
\hline Standard deviation & .27 & .6 & 135 & 2.5 \\
\hline 25th percentile & 0 & 7.2 & 139 & 13.5 \\
\hline Median & .02 & 7.4 & 225 & 15.2 \\
\hline 75th percentile & .07 & 7.8 & 333 & 16.5 \\
\hline \multicolumn{5}{|c|}{$\begin{array}{c}\text { Seep sites } \\
\text { (exciuding } 137 \text { dry sites, and } 2 \text { unmeasured sites) }\end{array}$} \\
\hline Statistic & $\begin{array}{c}\text { Discharge } \\
\left(\mathrm{ft}^{3} / \mathrm{s}\right)\end{array}$ & pH & $\begin{array}{c}\text { Specific } \\
\text { conductance } \\
(\mu \mathrm{S} / \mathrm{cm})\end{array}$ & $\begin{array}{l}\text { Temperature } \\
\left({ }^{\circ} \mathrm{C}\right)\end{array}$ \\
\hline Number of measurements & 136 & 138 & 138 & 139 \\
\hline Number of missed measurements & 4 & 2 & 2 & 1 \\
\hline Minimum value & 0 & 4.8 & 14 & 11.0 \\
\hline Maximum value & .07 & 8.3 & 687 & 21.0 \\
\hline Mean & 0 & -- & 248 & 14.5 \\
\hline Standard deviation & .01 & .7 & 185 & 2.0 \\
\hline 25th percentile & 0 & 6.5 & 90 & 13.0 \\
\hline Median & 0 & 6.9 & 186 & 14.0 \\
\hline 75th percentile & 0 & 7.4 & 370 & 15.0 \\
\hline \multicolumn{5}{|c|}{$\begin{array}{c}\text { Spring sites } \\
\text { (exciuding } 8 \text { dry sites, and } 1 \text { unmeasured site) }\end{array}$} \\
\hline Statistic & $\begin{array}{c}\text { Discharge } \\
\left(\mathrm{ft}^{3} / \mathrm{s}\right)\end{array}$ & pH & $\begin{array}{c}\text { Specific } \\
\text { conductance } \\
(\mu S / \mathrm{cm})\end{array}$ & $\begin{array}{l}\text { Temperature } \\
\left({ }^{\circ} \mathrm{C}\right)\end{array}$ \\
\hline Number of measurements & 58 & 59 & 59 & 59 \\
\hline Number of missed measurements & 1 & 0 & 0 & 0 \\
\hline Minimum value & 0 & 5.9 & 28 & 11.5 \\
\hline Maximum value & .55 & 8.0 & 589 & 18.5 \\
\hline Mean & .05 & -- & 272 & 13.5 \\
\hline Standard deviation & .09 & .4 & 136 & 1.3 \\
\hline 25th percentile & .01 & 6.8 & 193 & 12.5 \\
\hline Median & .03 & 7.1 & 255 & 13.5 \\
\hline 75th percentile & .05 & 7.3 & 351 & 14.0 \\
\hline
\end{tabular}



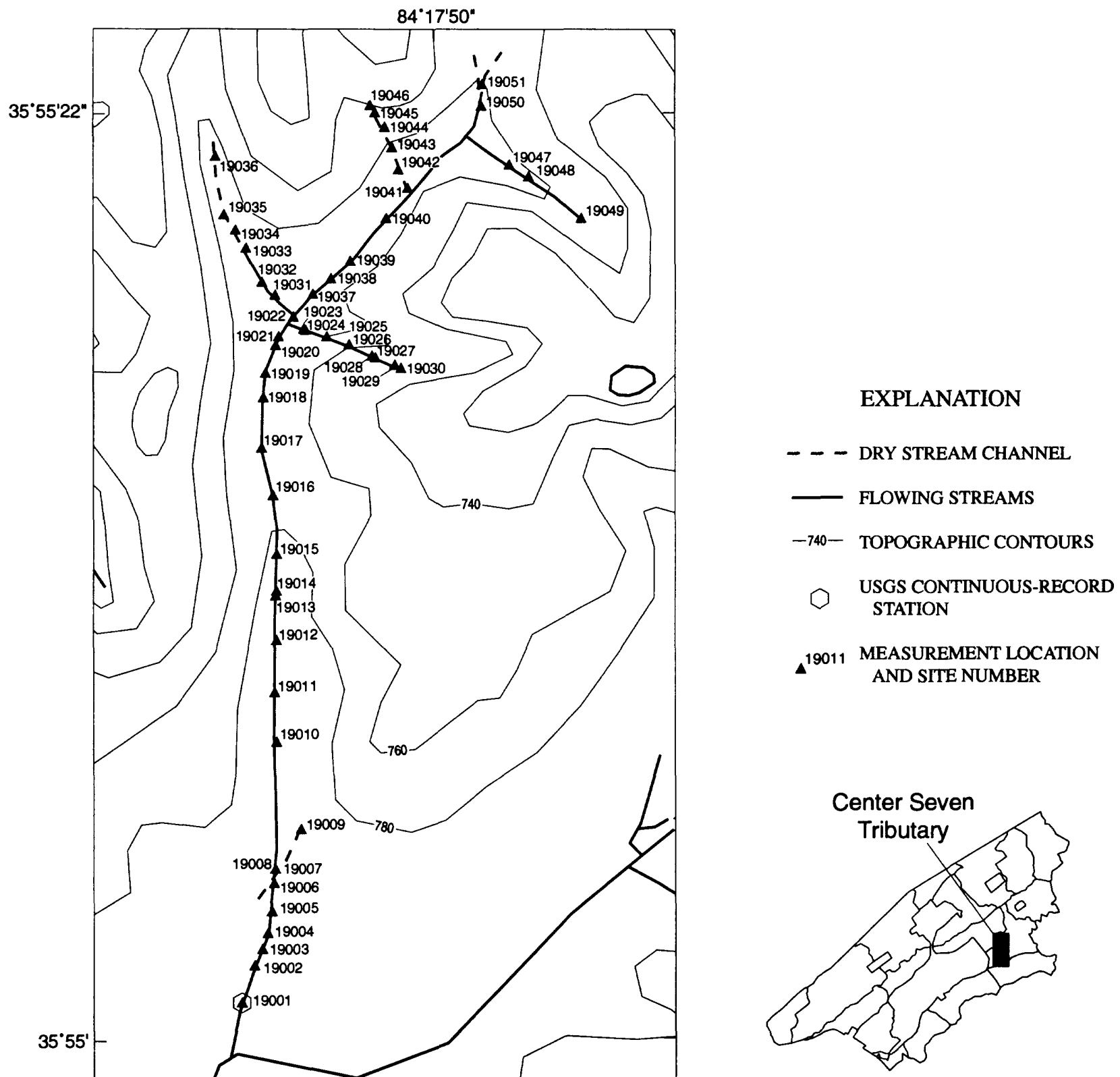

Figure 3. Sampling area 19 showing location of sites for intensive sampling on Center Seven Tributary to Melton Branch, April 30, 1993. 
Table 5. Water-quality data for Center Seven Tributary to Melton Branch at Oak Ridge National Laboratory on April 30, 1993

$\left[{ }^{\circ} \mathrm{C}\right.$, degrees Celsius; $\mu \mathrm{S} / \mathrm{cm}$, microsiemens per centimeter at $25^{\circ} \mathrm{C}$; $\mathrm{ft}$, feet; a, multiple measurements at different times during the day]

\begin{tabular}{|c|c|c|c|}
\hline Site number & $\begin{array}{l}\text { Temperature } \\
\left({ }^{\circ} \mathrm{C}\right)\end{array}$ & $\begin{array}{c}\text { Specific } \\
\text { conductance } \\
(\mu \mathrm{S} / \mathrm{cm})\end{array}$ & pH \\
\hline 19001 & 12.5 & 238 & 7.3 \\
\hline 19002 & 15.5 & 239 & 7.2 \\
\hline 19003 & 15.5 & 168 & 7.2 \\
\hline 19004 & 12.5 & 244 & 7.3 \\
\hline 19005 & 12.5 & 248 & 7.4 \\
\hline 19006 & 12.5 & 248 & 7.4 \\
\hline 19007 & 12.5 & 255 & 7.5 \\
\hline 19008 & 13.0 & 198 & 7.0 \\
\hline 19009 & 13.0 & 180 & 6.7 \\
\hline 19010 & 13.0 & 260 & 7.3 \\
\hline 19011 & 13.5 & 261 & 7.4 \\
\hline \multirow[t]{3}{*}{$19012 a$} & 13.5 & 257 & \\
\hline & 15.5 & 260 & 7.5 \\
\hline & 16.0 & 260 & 7.6 \\
\hline 19013 & 16.0 & 259 & 7.4 \\
\hline 19014 & 13.5 & 253 & 7.3 \\
\hline 19015 & 16.5 & 243 & 7.4 \\
\hline 19016 & 16.5 & 226 & 7.5 \\
\hline 19017 & 16.5 & 226 & 7.6 \\
\hline 19018 & 16.5 & 212 & 7.3 \\
\hline 19019 & 16.5 & 202 & 7.3 \\
\hline 19020 & 16.5 & 158 & 7.4 \\
\hline 19021 & 16.5 & 175 & 7.4 \\
\hline 19022 & 16.0 & 193 & 7.4 \\
\hline 19023 & 14.0 & 180 & 6.7 \\
\hline 19024 & 16.0 & 186 & 7.4 \\
\hline 19025 & 17.0 & 198 & 7.1 \\
\hline 19026 & 20.0 & 189 & 7.3 \\
\hline 19027 & 19.5 & 194 & 7.1 \\
\hline 19028 & 16.5 & 157 & 7.4 \\
\hline 19029 & 19.5 & 155 & 6.8 \\
\hline 19030 & 16.0 & 188 & 6.4 \\
\hline 19031 & 14.0 & 173 & 7.0 \\
\hline 19032 & 13.5 & 134 & 7.0 \\
\hline 19033 & 14.5 & 185 & 7.0 \\
\hline 19034 & 13.5 & 177 & 7.0 \\
\hline 19035 & 14.0 & 179 & 7.0 \\
\hline 19036 & 13.0 & 183 & 6.5 \\
\hline 19037 & 17.0 & 189 & 7.6 \\
\hline 19038 & 16.5 & 183 & 7.6 \\
\hline 19039 & 16.5 & 187 & 7.6 \\
\hline 19040 & 16.5 & 188 & 7.4 \\
\hline 19041 & 16.5 & 174 & 7.2 \\
\hline 19042 & 17.0 & 197 & 7.2 \\
\hline 19043 & 16.5 & 206 & 7.3 \\
\hline 19044 & 16.5 & 199 & 7.2 \\
\hline 19045 & 17.0 & 257 & 7.5 \\
\hline 19046 & 16.0 & 176 & 7.2 \\
\hline 19047 & 15.0 & 235 & 7.4 \\
\hline 19048 & 14.0 & 263 & 7.4 \\
\hline 19049 & 11.5 & 265 & 7.6 \\
\hline 19050 & 15.5 & 121 & 6.9 \\
\hline 19051 & 14.0 & 122 & 6.4 \\
\hline
\end{tabular}


Table 6. Statistical summary of water-quality data for Center Seven Tributary to Melton Branch at Oak Ridge National Laboratory on April 30, 1993

$\left[\mu \mathrm{S} / \mathrm{cm}\right.$, microsiemens per centimeter at $25^{\circ} \mathrm{C} ;{ }^{\circ} \mathrm{C}$, degrees Celsius;,-- not meaningful]

\begin{tabular}{lccc}
\hline \multicolumn{1}{c}{ Statistic } & $\mathbf{p H}$ & $\begin{array}{c}\text { Specific } \\
\text { (onductance } \\
(\mu \text { S/cm) })\end{array}$ & $\begin{array}{c}\text { Temperature } \\
\left({ }^{\circ} \mathbf{C}\right)\end{array}$ \\
\hline Number of measurements & 52 & 53 & 53 \\
Minimum value & 6.4 & 121 & 11.5 \\
Maximum value & 7.6 & 265 & 20.0 \\
Mean & - & 206 & 15.2 \\
Standard deviation & 0.3 & 39 & 1.9 \\
25th percentile & 7.0 & 179 & 13.5 \\
Median & 7.3 & 197 & 15.5 \\
75th percentile & 7.4 & 244 & 16.5 \\
\hline
\end{tabular}

Table 7. Water-quality data for Bearden Creek at Oak Ridge National Laboratory on May 8, 1993

$\left[{ }^{\circ} \mathrm{C}\right.$, degree Celsius; $\mu \mathrm{S} / \mathrm{cm}$, microsiemens per centimeter at $25^{\circ} \mathrm{C}$; a, site measured twice at different times during the day]

\begin{tabular}{lccc}
\hline $\begin{array}{c}\text { Site } \\
\text { number }\end{array}$ & $\begin{array}{c}\text { Temperature } \\
\left({ }^{\circ} \mathbf{C}\right)\end{array}$ & $\begin{array}{c}\text { Specific } \\
\text { Conductance } \\
(\mu \text { S/cm })\end{array}$ & pH \\
\hline 20001 & 14.0 & 405 & 7.9 \\
$20001 \mathrm{a}$ & 18.5 & 393 & 8.0 \\
20002 & 14.5 & 379 & 7.9 \\
20003 & 14.0 & 425 & 7.8 \\
20004 & 14.0 & 450 & 7.7 \\
20005 & 14.5 & 456 & 7.8 \\
20006 & 14.5 & 451 & 7.8 \\
20007 & 14.5 & 452 & 7.8 \\
20008 & 14.5 & 457 & 7.8 \\
20009 & 15.5 & 458 & 7.8 \\
20010 & 18.5 & 379 & 7.9 \\
20011 & 19.0 & 380 & 7.9 \\
20012 & 19.0 & 380 & 7.8 \\
20013 & 19.0 & 380 & 7.7 \\
20014 & 19.0 & 380 & 8.8 \\
20015 & 19.5 & 376 & 8.0 \\
20016 & 20.5 & 371 & 7.9 \\
20017 & 21.0 & 371 & 7.7 \\
20018 & 21.5 & 372 & \\
\hline
\end{tabular}




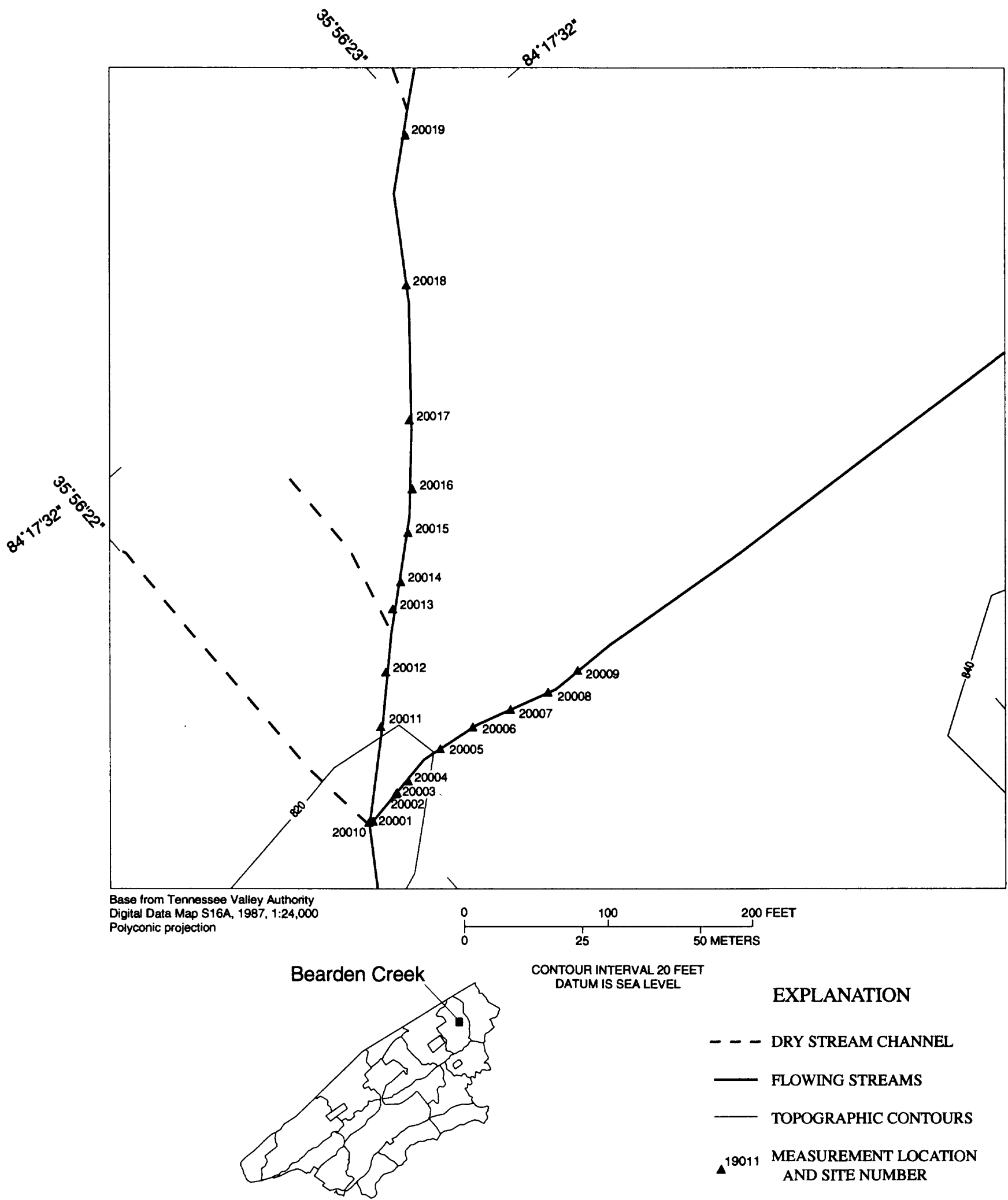

Figure 4. Sampling area 20 showing location of sites for intensive sampling on Bearden Creek, May 8, 1993. 
Table 8. Statistical summary of water-quality data for Bearden Creek at Oak Ridge National Laboratory on May 8,1993 $\left[\mu \mathrm{S} / \mathrm{cm}\right.$, microsiemens per centimeter at $25^{\circ} \mathrm{C} ;{ }^{\circ} \mathrm{C}$, degrees Celsius; --, not meaningful]

\begin{tabular}{lccc}
\hline \multicolumn{1}{c}{ Statistic } & pH & $\begin{array}{c}\text { Specific } \\
\text { conductance } \\
(\mu \mathbf{S} / \mathbf{c m})\end{array}$ & $\begin{array}{c}\text { Temperature } \\
\text { ( } \mathbf{C})\end{array}$ \\
\hline Number of measurements & 20 & 20 & 20 \\
Minimum value & 7.7 & 371 & 14.0 \\
Maximum value & 8.0 & 458 & 22.0 \\
Mean & - & 406 & 17.6 \\
Standard deviation & 0.1 & 36 & 2.8 \\
25th percentile & 7.8 & 376 & 14.5 \\
Median & 7.8 & 380 & 18.5 \\
75th percentile & 7.9 & 450 & 19.0 \\
\hline
\end{tabular}

Table 10. Statistical summary of discharge and water-quality data for the low base flow seepage investigation at Oak Ridge National Laboratory

$\left[\mathrm{ft}^{3} / \mathrm{s}\right.$, cubic feet per second; $\mu \mathrm{S} / \mathrm{cm}$, microsiemens per centimeter at $25^{\circ} \mathrm{C} ;{ }^{\circ} \mathrm{C}$, degrees Celsius; --, not meaningful]

\begin{tabular}{|c|c|c|c|c|}
\hline Statistic & $\begin{array}{c}\text { Discharge } \\
\left(\mathrm{tt}^{3} / \mathrm{s}\right)\end{array}$ & pH & $\begin{array}{c}\text { Specific } \\
\text { conductance } \\
(\mu \mathrm{S} / \mathrm{cm})\end{array}$ & $\begin{array}{c}\text { Temperature } \\
\left({ }^{\circ} \mathrm{C}\right)\end{array}$ \\
\hline Number of measurements & 27 & 27 & 27 & 27 \\
\hline Maximum value & .07 & 8.1 & 516 & 23.0 \\
\hline Mean & .01 & -- & 245 & 18.2 \\
\hline Standard deviation & .02 & .4 & 103 & 2.0 \\
\hline 25th percentile & 0 & 7.2 & 197 & 17.0 \\
\hline Median & 0 & 7.4 & 259 & 18.5 \\
\hline 75th percentile & .01 & 7.5 & 275 & 19.5 \\
\hline Statistic & $\begin{array}{c}\text { Discharge } \\
\left(\mathrm{ft}^{3} / \mathrm{s}\right)\end{array}$ & pH & $\begin{array}{c}\text { Specific } \\
\text { conductance } \\
(\mu S / \mathrm{cm})\end{array}$ & $\begin{array}{c}\text { Temperature } \\
\left({ }^{\circ} \mathrm{C}\right)\end{array}$ \\
\hline Number of measurements & 39 & 39 & 40 & 40 \\
\hline Number of missed measurements & ${ }^{a} 1$ & 1 & 0 & 0 \\
\hline Minimum value & 0 & 6.6 & 87 & 13.0 \\
\hline Maximum value & .34 & 8.0 & 572 & 20.5 \\
\hline Mean & .02 & -- & 315 & 16.0 \\
\hline Standard deviation & .06 & .3 & 113 & 2.0 \\
\hline
\end{tabular}

a Two adjacent springs, 05070 and $05070 \mathrm{a}$, were quantified with one discharge measurement. 
springs, and stream-measurement sites. A total of 822 sites were identified, which consisted of 279 seeps, 68 springs, and 475 stream-measurement sites.

The high base flow seepage investigation was conducted from April 29 through May 3, 1993, and from May 7 to May 10, 1993. Measurements were made at 604 of the 822 sites identified in the reconnaissance. At 236 of the 604 sites with flowing water, flow was insufficient to measure and was reported as zero with a notation that the flow was less than the minimum reportable discharge $\left(0.005 \mathrm{ft}^{3} / \mathrm{s}\right)$. Flow rates ranged from 0 to $2.15 \mathrm{ft}^{3} / \mathrm{s}$ for the streams, from 0 to $0.07 \mathrm{ft}^{3} / \mathrm{s}$ for the seeps, and from 0 to $0.55 \mathrm{ft}^{3} / \mathrm{s}$ for the springs. Values for $\mathrm{pH}$ ranged from 5.1 to 8.5 for the streams, from 4.8 to 8.3 for the seeps, and from 5.9 to 8.0 for the springs. Specific conductance ranged from 21 to $1,004 \mu \mathrm{S} / \mathrm{cm}$ for the streams, 14 to $687 \mu \mathrm{S} / \mathrm{cm}$ for the seeps, and from 28 to $589 \mu \mathrm{S} / \mathrm{cm}$ for the springs.

Two stream reaches, one on Center Seven Tributary to Melton Branch and one on Bearden Creek, were intensively sampled in conjunction with the high base flow seepage investigation. On April 30, 1993, at Center Seven Tributary to Melton Branch, the $\mathrm{pH}$ ranged from 6.4 to 7.6; specific conductance, from 121 to $265 \mu \mathrm{S} / \mathrm{cm}$; and temperature, from 11.5 to $20.0^{\circ} \mathrm{C}$. On May 8, 1993, at Bearden Creek, the $\mathrm{pH}$ ranged from 7.7 to 8.0; specific conductance, from 371 to $458 \mu \mathrm{S} / \mathrm{cm}$; and temperature, from 14.0 to $22.0^{\circ} \mathrm{C}$.

The low base flow investigation was conducted from August 8 through August 10,1993. The seeps and springs that were flowing during the high base flow seepage investigation were revisited. Two addi- tional seeps and two springs were identified during the low base flow investigation. At 19 seep sites and 20 spring sites, the flow rate was reported as zero with a notation that the flow was less than the minimum reportable discharge $\left(0.005 \mathrm{ft}^{3} / \mathrm{s}\right)$. Twenty-seven of the 147 seeps visited were flowing and had discharge ranging from 0 to $0.07 \mathrm{ft}^{3} / \mathrm{s} ; \mathrm{pH}$, from 6.7 to 8.1 ; specific conductance, from 59 to $516 \mu \mathrm{S} / \mathrm{cm}$; and temperature, from 14.0 to $23.0^{\circ} \mathrm{C}$. Forty of the 61 springs revisited were flowing, and had discharge ranging from 0 to $0.34 \mathrm{ft}^{3} / \mathrm{s} ; \mathrm{pH}$, from 6.6 to 8.0 ; specific conductance, from 87 to $572 \mu \mathrm{S} / \mathrm{cm}$; and temperature, from 13.0 to $20.5^{\circ} \mathrm{C}$.

\section{SELECTED REFERENCES}

Hatcher, R.D., Lemiszki, P.J., Dreier, R.B., Ketelle, R.H., Lee, R.R., Leitzke, D.A., McMaster, W.M., Foreman, J.L., and Lee, S.Y., 1992, Status report on the geology of the Oak Ridge Reservation: U.S. Department of Energy, Oak Ridge National Laboratory (Report) ORNL/TM-12074, $247 \mathrm{p}$.

McMaster, W.M., 1967, Hydrologic data for the Oak Ridge area Tennessee: U.S. Geological Survey Water-Supply Paper 1839-N, $90 \mathrm{p}$.

Mulholland, P.J., 1991, Effects of flowpath variation on the hydrogeochemical response of Walker Branch watershed to storms, in Sale, M.J., and Presley, P.M., compilers, Extended abstracts from the Fourth Tennessee Water Resources Symposium, Knoxville, Tenn., 1991: American Water Resources Association p. 63-67. 
Table 2. Coordinates for seeps, springs, and stream-measurement sites at Oak Ridge National Laboratory

[Method: GPS, global positioning system; DIG, digitized; a, second site identified at the same location; Latitude and Longitude in degrees, minutes, and seconds]

\begin{tabular}{|c|c|c|c|c|c|c|c|c|c|}
\hline \multirow{3}{*}{$\begin{array}{c}\begin{array}{c}\text { Site } \\
\text { number }\end{array} \\
01025\end{array}$} & \multicolumn{2}{|c|}{ ORNL coordinates } & \multicolumn{6}{|c|}{ State plane coordinates } & \multirow{3}{*}{$\begin{array}{c}\text { Method } \\
\text { GPS }\end{array}$} \\
\hline & \multirow{2}{*}{$\begin{array}{l}\text { East } \\
15823\end{array}$} & \multirow{2}{*}{$\begin{array}{l}\text { North } \\
21678\end{array}$} & \multicolumn{3}{|c|}{ Latitude } & \multicolumn{3}{|c|}{ Longitude } & \\
\hline & & & 35 & 54 & 9.2 & -84 & 21 & 32.2 & \\
\hline 01030 & 15841 & 21681 & 35 & 54 & 9.3 & -84 & 21 & 32.0 & GPS \\
\hline 01032 & 15951 & 21868 & 35 & 54 & 11.5 & -84 & 21 & 32.2 & DIG \\
\hline 01034 & 15919 & 22185 & 35 & 54 & 13.9 & -84 & 21 & 34.7 & DIG \\
\hline 01035 & 16163 & 22037 & 35 & 54 & 14.0 & -84 & 21 & 31.2 & GPS \\
\hline 01040 & 16231 & 22029 & 35 & 54 & 14.3 & -84 & 21 & 30.5 & GPS \\
\hline 01045 & 15941 & 22506 & 35 & 54 & 16.7 & -84 & 21 & 36.6 & GPS \\
\hline 01050 & 15811 & 22622 & 35 & 54 & 16.9 & -84 & 21 & 38.7 & GPS \\
\hline 01055 & 16497 & 22064 & 35 & 54 & 16.1 & -84 & 21 & 28.0 & DIG \\
\hline 01320 & 16818 & 22645 & 35 & 54 & 22.6 & -84 & 21 & 28.7 & GPS \\
\hline 01325 & 16826 & 22901 & 35 & 54 & 24.8 & -84 & 21 & 30.3 & GPS \\
\hline 01330 & 16711 & 22878 & 35 & 54 & 24.0 & -84 & 21 & 31.3 & GPS \\
\hline 01335 & 16705 & 23044 & 35 & 54 & 25.3 & -84 & 21 & 32.5 & GPS \\
\hline 01340 & 17002 & 23452 & 35 & 54 & 30.3 & -84 & 21 & 32.3 & GPS \\
\hline 01395 & 16846 & 22472 & 35 & 54 & 21.4 & -84 & 21 & 27.2 & GPS \\
\hline 01397 & 16874 & 22429 & 35 & 54 & 21.2 & -84 & 21 & 26.7 & DIG \\
\hline 01400 & 18148 & 22513 & 35 & 54 & 28.9 & -84 & 21 & 14.4 & GPS \\
\hline 02160 & 17304 & 20481 & 35 & 54 & 7.5 & -84 & 21 & 9.1 & GPS \\
\hline 02165 & 17636 & 20616 & 35 & 54 & 10.5 & -84 & 21 & 6.7 & GPS \\
\hline 02170 & 17847 & 20693 & 35 & 54 & 12.3 & -84 & 21 & 5.1 & GPS \\
\hline 02175 & 18265 & 20755 & 35 & 54 & 15.1 & -84 & 21 & 1.3 & GPS \\
\hline 02180 & 18544 & 20768 & 35 & 54 & 16.7 & -84 & 20 & 58.6 & GPS \\
\hline 02185 & 18694 & 20793 & 35 & 54 & 17.7 & -84 & 20 & 57.2 & GPS \\
\hline 02186 & 18314 & 20745 & 35 & 54 & 15.3 & -84 & 21 & 0.7 & DIG \\
\hline 02190 & 18803 & 20908 & 35 & 54 & 19.3 & -84 & 20 & 56.9 & GPS \\
\hline 02194 & 19175 & 20205 & 35 & 54 & 15.6 & -84 & 20 & 48.4 & DIG \\
\hline 02195 & 19205 & 20959 & 35 & 54 & 21.9 & -84 & 20 & 53.2 & GPS \\
\hline 02200 & 19745 & 21099 & 35 & 54 & 26.0 & -84 & 20 & 48.7 & DIG \\
\hline 02205 & 19873 & 21310 & 35 & 54 & 28.5 & -84 & 20 & 48.8 & DIG \\
\hline 02211 & 19783 & 21371 & 35 & 54 & 28.5 & -84 & 20 & 50.1 & DIG \\
\hline 02212 & 20024 & 21817 & 35 & 54 & 33.5 & -84 & 20 & 50.7 & DIG \\
\hline 02214 & 20065 & 21947 & 35 & 54 & 34.8 & -84 & 20 & 51.2 & DIG \\
\hline 02215 & 19742 & 21393 & 35 & 54 & 28.4 & -84 & 20 & 50.7 & DIG \\
\hline 02220 & 19767 & 21732 & 35 & 54 & 31.4 & -84 & 20 & 52.7 & DIG \\
\hline 02225 & 19905 & 21964 & 35 & 54 & 34.0 & -84 & 20 & 52.9 & DIG \\
\hline 02230 & 19966 & 22014 & 35 & 54 & 34.8 & -84 & 20 & 52.6 & DIG \\
\hline 02235 & 19859 & 22271 & 35 & 54 & 36.3 & -84 & 20 & 55.5 & DIG \\
\hline 02240 & 19556 & 22704 & 35 & 54 & 38.2 & -84 & 21 & 1.4 & DIG \\
\hline 02245 & 19930 & 22418 & 35 & 54 & 37.9 & -84 & 20 & 55.7 & DIG \\
\hline 02250 & 19946 & 22363 & 35 & 54 & 37.5 & -84 & 20 & 55.2 & DIG \\
\hline 02255 & 20344 & 22646 & 35 & 54 & 42.1 & -84 & 20 & 53.1 & DIG \\
\hline 02260 & 20475 & 22759 & 35 & 54 & 43.7 & -84 & 20 & 52.5 & DIG \\
\hline 02265 & 19768 & 21075 & 35 & 54 & 26.0 & -84 & 20 & 48.3 & DIG \\
\hline 02266 & 20221 & 20377 & 35 & 54 & 22.7 & -84 & 20 & 39.0 & DIG \\
\hline 02275 & 20374 & 21139 & 35 & 54 & 29.8 & -84 & 20 & 42.6 & DIG \\
\hline 02280 & 20949 & 21180 & 35 & 54 & 33.3 & -84 & 20 & 37.1 & DIG \\
\hline 02285 & 21324 & 21283 & 35 & 54 & 36.3 & -84 & 20 & 34.0 & DIG \\
\hline 02290 & 21586 & 21419 & 35 & 54 & 38.8 & -84 & 20 & 32.3 & GPS \\
\hline 02295 & 21805 & 21779 & 35 & 54 & 43.0 & -84 & 20 & 32.5 & GPS \\
\hline 02300 & 21767 & 22013 & 35 & 54 & 44.7 & -84 & 20 & 34.4 & GPS \\
\hline
\end{tabular}


Table 2. Coordinates for seeps, springs, and stream-measurement sites at Oak Ridge National Laboratory--Continued

\begin{tabular}{|c|c|c|c|c|c|c|c|c|c|}
\hline \multirow{3}{*}{$\begin{array}{c}\begin{array}{c}\text { Site } \\
\text { number }\end{array} \\
02305\end{array}$} & \multicolumn{2}{|c|}{ ORNL coordinates } & \multicolumn{6}{|c|}{ State plane coordinates } & \multirow{3}{*}{$\begin{array}{c}\text { Mothod } \\
\text { GPS }\end{array}$} \\
\hline & \multirow{2}{*}{$\begin{array}{l}\text { East } \\
21837\end{array}$} & \multirow{2}{*}{$\frac{\text { North }}{22136}$} & \multicolumn{3}{|c|}{ Latítude } & \multicolumn{3}{|c|}{ Longitude } & \\
\hline & & & 35 & 54 & 46.1 & -84 & 20 & 34.6 & \\
\hline 02310 & 21924 & 22122 & 35 & 54 & 46.4 & -84 & 20 & 33.6 & GPS \\
\hline 02315 & 21714 & 22041 & 35 & 54 & 44.6 & -84 & 20 & 35.2 & DIG \\
\hline 02320 & 21574 & 22288 & 35 & 54 & 45.9 & -84 & 20 & 38.2 & DIG \\
\hline 02325 & 21275 & 22782 & 35 & 54 & 48.3 & -84 & 20 & 44.6 & DIG \\
\hline 02330 & 21408 & 23313 & 35 & 54 & 53.4 & -84 & 20 & 46.9 & DIG \\
\hline 02335 & 21584 & 22574 & 35 & 54 & 48.3 & -84 & 20 & 40.1 & DIG \\
\hline 02341 & 21780 & 21374 & 35 & 54 & 39.5 & -84 & 20 & 30.0 & DIG \\
\hline 02350 & 22002 & 21299 & 35 & 54 & 40.1 & -84 & 20 & 27.2 & GPS \\
\hline 02355 & 21966 & 20733 & 35 & 54 & 35.3 & -84 & 20 & 23.8 & DIG \\
\hline 02356 & 22140 & 20317 & 35 & 54 & 32.8 & -84 & 20 & 19.2 & DIG \\
\hline 02515 & 22337 & 21441 & 35 & 54 & 43.1 & -84 & 20 & 24.8 & GPS \\
\hline 02520 & 22670 & 21535 & 35 & 54 & 45.7 & -84 & 20 & 22.1 & GPS \\
\hline 02525 & 23601 & 20599 & 35 & 54 & 43.2 & -84 & 20 & 6.4 & GPS \\
\hline 02527 & 23619 & 21007 & 35 & 54 & 46.6 & -84 & 20 & 8.9 & GPS \\
\hline 02528 & 23434 & 21042 & 35 & 54 & 45.9 & -84 & 20 & 11.0 & DIG \\
\hline 02530 & 23360 & 20805 & 35 & 54 & 43.5 & -84 & 20 & 10.2 & GPS \\
\hline 02535 & 23405 & 21290 & 35 & 54 & 47.8 & -84 & 20 & 13.0 & DIG \\
\hline 02540 & 23309 & 21320 & 35 & 54 & 47.5 & -84 & 20 & 14.2 & GPS \\
\hline 02545 & 23070 & 21623 & 35 & 54 & 48.6 & -84 & 20 & 18.6 & GPS \\
\hline 02550 & 23089 & 21658 & 35 & 54 & 49.0 & -84 & 20 & 18.7 & GPS \\
\hline 02555 & 23381 & 22173 & 35 & 54 & 54.9 & -84 & 20 & 19.2 & GPS \\
\hline 02556 & 23330 & 22627 & 35 & 54 & 58.3 & -84 & 20 & 22.8 & GPS \\
\hline 02557 & 23307 & 22787 & 35 & 54 & 59.5 & -84 & 20 & 24.1 & GPS \\
\hline 02559 & 23248 & 23216 & 35 & 55 & 2.7 & -84 & 20 & 27.6 & GPS \\
\hline 02560 & 23308 & 21731 & 35 & 54 & 50.8 & -84 & 20 & 17.0 & DIG \\
\hline 02565 & 23321 & 21711 & 35 & 54 & 50.8 & -84 & 20 & 16.7 & GPS \\
\hline 02570 & 24361 & 20689 & 35 & 54 & 48.1 & -84 & 19 & 59.3 & GPS \\
\hline 02575 & 24300 & 20859 & 35 & 54 & 49.1 & -84 & 20 & 1.0 & GPS \\
\hline 02580 & 24092 & 21154 & 35 & 54 & 50.4 & -84 & 20 & 5.1 & GPS \\
\hline 02585 & 23863 & 21394 & 35 & 54 & 51.1 & -84 & 20 & 9.1 & GPS \\
\hline 02590 & 23682 & 21748 & 35 & 54 & 53.0 & -84 & 20 & 13.3 & DIG \\
\hline 02595 & 23665 & 21779 & 35 & 54 & 53.2 & -84 & 20 & 13.7 & GPS \\
\hline 02600 & 23991 & 22026 & 35 & 54 & 57.0 & -84 & 20 & 12.1 & GPS \\
\hline 02605 & 24475 & 22076 & 35 & 55 & 0.1 & -84 & 20 & 7.5 & GPS \\
\hline 02610 & 23970 & 22242 & 35 & 54 & 58.7 & -84 & 20 & 13.7 & GPS \\
\hline 02615 & 24182 & 22612 & 35 & 55 & 2.9 & -84 & 20 & 14.1 & GPS \\
\hline 02620 & 24231 & 22679 & 35 & 55 & 3.7 & -84 & 20 & 14.1 & GPS \\
\hline 02625 & 24689 & 22957 & 35 & 55 & 8.5 & -84 & 20 & 11.3 & GPS \\
\hline 02630 & 24620 & 23398 & 35 & 55 & 11.8 & -84 & 20 & 15.0 & DIG \\
\hline 02635 & 24758 & 22903 & 35 & 55 & 8.5 & -84 & 20 & 10.2 & GPS \\
\hline 02640 & 24874 & 23181 & 35 & 55 & 11.4 & -84 & 20 & 11.0 & DIG \\
\hline 02645 & 25024 & 23093 & 35 & 55 & 11.5 & -84 & 20 & 8.8 & DIG \\
\hline 02680 & 25070 & 20737 & 35 & 54 & 52.4 & -84 & 19 & 52.5 & DIG \\
\hline 03001 & 29735 & 20624 & 35 & 55 & 17.1 & -84 & 19 & 4.6 & GPS \\
\hline 03003 & 29642 & 20810 & 35 & 55 & 18.1 & -84 & 19 & 6.8 & GPS \\
\hline 03005 & 29665 & 21338 & 35 & 55 & 22.6 & -84 & 19 & 10.1 & GPS \\
\hline 03050 & 29673 & 22571 & 35 & 55 & 32.8 & -84 & 19 & 18.3 & GPS \\
\hline 03060 & 29489 & 23155 & 35 & 55 & 36.6 & -84 & 19 & 24.2 & .GPS \\
\hline 03065 & 29572 & 23482 & 35 & 55 & 39.7 & -84 & 19 & 25.5 & GPS \\
\hline 03070 & 29566 & 23534 & 35 & 55 & 40.1 & -84 & 19 & 25.9 & GPS \\
\hline 03075 & 29582 & 23549 & 35 & 55 & 40.3 & -84 & 19 & 25.9 & GPS \\
\hline 03080 & 29621 & 23559 & 35 & 55 & 40.6 & -84 & 19 & 25.6 & GPS \\
\hline
\end{tabular}


Table 2. Coordinates for seeps, springs, and stream-measurement sites at Oak Ridge National Laboratory--Continued

\begin{tabular}{|c|c|c|c|c|c|c|c|c|c|}
\hline \multirow{3}{*}{$\begin{array}{c}\begin{array}{c}\text { Site } \\
\text { number }\end{array} \\
03085\end{array}$} & \multicolumn{2}{|c|}{ ORNL coordinates } & \multicolumn{6}{|c|}{ State plane coordinates } & \multirow{3}{*}{$\begin{array}{c}\text { Method } \\
\text { GPS }\end{array}$} \\
\hline & \multirow{2}{*}{$\begin{array}{l}\text { East } \\
29616\end{array}$} & \multirow{2}{*}{$\begin{array}{l}\text { North } \\
23656\end{array}$} & \multicolumn{3}{|c|}{ Latitude } & \multicolumn{3}{|c|}{ Longitude } & \\
\hline & & & 35 & 55 & 41.4 & -84 & 19 & 26.3 & \\
\hline 03090 & 29646 & 23745 & 35 & 55 & 42.3 & -84 & 19 & 26.6 & GPS \\
\hline 03095 & 29686 & 23772 & 35 & 55 & 42.7 & -84 & 19 & 26.3 & GPS \\
\hline 03100 & 29696 & 23853 & 35 & 55 & 43.4 & -84 & 19 & 26.8 & GPS \\
\hline 03110 & 29568 & 23696 & 35 & 55 & 41.4 & -84 & 19 & 27.0 & GPS \\
\hline 03115 & 29585 & 23815 & 35 & 55 & 42.5 & -84 & 19 & 27.7 & GPS \\
\hline 03485 & 29559 & 24259 & 35 & 55 & 46.0 & -84 & 19 & 30.9 & GPS \\
\hline 03490 & 29528 & 24436 & 35 & 55 & 47.3 & -84 & 19 & 32.4 & GPS \\
\hline 03495 & 29498 & 24463 & 35 & 55 & 47.3 & -84 & 19 & 32.9 & GPS \\
\hline 03500 & 29583 & 24778 & 35 & 55 & 50.4 & -84 & 19 & 34.2 & GPS \\
\hline 03510 & 29259 & 20995 & 35 & 55 & 17.5 & -84 & 19 & 11.9 & DIG \\
\hline 03515 & 29118 & 21099 & 35 & 55 & 17.6 & -84 & 19 & 14.0 & GPS \\
\hline 03520 & 28941 & 21262 & 35 & 55 & 18.0 & -84 & 19 & 16.9 & GPS \\
\hline 03525 & 28838 & 21390 & 35 & 55 & 18.5 & -84 & 19 & 18.8 & GPS \\
\hline 03530 & 28878 & 21596 & 35 & 55 & 20.4 & -84 & 19 & 19.8 & GPS \\
\hline 03535 & 28611 & 21558 & 35 & 55 & 18.6 & -84 & 19 & 22.2 & GPS \\
\hline 03537 & 28291 & 21138 & 35 & 55 & 13.4 & -84 & 19 & 22.6 & GPS \\
\hline 03538 & 28257 & 21038 & 35 & 55 & 12.4 & -84 & 19 & 22.3 & GPS \\
\hline 03540 & 28446 & 21788 & 35 & 55 & 19.6 & -84 & 19 & 25.4 & GPS \\
\hline 03545 & 28342 & 21945 & 35 & 55 & 20.3 & -84 & 19 & 27.6 & GPS \\
\hline 03550 & 28156 & 22033 & 35 & 55 & 20.0 & -84 & 19 & 30.0 & GPS \\
\hline 03555 & 28103 & 22106 & 35 & 55 & 20.3 & -84 & 19 & 31.1 & GPS \\
\hline 03560 & 28062 & 22164 & 35 & 55 & 20.6 & -84 & 19 & 31.9 & GPS \\
\hline 03565 & 28099 & 22551 & 35 & 55 & 23.9 & -84 & 19 & 34.1 & GPS \\
\hline 03570 & 28176 & 22646 & 35 & 55 & 25.1 & -84 & 19 & 34.0 & GPS \\
\hline 03575 & 28235 & 22739 & 35 & 55 & 26.2 & -84 & 19 & 34.0 & GPS \\
\hline 03580 & 28281 & 23094 & 35 & 55 & 29.4 & -84 & 19 & 35.9 & GPS \\
\hline 03585 & 28382 & 21775 & 35 & 55 & 19.1 & -84 & 19 & 26.0 & GPS \\
\hline 03590 & 28206 & 21815 & 35 & 55 & 18.5 & -84 & 19 & 28.1 & GPS \\
\hline 03591 & 27794 & 21521 & 35 & 55 & 13.8 & -84 & 19 & 30.2 & GPS \\
\hline 03592 & 27657 & 21483 & 35 & 55 & 12.7 & -84 & 19 & 31.4 & GPS \\
\hline 03593 & 26969 & 21396 & 35 & 55 & 8.2 & -84 & 19 & 37.7 & GPS \\
\hline 03594 & 27641 & 21382 & 35 & 55 & 11.8 & -84 & 19 & 30.8 & GPS \\
\hline 03595 & 27648 & 21372 & 35 & 55 & 11.8 & -84 & 19 & 30.7 & GPS \\
\hline 03598 & 27671 & 21367 & 35 & 55 & 11.9 & -84 & 19 & 30.4 & GPS \\
\hline 03600 & 27925 & 21070 & 35 & 55 & 10.8 & -84 & 19 & 25.9 & GPS \\
\hline 03607 & 27629 & 21220 & 35 & 55 & 10.4 & -84 & 19 & 29.9 & GPS \\
\hline 03610 & 27615 & 21093 & 35 & 55 & 9.3 & -84 & 19 & 29.1 & GPS \\
\hline 03615 & 27766 & 20795 & 35 & 55 & 7.7 & -84 & 19 & 25.6 & GPS \\
\hline 03620 & 27760 & 20643 & 35 & 55 & 6.4 & -84 & 19 & 24.6 & GPS \\
\hline 03625 & 27792 & 21965 & 35 & 55 & 17.4 & -84 & 19 & 33.3 & GPS \\
\hline 03630 & 27718 & 22235 & 35 & 55 & 19.2 & -84 & 19 & 35.8 & GPS \\
\hline 03635 & 27652 & 22639 & 35 & 55 & 22.2 & -84 & 19 & 39.2 & GPS \\
\hline 03640 & 27617 & 22883 & 35 & 55 & 24.0 & -84 & 19 & 41.2 & GPS \\
\hline 03645 & 27469 & 23241 & 35 & 55 & 26.1 & -84 & 19 & 45.2 & GPS \\
\hline 03648 & 27141 & 23053 & 35 & 55 & 22.8 & -84 & 19 & 47.2 & GPS \\
\hline 03650 & 27146 & 23294 & 35 & 55 & 24.8 & -84 & 19 & 48.8 & GPS \\
\hline 03654 & 26811 & 23263 & 35 & 55 & 22.7 & -84 & 19 & 51.9 & GPS \\
\hline 03655 & 26675 & 23715 & 35 & 55 & 25.7 & -84 & 19 & 56.4 & GPS \\
\hline 03660 & 27828 & 23283 & 35 & 55 & 28.5 & -84 & 19 & 41.8 & GPS \\
\hline 03670 & 27898 & 23903 & 35 & 55 & 34.0 & -84 & 19 & 45.3 & GPS \\
\hline 03672 & 27888 & 23968 & 35 & 55 & 34.4 & -84 & 19 & 45.8 & GPS \\
\hline 03673 & 27856 & 24161 & 35 & 55 & 35.8 & -84 & 19 & 47.5 & DIG \\
\hline
\end{tabular}


Table 2. Coordinates for seeps, springs, and stream-measurement sites at Oak Ridge National Laboratory--Continued

\begin{tabular}{|c|c|c|c|c|c|c|c|c|c|}
\hline \multirow{3}{*}{$\begin{array}{c}\begin{array}{c}\text { Site } \\
\text { number }\end{array} \\
03674\end{array}$} & \multicolumn{2}{|c|}{ ORNL coordinates } & \multicolumn{6}{|c|}{ State piane coordinates } & \multirow{3}{*}{$\begin{array}{c}\text { Method } \\
\text { GPS }\end{array}$} \\
\hline & \multirow{2}{*}{$\begin{array}{l}\text { East } \\
27885\end{array}$} & \multirow{2}{*}{$\begin{array}{l}\text { North } \\
24139\end{array}$} & \multicolumn{3}{|c|}{ Latitude } & \multicolumn{3}{|c|}{ Longltude } & \\
\hline & & & 35 & 55 & 35.8 & -84 & 19 & 47.0 & \\
\hline 03675 & 27820 & 24212 & 35 & 55 & 36.0 & -84 & 19 & 48.2 & GPS \\
\hline 03680 & 27330 & 22136 & 35 & 55 & 16.3 & -84 & 19 & 39.1 & GPS \\
\hline 03685 & 26839 & 22143 & 35 & 55 & 13.7 & -84 & 19 & 44.1 & GPS \\
\hline 03686 & 26791 & 22137 & 35 & 55 & 13.3 & -84 & 19 & 44.5 & DIG \\
\hline 03690 & 25827 & 23039 & 35 & 55 & 15.5 & -84 & 20 & 0.4 & GPS \\
\hline 03695 & 25609 & 23422 & 35 & 55 & 17.4 & -84 & 20 & 5.2 & GPS \\
\hline 03697 & 25732 & 23758 & 35 & 55 & 20.8 & -84 & 20 & 6.2 & GPS \\
\hline 03698 & 25745 & 23846 & 35 & 55 & 21.6 & -84 & 20 & 6.7 & GPS \\
\hline 03699 & 25873 & 23994 & 35 & 55 & 23.6 & -84 & 20 & 6.4 & DIG \\
\hline 03700 & 26719 & 22030 & 35 & 55 & 12.1 & -84 & 19 & 44.5 & GPS \\
\hline 03705 & 26623 & 21648 & 35 & 55 & 8.4 & -84 & 19 & 42.9 & GPS \\
\hline 03710 & 26234 & 21360 & 35 & 55 & 3.9 & -84 & 19 & 44.9 & GPS \\
\hline 03715 & 26248 & 20932 & 35 & 55 & 0.5 & -84 & 19 & 41.9 & GPS \\
\hline 03718 & 26368 & 20726 & 35 & 54 & 59.4 & -84 & 19 & 39.3 & DIG \\
\hline 03720 & 26368 & 20726 & 35 & 54 & 59.2 & -84 & 19 & 39.3 & GPS \\
\hline 04003 & 31665 & 23456 & 35 & 55 & 51.0 & -84 & 19 & 4.2 & GPS \\
\hline 04006 & 31927 & 23465 & 35 & 55 & 52.5 & -84 & 19 & 1.6 & GPS \\
\hline 04009 & 32120 & 23687 & 35 & 55 & 55.4 & -84 & 19 & 1.2 & GPS \\
\hline 04012 & 32151 & 23723 & 35 & 55 & 55.9 & -84 & 19 & 1.1 & GPS \\
\hline 04015 & 32123 & 23778 & 35 & 55 & 56.2 & -84 & 19 & 1.8 & GPS \\
\hline 04018 & 32112 & 23774 & 35 & 55 & 56.1 & -84 & 19 & 1.8 & GPS \\
\hline 04021 & 32116 & 23815 & 35 & 55 & 56.4 & -84 & 19 & 2.1 & GPS \\
\hline 04024 & 32144 & 23908 & 35 & 55 & 57.3 & -84 & 19 & 2.4 & GPS \\
\hline 04027 & 32151 & 24033 & 35 & 55 & 58.4 & -84 & 19 & 3.2 & DIG \\
\hline 04030 & 32086 & 24125 & 35 & 55 & 58.8 & -84 & 19 & 4.5 & GPS \\
\hline 04033 & 32304 & 24116 & 35 & 55 & 59.9 & -84 & 19 & 2.2 & GPS \\
\hline 04036 & 33054 & 24937 & 35 & 56 & 10.8 & -84 & 19 & 0.2 & GPS \\
\hline 04037 & 33078 & 24941 & 35 & 56 & 11.0 & -84 & 19 & 0.0 & DIG \\
\hline 04039 & 33118 & 24949 & 35 & 56 & 11.3 & -84 & 18 & 59.6 & DIG \\
\hline 04051 & 32404 & 24548 & 35 & 56 & 4.0 & -84 & 19 & 4.1 & GPS \\
\hline 04054 & 32440 & 24870 & 35 & 56 & 6.9 & -84 & 19 & 5.9 & GPS \\
\hline 04057 & 32166 & 24735 & 35 & 56 & 4.3 & -84 & 19 & 7.8 & GPS \\
\hline 04060 & 32083 & 24918 & 35 & 56 & 5.3 & -84 & 19 & 9.9 & GPS \\
\hline 05005 & 34698 & 21709 & 35 & 55 & 53.3 & -84 & 18 & 21.7 & DIG \\
\hline 05010 & 34867 & 21843 & 35 & 55 & 55.3 & -84 & 18 & 20.9 & DIG \\
\hline 05015 & 35033 & 21863 & 35 & 55 & 56.4 & -84 & 18 & 19.4 & DIG \\
\hline 05020 & 35037 & 22818 & 35 & 56 & 4.3 & -84 & 18 & 25.8 & DIG \\
\hline 05035 & 35271 & 23096 & 35 & 56 & 7.9 & -84 & 18 & 25.3 & DIG \\
\hline 05040 & 35678 & 23189 & 35 & 56 & 10.9 & -84 & 18 & 21.9 & DIG \\
\hline 05045 & 36171 & 23374 & 35 & 56 & 15.1 & -84 & 18 & 18.1 & DIG \\
\hline 05050 & 35021 & 23534 & 35 & 56 & 10.1 & -84 & 18 & 30.8 & DIG \\
\hline 05055 & 35296 & 21876 & 35 & 55 & 58.0 & -84 & 18 & 16.8 & DIG \\
\hline 05060 & 35354 & 21594 & 35 & 55 & 56.0 & -84 & 18 & 14.3 & GPS \\
\hline 05065 & 35331 & 21504 & 35 & 55 & 55.1 & -84 & 18 & 14.0 & GPS \\
\hline 05070 & 35426 & 21367 & 35 & 55 & 54.5 & -84 & 18 & 12.1 & GPS \\
\hline $05070 \mathrm{a}$ & 35426 & 21367 & 35 & 55 & 54.5 & -84 & 18 & 12.1 & DIG \\
\hline 05075 & 35295 & 21346 & 35 & 55 & 53.6 & -84 & 18 & 13.3 & GPS \\
\hline 05077 & 35260 & 21196 & 35 & 55 & 52.2 & -84 & 18 & 12.6 & GPS \\
\hline 05080 & 35081 & 20964 & 35 & 55 & 49.3 & -84 & 18 & 12.8 & GPS \\
\hline 05085 & 35373 & 21617 & 35 & 55 & 56.3 & -84 & 18 & 14.3 & GPS \\
\hline 05090 & 35761 & 21333 & 35 & 55 & 56.1 & -84 & 18 & 8.5 & GPS \\
\hline 05095 & 35585 & 20726 & 35 & 55 & 50.1 & -84 & 18 & 6.1 & GPS \\
\hline
\end{tabular}


Table 2. Coordinates for seeps, springs, and stream-measurement sites at Oak Ridge National Laboratory--Continued

\begin{tabular}{|c|c|c|c|c|c|c|c|c|c|}
\hline \multirow{3}{*}{$\begin{array}{c}\begin{array}{c}\text { Site } \\
\text { number }\end{array} \\
05100\end{array}$} & \multicolumn{2}{|c|}{ ORNL coordinates } & \multicolumn{6}{|c|}{ State plane coordinates. } & \multirow{3}{*}{$\begin{array}{c}\text { Method } \\
\text { GPS }\end{array}$} \\
\hline & \multirow{2}{*}{$\begin{array}{l}\text { East } \\
35571\end{array}$} & \multirow{2}{*}{$\begin{array}{l}\text { North } \\
20449\end{array}$} & \multicolumn{3}{|c|}{ Latitude } & \multicolumn{3}{|c|}{ Longitude } & \\
\hline & & & 35 & 55 & 47.8 & -84 & 18 & 4.4 & \\
\hline 05105 & 36112 & 20805 & 35 & 55 & 53.7 & -84 & 18 & 1.3 & GPS \\
\hline 05108 & 35841 & 20534 & 35 & 55 & 49.9 & -84 & 18 & 2.3 & GPS \\
\hline 05110 & 36330 & 20447 & 35 & 55 & 51.9 & -84 & 17 & 56.7 & GPS \\
\hline 05112 & 36135 & 20096 & 35 & 55 & 48.0 & -84 & 17 & 56.3 & GPS \\
\hline 05115 & 36399 & 20181 & 35 & 55 & 50.1 & -84 & 17 & 54.2 & GPS \\
\hline 05120 & 36425 & 20213 & 35 & 55 & 50.5 & -84 & 17 & 54.2 & GPS \\
\hline 05125 & 36835 & 20412 & 35 & 55 & 54.4 & -84 & 17 & 51.4 & GPS \\
\hline 05130 & 36946 & 20130 & 35 & 55 & 52.7 & -84 & 17 & 48.4 & GPS \\
\hline 05135 & 37057 & 20065 & 35 & 55 & 52.8 & -84 & 17 & 46.8 & GPS \\
\hline 05150 & 37376 & 20029 & 35 & 55 & 54.2 & -84 & 17 & 43.3 & GPS \\
\hline 05155 & 35346 & 21908 & 35 & 55 & 58.5 & -84 & 18 & 16.5 & DIG \\
\hline 05160 & 35472 & 21832 & 35 & 55 & 58.6 & -84 & 18 & 17.8 & DIG \\
\hline 05165 & 35577 & 21502 & 35 & 55 & 56.4 & -84 & 18 & 11.5 & GPS \\
\hline 05170 & 36054 & 21138 & 35 & 55 & 56.1 & -84 & 18 & 4.2 & GPS \\
\hline 05175 & 36319 & 20786 & 35 & 55 & 54.6 & -84 & 17 & 59.1 & GPS \\
\hline 05180 & 35893 & 21848 & 35 & 56 & 1.0 & -84 & 18 & 10.6 & DIG \\
\hline 05182 & 36081 & 21854 & 35 & 56 & 2.1 & -84 & 18 & 8.7 & DIG \\
\hline 05183 & 36183 & 21607 & 35 & 56 & 0.6 & -84 & 18 & 6.1 & DIG \\
\hline 05184 & 36151 & 21575 & 35 & 56 & 0.2 & -84 & 18 & 6.2 & DIG \\
\hline 05188 & 36495 & 20868 & 35 & 55 & 56.3 & -84 & 17 & 57.9 & GPS \\
\hline 05190 & 36130 & 21898 & 35 & 56 & 2.7 & -84 & 18 & 8.6 & DIG \\
\hline 05192 & 36283 & 21873 & 35 & 56 & 3.4 & -84 & 18 & 6.8 & DIG \\
\hline 05195 & 36095 & 21954 & 35 & 56 & 3.0 & -84 & 18 & 9.3 & DIG \\
\hline 05197 & 36333 & 22205 & 35 & 56 & 6.4 & -84 & 18 & 8.6 & GPS \\
\hline 05199 & 36870 & 22286 & 35 & 56 & 10.0 & -84 & 18 & 3.7 & GPS \\
\hline 05200 & 36341 & 22216 & 35 & 56 & 6.5 & -84 & 18 & 8.6 & GPS \\
\hline 05209 & 36584 & 22855 & 35 & 56 & 13.1 & -84 & 18 & 10.4 & GPS \\
\hline 05212 & 36522 & 22915 & 35 & 56 & 13.3 & -84 & 18 & 11.5 & GPS \\
\hline 05218 & 36502 & 22997 & 35 & 56 & 13.8 & -84 & 18 & 12.2 & GPS \\
\hline 05219 & 36763 & 22510 & 35 & 56 & 11.3 & -84 & 18 & 6.3 & GPS \\
\hline 05221 & 36857 & 22996 & 35 & 56 & 15.8 & -84 & 18 & 8.6 & GPS \\
\hline 05224 & 36788 & 23057 & 35 & 56 & 15.9 & -84 & 18 & 9.7 & DIG \\
\hline 05227 & 36645 & 23144 & 35 & 56 & $15.8^{\circ}$ & -84 & 18 & 11.8 & GPS \\
\hline 05230 & 36704 & 23150 & 35 & 56 & 16.2 & -84 & 18 & 11.2 & GPS \\
\hline 05236 & 36844 & 23130 & 35 & 56 & 16.8 & -84 & 18 & 9.7 & DIG \\
\hline 05239 & 36806 & 23222 & 35 & 56 & 17.3 & -84 & 18 & 10.7 & GPS \\
\hline 05242 & 36863 & 23237 & 35 & 56 & 17.8 & -84 & 18 & 10.2 & DIG \\
\hline 05245 & 37257 & 22911 & 35 & 56 & 17.3 & -84 & 18 & 4.0 & DIG \\
\hline 05247 & 37593 & 23157 & 35 & 56 & 21.1 & -84 & 18 & 2.3 & GPS \\
\hline 05248 & 37483 & 23178 & 35 & 56 & 20.7 & -84 & 18 & 3.5 & GPS \\
\hline 05251 & 37516 & 23184 & 35 & 56 & 20.9 & -84 & 18 & 3.2 & GPS \\
\hline 05255 & 38494 & 23186 & 35 & 56 & 26.3 & -84 & 17 & 53.4 & DIG \\
\hline 05260 & 38908 & 23144 & 35 & 56 & 28.3 & -84 & 17 & 48.9 & DIG \\
\hline 05265 & 37872 & 23905 & 35 & 56 & 28.8 & -84 & 18 & 4.5 & GPS \\
\hline 05270 & 37886 & 23958 & 35 & 56 & 29.3 & -84 & 18 & 4.7 & GPS \\
\hline 05275 & 37743 & 24076 & 35 & 56 & 29.5 & -84 & 18 & 7.0 & DIG \\
\hline 05278 & 37477 & 24257 & 35 & 56 & 29.5 & -84 & 18 & 10.9 & DIG \\
\hline 05280 & 37442 & 24347 & 35 & 56 & 30.1 & -84 & 18 & 11.8 & DIG \\
\hline 05285 & 37234 & 24565 & 35 & 56 & 30.7 & -84 & 18 & 15.4 & DIG \\
\hline 05290 & 37899 & 24269 & 35 & 56 & 32.0 & -84 & 18 & 6.7 & GPS \\
\hline 05295 & 37907 & 24164 & 35 & 56 & 31.1 & -84 & 18 & 5.9 & GPS \\
\hline 05300 & 38037 & 24162 & 35 & 56 & 31.8 & -84 & 18 & 4.6 & GPS \\
\hline
\end{tabular}


Table 2. Coordinates for seeps, springs, and stream-measurement sites at Oak Ridge National Laboratory--Continued

\begin{tabular}{|c|c|c|c|c|c|c|c|c|c|}
\hline \multirow{3}{*}{$\begin{array}{c}\begin{array}{c}\text { Stte } \\
\text { number }\end{array} \\
05305\end{array}$} & \multicolumn{2}{|c|}{ ORNL coordinates } & \multicolumn{6}{|c|}{ State plane coordlnates } & \multirow{3}{*}{$\begin{array}{c}\text { Method } \\
\text { GPS }\end{array}$} \\
\hline & \multirow{2}{*}{$\begin{array}{l}\text { East } \\
38061\end{array}$} & \multirow{2}{*}{$\frac{\text { North }}{24270}$} & \multicolumn{3}{|c|}{ Lathude } & \multicolumn{3}{|c|}{ Longltude } & \\
\hline & & & 35 & 56 & 32.9 & -84 & 18 & 5.1 & \\
\hline 05310 & 38013 & 24394 & 35 & 56 & 33.6 & -84 & 18 & 6.4 & GPS \\
\hline 05315 & 38133 & 24317 & 35 & 56 & 33.6 & -84 & 18 & 4.7 & GPS \\
\hline 05320 & 38136 & 24382 & 35 & 56 & 34.2 & -84 & 18 & 5.1 & GPS \\
\hline 05325 & 38176 & 24390 & 35 & 56 & 34.5 & -84 & 18 & 4.7 & GPS \\
\hline 05328 & 38191 & 24361 & 35 & 56 & 34.3 & -84 & 18 & 4.4 & GPS \\
\hline 05330 & 38225 & 24438 & 35 & 56 & 35.1 & -84 & 18 & 4.6 & GPS \\
\hline 05335 & 38259 & 24453 & 35 & 56 & 35.4 & -84 & 18 & 4.3 & GPS \\
\hline 05340 & 38057 & 24137 & 35 & 56 & 31.7 & -84 & 18 & 4.2 & GPS \\
\hline 05345 & 38120 & 24182 & 35 & 56 & 32.5 & -84 & 18 & 3.9 & GPS \\
\hline 05350 & 38144 & 24168 & 35 & 56 & 32.5 & -84 & 18 & 3.6 & GPS \\
\hline 05355 & 38221 & 24144 & 35 & 56 & 32.7 & -84 & 18 & 2.6 & GPS \\
\hline 06005 & 40534 & 21137 & 35 & 56 & 20.7 & -84 & 17 & 18.9 & GPS \\
\hline 06010 & 40730 & 22300 & 35 & 56 & 31.3 & -84 & 17 & 24.8 & DIG \\
\hline 06015 & 40235 & 21600 & 35 & 56 & 22.9 & -84 & 17 & 25.0 & GPS \\
\hline 06020 & 40133 & 21716 & 35 & 56 & 23.3 & -84 & 17 & 26.9 & GPS \\
\hline 06025 & 39198 & 21350 & 35 & 56 & 15.1 & -84 & 17 & 33.8 & GPS \\
\hline 06030 & 38941 & 21200 & 35 & 56 & 12.5 & -84 & 17 & 35.4 & GPS \\
\hline 06040 & 38551 & 20861 & 35 & 56 & 7.5 & -84 & 17 & 37.1 & GPS \\
\hline 06042 & 38429 & 20712 & 35 & 56 & 5.6 & -84 & 17 & 37.3 & GPS \\
\hline 06044 & 38159 & 20449 & 35 & 56 & 2.0 & -84 & 17 & 38.2 & DIG \\
\hline 06045 & 38460 & 20613 & 35 & 56 & 5.0 & -84 & 17 & 36.3 & GPS \\
\hline 06048 & 38446 & 20474 & 35 & 56 & 3.8 & -84 & 17 & 35.5 & GPS \\
\hline 06050 & 38556 & 20168 & 35 & 56 & 1.9 & -84 & 17 & 32.3 & GPS \\
\hline 06055 & 40099 & 21754 & 35 & 56 & 23.4 & -84 & 17 & 27.5 & GPS \\
\hline 06056 & 39879 & 21815 & 35 & 56 & 22.7 & -84 & 17 & 30.1 & DIG \\
\hline 06060 & 39760 & 21992 & 35 & 56 & 23.5 & -84 & 17 & 32.5 & GPS \\
\hline 06065 & 39622 & 22063 & 35 & 56 & 23.3 & -84 & 17 & 34.4 & GPS \\
\hline 06070 & 39525 & 22188 & 35 & 56 & 23.8 & -84 & 17 & 36.2 & GPS \\
\hline 06075 & 39323 & 22420 & 35 & 56 & 24.6 & -84 & 17 & 39.8 & GPS \\
\hline 06080 & 40115 & 21802 & 35 & 56 & 23.9 & -84 & 17 & 27.6 & GPS \\
\hline 06085 & 40098 & 22257 & 35 & 56 & 27.5 & -84 & 17 & 30.9 & GPS \\
\hline 06087 & 39950 & 22978 & 35 & 56 & 32.6 & -84 & 17 & 37.3 & GPS \\
\hline 06090 & 40176 & 22759 & 35 & 56 & 32.1 & -84 & 17 & 33.5 & GPS \\
\hline 06095 & 40330 & 23035 & 35 & 56 & 35.2 & -84 & 17 & 33.8 & GPS \\
\hline 06100 & 40419 & 23242 & 35 & 56 & 37.4 & -84 & 17 & 34.3 & GPS \\
\hline 07005 & 40486 & 18938 & 35 & 56 & 2.4 & -84 & 17 & 4.5 & GPS \\
\hline 07010 & 40652 & 18757 & 35 & 56 & 1.8 & -84 & 17 & 1.6 & GPS \\
\hline 07015 & 40268 & 18858 & 35 & 56 & 0.5 & -84 & 17 & 6.2 & GPS \\
\hline 07020 & 40252 & 18231 & 35 & 55 & 55.3 & -84 & 17 & 2.1 & GPS \\
\hline 07021 & 40215 & 18284 & 35 & 55 & 55.5 & -84 & 17 & 2.8 & DIG \\
\hline 07022 & 40278 & 18341 & 35 & 55 & 56.3 & -84 & 17 & 2.6 & DIG \\
\hline 07025 & 39959 & 18752 & 35 & 55 & 57.9 & -84 & 17 & 8.6 & GPS \\
\hline 07030 & 39953 & 19111 & 35 & 56 & 0.9 & -84 & 17 & 11.1 & GPS \\
\hline 07035 & 40122 & 18187 & 35 & 55 & 54.2 & -84 & 17 & 3.1 & GPS \\
\hline 07038 & 39797 & 18206 & 35 & 55 & 52.6 & -84 & 17 & 6.5 & GPS \\
\hline 07040 & 39846 & 18227 & 35 & 55 & 53.0 & -84 & 17 & 6.2 & GPS \\
\hline 07045 & 39625 & 18656 & 35 & 55 & 55.3 & -84 & 17 & 11.3 & GPS \\
\hline 07050 & 39603 & 19052 & 35 & 55 & 58.4 & -84 & 17 & 14.2 & GPS \\
\hline 07100 & 39538 & 17501 & 35 & 55 & 45.3 & -84 & 17 & 4.4 & GPS \\
\hline 07105 & 39474 & 17465 & 35 & 55 & 44.7 & -84 & 17 & 4.8 & GPS \\
\hline 07110 & 39371 & 17152 & 35 & 55 & 41.6 & -84 & 17 & 3.7 & DIG \\
\hline 07115 & 39215 & 17628 & 35 & 55 & 44.6 & -84 & 17 & 8.5 & GPS \\
\hline
\end{tabular}


Table 2. Coordinates for seeps, springs, and stream-measurement sites at Oak Ridge National Laboratory--Continued

\begin{tabular}{|c|c|c|c|c|c|c|c|c|c|}
\hline \multirow{3}{*}{$\begin{array}{c}\begin{array}{c}\text { Site } \\
\text { number }\end{array} \\
07120\end{array}$} & \multicolumn{2}{|c|}{ ORNL coordinates } & \multicolumn{6}{|c|}{ State plane coordinates } & \multirow{3}{*}{$\frac{\text { Method }}{\text { DIG }}$} \\
\hline & \multirow{2}{*}{$\begin{array}{l}\text { East } \\
39020\end{array}$} & \multirow{2}{*}{$\frac{\text { North }}{17337}$} & \multicolumn{3}{|c|}{ Latitude } & \multicolumn{3}{|c|}{ Longitude } & \\
\hline & & & 35 & 55 & 41.1 & -84 & 17 & 8.5 & \\
\hline 07125 & 39241 & 17642 & 35 & 55 & 44.9 & -84 & 17 & 8.3 & GPS \\
\hline 07130 & 39164 & 17739 & 35 & 55 & 45.2 & -84 & 17 & 9.8 & GPS \\
\hline 07135 & 39217 & 18164 & 35 & 55 & 49.0 & -84 & 17 & 12.1 & GPS \\
\hline 07140 & 39254 & 18510 & 35 & 55 & 52.1 & -84 & 17 & 14.1 & GPS \\
\hline 07145 & 39202 & 18931 & 35 & 55 & 55.3 & -84 & 17 & 17.5 & GPS \\
\hline 07200 & 39095 & 17751 & 35 & 55 & 45.0 & -84 & 17 & 10.6 & GPS \\
\hline 07205 & 39093 & 18037 & 35 & 55 & 47.3 & -84 & 17 & 12.5 & GPS \\
\hline 07210 & 38753 & 17812 & 35 & 55 & 43.6 & -84 & 17 & 14.4 & GPS \\
\hline 07215 & 38627 & 17810 & 35 & 55 & 42.9 & -84 & 17 & 15.7 & GPS \\
\hline 07220 & 38527 & 17465 & 35 & 55 & 39.5 & -84 & 17 & 14.4 & GPS \\
\hline 07225 & 38350 & 17370 & 35 & 55 & 37.7 & -84 & 17 & 15.5 & GPS \\
\hline 07228 & 38354 & 17243 & 35 & 55 & 36.7 & -84 & 17 & 14.6 & GPS \\
\hline 07230 & 38062 & 17537 & 35 & 55 & 37.5 & -84 & 17 & 19.5 & GPS \\
\hline 07235 & 38510 & 18029 & 35 & 55 & 44.0 & -84 & 17 & 18.3 & GPS \\
\hline 07240 & 38520 & 18089 & 35 & 55 & 44.6 & -84 & 17 & 18.6 & GPS \\
\hline 07245 & 38517 & 18223 & 35 & 55 & 45.7 & -84 & 17 & 19.6 & GPS \\
\hline 07250 & 38754 & 18514 & 35 & 55 & 49.4 & -84 & 17 & 19.2 & GPS \\
\hline 07255 & 38600 & 18897 & 35 & 55 & 51.7 & -84 & 17 & 23.3 & GPS \\
\hline 07260 & 38664 & 19265 & 35 & 55 & 55.0 & -84 & 17 & 25.1 & GPS \\
\hline 07300 & 38500 & 18227 & 35 & 55 & 45.6 & -84 & 17 & 19.8 & GPS \\
\hline 07345 & 38117 & 18798 & 35 & 55 & 48.2 & -84 & 17 & 27.5 & GPS \\
\hline 07350 & 38145 & 19378 & 35 & 55 & 53.1 & -84 & 17 & 31.1 & GPS \\
\hline 07353 & 37964 & 17933 & 35 & 55 & 40.2 & -84 & 17 & 23.2 & GPS \\
\hline 07355 & 38022 & 18172 & 35 & 55 & 42.5 & -84 & 17 & 24.2 & GPS \\
\hline 07360 & 37761 & 17904 & 35 & 55 & 38.9 & -84 & 17 & 25.1 & GPS \\
\hline 07362 & 37923 & 18215 & 35 & 55 & 42.3 & -84 & 17 & 25.5 & GPS \\
\hline 07365 & 37867 & 18560 & 35 & 55 & 44.9 & -84 & 17 & 28.4 & GPS \\
\hline 07370 & 37860 & 18253 & 35 & 55 & 42.3 & -84 & 17 & 26.4 & GPS \\
\hline 07375 & 37417 & 18233 & 35 & 55 & 39.7 & -84 & 17 & 30.8 & GPS \\
\hline 07380 & 37179 & 18322 & 35 & 55 & 39.1 & -84 & 17 & 33.8 & GPS \\
\hline 07385 & 37231 & 18084 & 35 & 55 & 37.5 & -84 & 17 & 31.6 & GPS \\
\hline 07395 & 36955 & 18274 & 35 & 55 & 37.5 & -84 & 17 & 35.7 & GPS \\
\hline 07400 & 37159 & 18434 & 35 & 55 & 39.9 & -84 & 17 & 34.7 & GPS \\
\hline 07405 & 36864 & 18742 & 35 & 55 & 40.8 & -84 & 17 & 39.8 & GPS \\
\hline 07410 & 36791 & 18808 & 35 & 55 & 41.0 & -84 & 17 & 41.0 & GPS \\
\hline 07415 & 37200 & 18498 & 35 & 55 & 40.7 & -84 & 17 & 34.8 & GPS \\
\hline 07420 & 37140 & 18904 & 35 & 55 & 43.7 & -84 & 17 & 38.1 & GPS \\
\hline 07425 & 37268 & 19357 & 35 & 55 & 48.1 & -84 & 17 & 39.9 & GPS \\
\hline 07430 & 37496 & 19601 & 35 & 55 & 51.4 & -84 & 17 & 39.2 & GPS \\
\hline 08005 & 29856 & 19812 & 35 & 55 & 11.1 & -84 & 18 & 57.8 & GPS \\
\hline 08010 & 30094 & 19780 & 35 & 55 & 12.1 & -84 & 18 & 55.2 & GPS \\
\hline 08013 & 30096 & 19598 & 35 & 55 & 10.7 & -84 & 18 & 54.0 & GPS \\
\hline 08015 & 30178 & 19666 & 35 & 55 & 11.7 & -84 & 18 & 53.6 & GPS \\
\hline 08020 & 30185 & 19431 & 35 & 55 & 9.8 & -84 & 18 & 51.9 & GPS \\
\hline 08025 & 30388 & 19640 & 35 & 55 & 12.6 & -84 & 18 & 51.3 & GPS \\
\hline 08028 & 30543 & 19438 & 35 & 55 & 11.8 & -84 & 18 & 48.4 & GPS \\
\hline 08030 & 30721 & 19329 & 35 & 55 & 11.9 & -84 & 18 & 45.8 & GPS \\
\hline 08031 & 30799 & 19448 & 35 & 55 & 13.3 & -84 & 18 & 45.9 & GPS \\
\hline 08033 & 30817 & 19458 & 35 & 55 & 13.5 & -84 & 18 & 45.7 & GPS \\
\hline 08034 & 30897 & 19434 & 35 & 55 & 13.7 & -84 & 18 & 44.8 & GPS \\
\hline 08035 & 30825 & 19529 & 35 & 55 & 14.1 & -84 & 18 & 46.1 & GPS \\
\hline 08037 & 31126 & 19060 & 35 & 55 & 11.9 & -84 & 18 & 39.9 & GPS \\
\hline
\end{tabular}


Table 2. Coordinates for seeps, springs, and stream-measurement sites at Oak Ridge National Laboratory--Continued

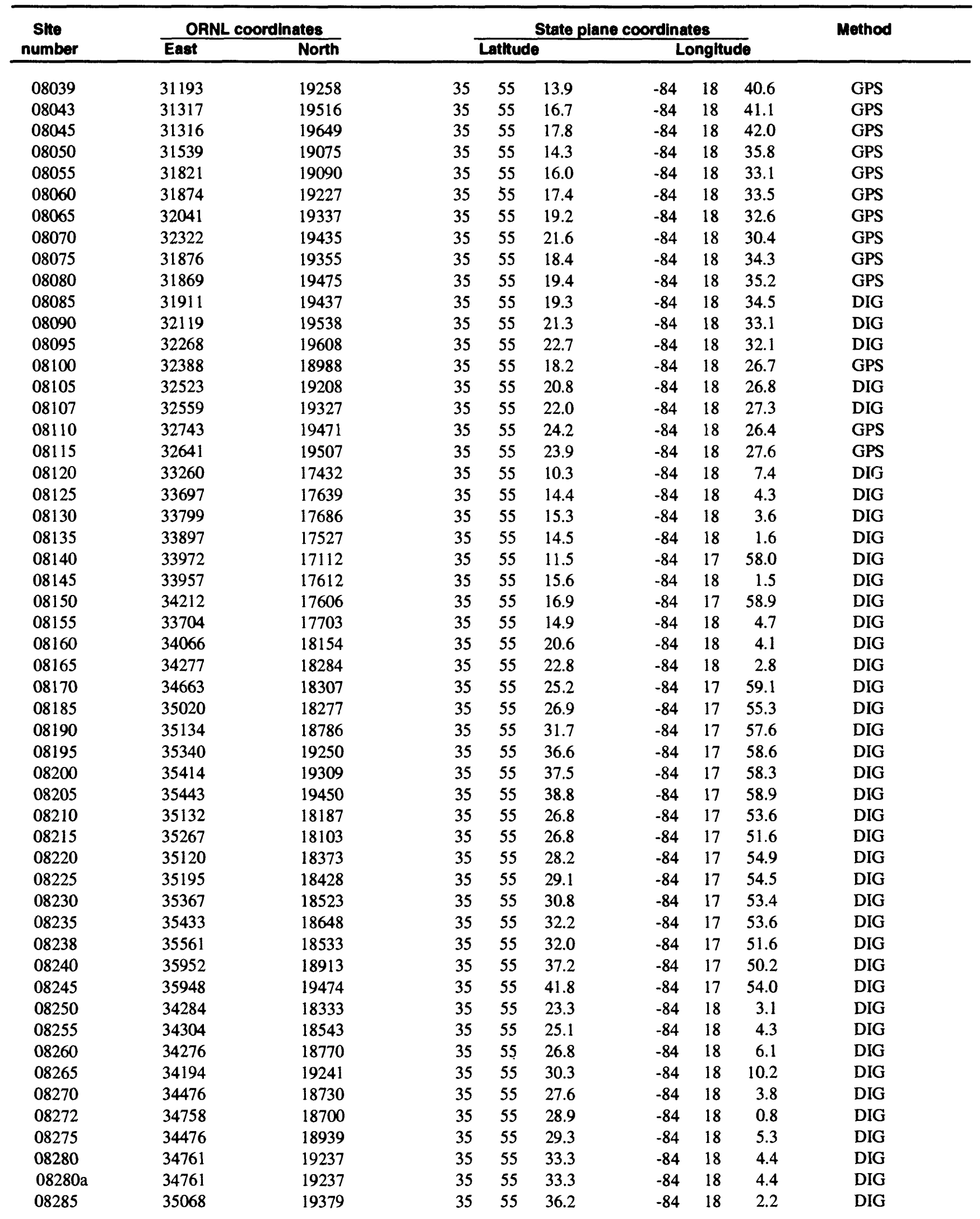


Table 2. Coordinates for seeps, springs, and stream-measurement sites at Oak Ridge National Laboratory--Continued

\begin{tabular}{|c|c|c|c|c|c|c|c|c|c|}
\hline \multirow{3}{*}{$\begin{array}{c}\begin{array}{c}\text { Site } \\
\text { number }\end{array} \\
08290\end{array}$} & \multicolumn{2}{|c|}{ ORNL coordinates } & \multicolumn{6}{|c|}{ State plane coordinates } & \multirow{3}{*}{$\frac{\text { Method }}{\text { DIG }}$} \\
\hline & \multirow{2}{*}{$\begin{array}{l}\text { East } \\
33284\end{array}$} & \multirow{2}{*}{$\frac{\text { North }}{17476}$} & \multicolumn{3}{|c|}{ Latitude } & \multicolumn{3}{|c|}{ Longitude } & \\
\hline & & & 35 & 55 & 10.8 & -84 & 18 & 7.4 & \\
\hline 08295 & 33630 & 17885 & 35 & 55 & 16.0 & -84 & 18 & 6.7 & DIG \\
\hline 08300 & 33860 & 18287 & 35 & 55 & 20.6 & -84 & 18 & 7.1 & DIG \\
\hline 08305 & 33852 & 18422 & 35 & 55 & 21.7 & -84 & 18 & 8.1 & DIG \\
\hline 08310 & 33209 & 17963 & 35 & 55 & 14.3 & -84 & 18 & 11.5 & DIG \\
\hline 08315 & 33297 & 18245 & 35 & 55 & 17.1 & -84 & 18 & 12.5 & DIG \\
\hline 08320 & 33402 & 18353 & 35 & 55 & 18.6 & -84 & 18 & 12.2 & DIG \\
\hline 08330 & 33462 & 18480 & 35 & 55 & 20.0 & -84 & 18 & 12.4 & DIG \\
\hline 08335 & 33415 & 18667 & 35 & 55 & 21.3 & -84 & 18 & 14.1 & DIG \\
\hline 08340 & 33757 & 18837 & 35 & 55 & 24.5 & -84 & 18 & 11.8 & DIG \\
\hline 08350 & 33288 & 18886 & 35 & 55 & 22.4 & -84 & 18 & 16.9 & DIG \\
\hline 08355 & 33548 & 18883 & 35 & 55 & 23.8 & -84 & 18 & 14.3 & DIG \\
\hline 08360 & 33804 & 19345 & 35 & 55 & 29.0 & -84 & 18 & 14.8 & DIG \\
\hline 08365 & 33727 & 19513 & 35 & 55 & 29.9 & -84 & 18 & 16.7 & DIG \\
\hline 08375 & 33214 & 18294 & 35 & 55 & 17.1 & -84 & 18 & 13.6 & DIG \\
\hline 08380 & 33041 & 18525 & 35 & 55 & 18.0 & -84 & 18 & 17.0 & DIG \\
\hline 08385 & 33058 & 18833 & 35 & 55 & 20.7 & -84 & 18 & 18.9 & DIG \\
\hline 08390 & 33245 & 19157 & 35 & 55 & 24.4 & -84 & 18 & 19.1 & DIG \\
\hline 08395 & 33173 & 19517 & 35 & 55 & 26.9 & -84 & 18 & 22.3 & GPS \\
\hline 08400 & 33165 & 19536 & 35 & 55 & 27.0 & -84 & 18 & 22.5 & GPS \\
\hline 09025 & 32645 & 16175 & 35 & 54 & 56.5 & -84 & 18 & 5.1 & GPS \\
\hline 09030 & 32853 & 15714 & 35 & 54 & 53.9 & -84 & 17 & 59.9 & DIG \\
\hline 09035 & 32647 & 15983 & 35 & 54 & 55.0 & -84 & 18 & 3.7 & GPS \\
\hline 09040 & 33338 & 15931 & 35 & 54 & 58.4 & -84 & 17 & 56.4 & GPS \\
\hline 09045 & 33400 & 15603 & 35 & 54 & 56.0 & -84 & 17 & 53.6 & GPS \\
\hline 09050 & 33249 & 15228 & 35 & 54 & 52.1 & -84 & 17 & 52.6 & GPS \\
\hline 09055 & 33165 & 15513 & 35 & 54 & 54.0 & -84 & 17 & 55.3 & GPS \\
\hline 09060 & 33794 & 15727 & 35 & 54 & 59.2 & -84 & 17 & 50.4 & GPS \\
\hline 09065 & 33636 & 15129 & 35 & 54 & 53.4 & -84 & 17 & 48.0 & GPS \\
\hline 09070 & 33936 & 15677 & 35 & 54 & 59.5 & -84 & 17 & 48.7 & GPS \\
\hline 09075 & 34026 & 15501 & 35 & 54 & 58.6 & -84 & 17 & 46.6 & GPS \\
\hline 09080 & 34011 & 15470 & 35 & 54 & 58.3 & -84 & 17 & 46.5 & GPS \\
\hline 09085 & 33878 & 14672 & 35 & 54 & 51.0 & -84 & 17 & 42.5 & GPS \\
\hline 09090 & 34213 & 15055 & 35 & 54 & 56.0 & -84 & 17 & 41.7 & GPS \\
\hline 09095 & 34191 & 14575 & 35 & 54 & 51.9 & -84 & 17 & 38.6 & GPS \\
\hline 09100 & 34601 & 15807 & 35 & 55 & 4.3 & -84 & 17 & 42.8 & GPS \\
\hline 09103 & 34987 & 15352 & 35 & 55 & 2.7 & -84 & 17 & 35.8 & GPS \\
\hline 09105 & 34768 & 15378 & 35 & 55 & 1.7 & -84 & 17 & 38.2 & GPS \\
\hline 09110 & 34711 & 15079 & 35 & 54 & 58.9 & -84 & 17 & 36.8 & GPS \\
\hline 09115 & 34994 & 14946 & 35 & 54 & 59.4 & -84 & 17 & 33.0 & GPS \\
\hline 09125 & 35249 & 15676 & 35 & 55 & 6.8 & -84 & 17 & 35.4 & DIG \\
\hline 09130 & 35479 & 15270 & 35 & 55 & 4.7 & -84 & 17 & 30.3 & DIG \\
\hline 09135 & 35635 & 14898 & 35 & 55 & 2.5 & -84 & 17 & 26.2 & DIG \\
\hline 09140 & 35906 & 14560 & 35 & 55 & 1.2 & -84 & 17 & 21.2 & DIG \\
\hline 09375 & 36133 & 15100 & 35 & 55 & 6.9 & -84 & 17 & 22.6 & DIG \\
\hline 09385 & 36291 & 14702 & 35 & 55 & 4.5 & -84 & 17 & 18.3 & DIG \\
\hline 09390 & 36455 & 14397 & 35 & 55 & 2.9 & -84 & 17 & 14.6 & DIG \\
\hline 09395 & 36798 & 14122 & 35 & 55 & 2.5 & -84 & 17 & 9.2 & DIG \\
\hline 09400 & 36780 & 13580 & 35 & 54 & 58.0 & -84 & 17 & 5.8 & DIG \\
\hline 10005 & 26822 & 16355 & 35 & 54 & 26.0 & -84 & 19 & 5.1 & GPS \\
\hline 10010 & 26941 & 16002 & 35 & 54 & 23.7 & -84 & 19 & 1.5 & DIG \\
\hline 10015 & 27120 & 15773 & 35 & 54 & 22.9 & -84 & 18 & 58.2 & GPS \\
\hline 10020 & 27430 & 15025 & 35 & 54 & 18.4 & -84 & 18 & 50.0 & GPS \\
\hline
\end{tabular}


Table 2. Coordinates for seeps, springs, and stream-measurement sites at Oak Ridge National Laboratory--Continued

\begin{tabular}{|c|c|c|c|c|c|c|c|c|c|}
\hline \multirow{3}{*}{$\begin{array}{c}\begin{array}{c}\text { Site } \\
\text { number }\end{array} \\
10170\end{array}$} & \multicolumn{2}{|c|}{ ORNL coordinates } & \multicolumn{6}{|c|}{ State plane coordinates } & \multirow{3}{*}{$\begin{array}{c}\text { Method } \\
\text { GPS }\end{array}$} \\
\hline & \multirow{2}{*}{$\begin{array}{l}\text { East } \\
27244\end{array}$} & \multirow{2}{*}{$\frac{\text { North }}{16393}$} & \multicolumn{3}{|c|}{ Latlude } & \multicolumn{3}{|c|}{ Longltude } & \\
\hline & & & 35 & 54 & 28.6 & -84 & 19 & 1.1 & \\
\hline 10175 & 27581 & 15812 & 35 & 54 & 25.7 & -84 & 18 & 53.8 & GPS \\
\hline 10180 & 27383 & 16062 & 35 & 54 & 26.7 & -84 & 18 & 57.5 & GPS \\
\hline 10185 & 27412 & 15725 & 35 & 54 & 24.1 & -84 & 18 & 54.9 & GPS \\
\hline 10189 & 27521 & 15548 & 35 & 54 & 23.2 & -84 & 18 & 52.6 & DIG \\
\hline 10190 & 27711 & 15478 & 35 & 54 & 23.7 & -84 & 18 & 50.2 & GPS \\
\hline 10195 & 27765 & 15482 & 35 & 54 & 24.0 & -84 & 18 & 49.7 & GPS \\
\hline 10200 & 27948 & 15323 & 35 & 54 & 23.7 & -84 & 18 & 46.8 & GPS \\
\hline 10205 & 27853 & 16664 & 35 & 54 & 34.2 & -84 & 18 & 56.8 & GPS \\
\hline 10220 & 27937 & 16302 & 35 & 54 & 31.7 & -84 & 18 & 53.5 & GPS \\
\hline 10225 & 28164 & 16020 & 35 & 54 & 30.6 & -84 & 18 & 49.3 & GPS \\
\hline 10230 & 28214 & 16072 & 35 & 54 & 31.3 & -84 & 18 & 49.1 & GPS \\
\hline 10235 & 28361 & 15789 & 35 & 54 & 29.8 & -84 & 18 & 45.7 & GPS \\
\hline 10240 & 28441 & 15879 & 35 & 54 & 31.0 & -84 & 18 & 45.5 & GPS \\
\hline 10325 & 28658 & 16548 & 35 & 54 & 37.7 & -84 & 18 & 47.9 & DIG \\
\hline 10335 & 28534 & 16279 & 35 & 54 & 34.8 & -84 & 18 & 47.3 & DIG \\
\hline 10340 & 28541 & 16102 & 35 & 54 & 33.4 & -84 & 18 & 46.0 & GPS \\
\hline 10350 & 28713 & 15835 & 35 & 54 & 32.1 & -84 & 18 & 42.5 & GPS \\
\hline 10355 & 28804 & 16318 & 35 & 54 & 36.6 & -84 & 18 & 44.8 & DIG \\
\hline 10360 & 28845 & 15827 & 35 & 54 & 32.8 & -84 & 18 & 41.1 & DIG \\
\hline 10365 & 29550 & 16600 & 35 & 54 & 43.0 & -84 & 18 & 39.2 & DIG \\
\hline 10370 & 29422 & 16240 & 35 & 54 & 39.4 & -84 & 18 & 38.1 & DIG \\
\hline 10390 & 29518 & 16417 & 35 & 54 & 41.3 & -84 & 18 & 38.3 & DIG \\
\hline 10395 & 29771 & 15873 & 35 & 54 & 38.3 & -84 & 18 & 32.1 & DIG \\
\hline 10400 & 29832 & 16581 & 35 & 54 & 44.4 & -84 & 18 & 36.2 & GPS \\
\hline 10405 & 29953 & 16340 & 35 & 54 & 43.1 & -84 & 18 & 33.4 & GPS \\
\hline 10410 & 30063 & 15979 & 35 & 54 & 40.7 & -84 & 18 & 29.8 & GPS \\
\hline 10430 & 30541 & 16564 & 35 & 54 & 48.2 & -84 & 18 & 29.0 & GPS \\
\hline 10450 & 30374 & 16339 & 35 & 54 & 45.4 & -84 & 18 & 29.1 & GPS \\
\hline 10460 & 31023 & 16382 & 35 & 54 & 49.3 & -84 & 18 & 22.9 & GPS \\
\hline 10470 & 30877 & 15849 & 35 & 54 & 44.1 & -84 & 18 & 20.7 & GPS \\
\hline 10475 & 31280 & 16279 & 35 & 54 & 49.9 & -84 & 18 & 19.6 & GPS \\
\hline 10480 & 31225 & 15932 & 35 & 54 & 46.7 & -84 & 18 & 17.8 & GPS \\
\hline 10485 & 31589 & 16195 & 35 & 54 & 50.9 & -84 & 18 & 15.9 & GPS \\
\hline 10490 & 31630 & 16048 & 35 & 54 & 49.9 & -84 & 18 & 14.5 & GPS \\
\hline 10510 & 31551 & 15360 & 35 & 54 & 43.8 & -84 & 18 & 10.6 & GPS \\
\hline 10600 & 32143 & 16097 & 35 & 54 & 53.1 & -84 & 18 & 9.6 & GPS \\
\hline 10605 & 31923 & 15525 & 35 & 54 & 47.2 & -84 & 18 & 8.0 & GPS \\
\hline 10607 & 31974 & 15323 & 35 & 54 & 45.8 & -84 & 18 & 6.1 & GPS \\
\hline 10610 & 31982 & 15159 & 35 & 54 & 44.6 & -84 & 18 & 4.9 & GPS \\
\hline 11005 & 23200 & 14275 & 35 & 53 & 49.0 & -84 & 19 & 27.6 & GPS \\
\hline 11010 & 23511 & 14139 & 35 & 53 & 49.6 & -84 & 19 & 23.6 & DIG \\
\hline 11015 & 23388 & 14290 & 35 & 53 & 50.1 & -84 & 19 & 25.8 & DIG \\
\hline 11020 & 23256 & 14394 & 35 & 53 & 50.3 & -84 & 19 & 27.9 & GPS \\
\hline 11025 & 23679 & 14302 & 35 & 53 & 51.8 & -84 & 19 & 23.0 & DIG \\
\hline 11030 & 23278 & 14476 & 35 & 53 & 51.1 & -84 & 19 & 28.2 & GPS \\
\hline 11035 & 23455 & 14510 & 35 & 53 & 52.3 & -84 & 19 & 26.6 & DIG \\
\hline 11040 & 23824 & 15178 & 35 & 53 & 59.8 & -84 & 19 & 27.4 & DIG \\
\hline 11045 & 23812 & 14998 & 35 & 53 & 58.3 & -84 & 19 & 26.3 & DIG \\
\hline 11050 & 24284 & 15146 & 35 & 54 & 2.1 & -84 & 19 & 22.6 & DIG \\
\hline 11055 & 24284 & 14922 & 35 & 54 & 0.3 & -84 & 19 & 21.1 & DIG \\
\hline 11060 & 24756 & 15264 & 35 & 54 & 5.7 & -84 & 19 & 18.6 & DIG \\
\hline 11070 & 24843 & 14816 & 35 & 54 & 2.5 & -84 & 19 & 14.7 & DIG \\
\hline
\end{tabular}


Table 2. Coordinates for seeps, springs, and stream-measurement sites at Oak Ridge National Laboratory--Continued

\begin{tabular}{|c|c|c|c|c|c|c|c|c|c|}
\hline \multirow{3}{*}{$\begin{array}{c}\begin{array}{c}\text { Site } \\
\text { number }\end{array} \\
11080\end{array}$} & \multicolumn{2}{|c|}{ ORNL coordinates } & \multicolumn{6}{|c|}{ State plane coordinates } & \multirow{3}{*}{ Method } \\
\hline & \multirow{2}{*}{$\begin{array}{l}\text { East } \\
25617\end{array}$} & \multirow{2}{*}{$\begin{array}{l}\text { North } \\
15880\end{array}$} & \multicolumn{3}{|c|}{ Latitude } & \multicolumn{3}{|c|}{ Longitude } & \\
\hline & & & 35 & 54 & 15.5 & -84 & 19 & 14.1 & \\
\hline 11082 & 25659 & 15755 & 35 & 54 & 14.7 & -84 & 19 & 12.8 & DIG \\
\hline 11085 & 26029 & 16197 & 35 & 54 & 20.3 & -84 & 19 & 12.1 & DIG \\
\hline 11087 & 26389 & 16288 & 35 & 54 & 23.1 & -84 & 19 & 9.0 & DIG \\
\hline 11089 & 26436 & 16110 & 35 & 54 & 21.9 & -84 & 19 & 7.4 & DIG \\
\hline 11093 & 26465 & 15710 & 35 & 54 & 18.7 & -84 & 19 & 4.4 & DIG \\
\hline 11095 & 26649 & 15274 & 35 & 54 & 16.2 & -84 & 18 & 59.6 & DIG \\
\hline 11100 & 26732 & 14829 & 35 & 54 & 13.0 & -84 & 18 & 55.7 & DIG \\
\hline 11105 & 26678 & 15293 & 35 & 54 & 16.5 & -84 & 18 & 59.4 & DIG \\
\hline 11110 & 26742 & 15188 & 35 & 54 & 16.0 & -84 & 18 & 58.0 & DIG \\
\hline 12010 & 21564 & 18020 & 35 & 54 & 10.8 & -84 & 20 & 9.5 & GPS \\
\hline 12015 & 21570 & 17595 & 35 & 54 & 7.3 & -84 & 20 & 6.5 & GPS \\
\hline 12020 & 22179 & 17580 & 35 & 54 & 10.5 & -84 & 20 & 0.3 & GPS \\
\hline 12025 & 22343 & 17762 & 35 & 54 & 12.9 & -84 & 19 & 59.9 & GPS \\
\hline 12040 & 22311 & 15996 & 35 & 53 & 58.2 & -84 & 19 & 48.3 & DIG \\
\hline 12045 & 22496 & 16431 & 35 & 54 & 2.8 & -84 & 19 & 49.3 & DIG \\
\hline 12090 & 21890 & 16635 & 35 & 54 & 1.2 & -84 & 19 & 56.8 & DIG \\
\hline 12095 & 21697 & 16770 & 35 & 54 & 1.2 & -84 & 19 & 59.7 & DIG \\
\hline 12100 & 21642 & 17274 & 35 & 54 & 5.1 & -84 & 20 & 3.6 & DIG \\
\hline 12105 & 22438 & 17280 & 35 & 54 & 9.5 & -84 & 19 & 55.7 & DIG \\
\hline 12110 & 22543 & 17269 & 35 & 54 & 10.0 & -84 & 19 & 54.5 & DIG \\
\hline 12115 & 22759 & 17160 & 35 & 54 & 10.3 & -84 & 19 & 51.6 & DIG \\
\hline 12120 & 22806 & 17468 & 35 & 54 & 13.1 & -84 & 19 & 53.2 & DIG \\
\hline 12125 & 22878 & 17481 & 35 & 54 & 13.6 & -84 & 19 & 52.6 & DIG \\
\hline 12130 & 23164 & 17794 & 35 & 54 & 17.7 & -84 & 19 & 51.8 & DIG \\
\hline 12132 & 23271 & 17809 & 35 & 54 & 18.4 & -84 & 19 & 50.8 & DIG \\
\hline 12135 & 23693 & 17793 & 35 & 54 & 20.6 & -84 & 19 & 46.4 & DIG \\
\hline 12140 & 23464 & 18195 & 35 & 54 & 22.7 & -84 & 19 & 51.5 & DIG \\
\hline 12145 & 23494 & 18131 & 35 & 54 & 22.3 & -84 & 19 & 50.7 & DIG \\
\hline 12148 & 23722 & 18160 & 35 & 54 & 23.8 & -84 & 19 & 48.6 & DIG \\
\hline 12150 & 24076 & 17908 & 35 & 54 & 23.7 & -84 & 19 & 43.4 & DIG \\
\hline 12155 & 23951 & 18328 & 35 & 54 & 26.4 & -84 & 19 & 47.5 & DIG \\
\hline 12160 & 24283 & 18147 & 35 & 54 & 26.8 & -84 & 19 & 42.9 & DIG \\
\hline 13140 & 20908 & 18950 & 35 & 54 & 14.8 & -84 & 20 & 22.4 & DIG \\
\hline 13150 & 20963 & 19082 & 35 & 54 & 16.2 & -84 & 20 & 22.7 & DIG \\
\hline 13155 & 21147 & 19196 & 35 & 54 & 18.1 & -84 & 20 & 21.6 & DIG \\
\hline 13160 & 21223 & 19306 & 35 & 54 & 19.4 & -84 & 20 & 21.6 & DIG \\
\hline 13162 & 21239 & 19256 & 35 & 54 & 19.1 & -84 & 20 & 21.1 & DIG \\
\hline 13165 & 21421 & 19439 & 35 & 54 & 21.6 & -84 & 20 & 20.5 & DIG \\
\hline 13170 & 21399 & 19487 & 35 & 54 & 21.9 & -84 & 20 & 21.1 & DIG \\
\hline 13175 & 21098 & 18649 & 35 & 54 & 13.4 & -84 & 20 & 18.4 & DIG \\
\hline 13180 & 21317 & 18391 & 35 & 54 & 12.4 & -84 & 20 & 14.5 & DIG \\
\hline 13185 & 21262 & 18431 & 35 & 54 & 12.5 & -84 & 20 & 15.3 & DIG \\
\hline 13190 & 21614 & 18581 & 35 & 54 & 15.6 & -84 & 20 & 12.8 & DIG \\
\hline 13195 & 21792 & 18650 & 35 & 54 & 17.2 & -84 & 20 & 11.4 & DIG \\
\hline 13200 & 21808 & 18718 & 35 & 54 & 17.8 & -84 & 20 & 11.7 & GPS \\
\hline 13205 & 21867 & 18754 & 35 & 54 & 18.5 & -84 & 20 & 11.4 & GPS \\
\hline 13210 & 22047 & 18995 & 35 & 54 & 21.4 & -84 & 20 & 11.2 & GPS \\
\hline 13215 & 22124 & 19005 & 35 & 54 & 21.9 & -84 & 20 & 10.5 & GPS \\
\hline 13220 & 22585 & 19095 & 35 & 54 & 25.2 & -84 & 20 & 6.4 & GPS \\
\hline 13225 & 22707 & 19439 & 35 & 54 & 28.7 & -84 & 20 & 7.5 & GPS \\
\hline 13230 & 22215 & 19520 & 35 & 54 & 26.7 & -84 & 20 & 13.1 & GPS \\
\hline 13235 & 22307 & 19420 & 35 & 54 & 26.3 & -84 & 20 & 11.5 & DIG \\
\hline
\end{tabular}


Table 2. Coordinates for seeps, springs, and stream-measurement sites at Oak Ridge National Laboratory--Continued

\begin{tabular}{|c|c|c|c|c|c|c|c|c|c|}
\hline \multirow{3}{*}{$\begin{array}{c}\begin{array}{c}\text { Stte } \\
\text { number }\end{array} \\
13250\end{array}$} & \multicolumn{2}{|c|}{ ORNL coordinates } & \multicolumn{6}{|c|}{ State plane coordinates } & \multirow{3}{*}{$\begin{array}{c}\text { Method } \\
\text { GPS }\end{array}$} \\
\hline & \multirow{2}{*}{$\begin{array}{l}\text { East } \\
22351\end{array}$} & \multirow{2}{*}{$\frac{\text { North }}{18664}$} & \multicolumn{3}{|c|}{ Latitude } & \multicolumn{3}{|c|}{ Longltude } & \\
\hline & & & 35 & 54 & 20.4 & -84 & 20 & 5.9 & \\
\hline 13255 & 23066 & 18768 & 35 & 54 & 25.2 & -84 & 19 & 59.4 & GPS \\
\hline 13260 & 22800 & 18765 & 35 & 54 & 23.7 & -84 & 20 & 2.0 & GPS \\
\hline 13265 & 22908 & 18887 & 35 & 54 & 25.3 & -84 & 20 & 1.8 & GPS \\
\hline 13270 & 22918 & 18771 & 35 & 54 & 24.4 & -84 & 20 & 0.9 & GPS \\
\hline 13274 & 23174 & 18950 & 35 & 54 & 27.3 & -84 & 19 & 59.5 & GPS \\
\hline 13275 & 23200 & 19007 & 35 & 54 & 27.9 & -84 & 19 & 59.6 & GPS \\
\hline 13280 & 23263 & 19342 & 35 & 54 & 31.0 & -84 & 20 & 1.3 & GPS \\
\hline 13285 & 23530 & 19253 & 35 & 54 & 31.7 & -84 & 19 & 58.0 & GPS \\
\hline 13290 & 23526 & 19730 & 35 & 54 & 35.6 & -84 & 20 & 1.2 & GPS \\
\hline 13295 & 23508 & 19838 & 35 & 54 & 36.4 & -84 & 20 & 2.1 & GPS \\
\hline 13300 & 22944 & 18770 & 35 & 54 & 24.5 & -84 & 20 & 0.6 & GPS \\
\hline 13305 & 23348 & 18820 & 35 & 54 & 27.1 & -84 & 19 & 56.9 & DIG \\
\hline 13310 & 23363 & 18746 & 35 & 54 & 26.6 & -84 & 19 & 56.2 & GPS \\
\hline 13315 & 23649 & 18798 & 35 & 54 & 28.6 & -84 & 19 & 53.7 & GPS \\
\hline 13320 & 23710 & 18942 & 35 & 54 & 30.1 & -84 & 19 & 54.0 & GPS \\
\hline 13326 & 23928 & 18830 & 35 & 54 & 30.4 & -84 & 19 & 51.1 & DIG \\
\hline 13328 & 24255 & 18760 & 35 & 54 & 31.6 & -84 & 19 & 47.3 & DIG \\
\hline 13330 & 23913 & 18902 & 35 & 54 & 30.9 & -84 & 19 & 51.7 & DIG \\
\hline 13335 & 23972 & 18945 & 35 & 54 & 31.6 & -84 & 19 & 51.4 & DIG \\
\hline 13340 & 24174 & 18964 & 35 & 54 & 32.9 & -84 & 19 & 49.5 & DIG \\
\hline 13345 & 24098 & 19242 & 35 & 54 & 34.7 & -84 & 19 & 52.2 & DIG \\
\hline 13350 & 23905 & 19554 & 35 & 54 & 36.2 & -84 & 19 & 56.2 & DIG \\
\hline 13355 & 24162 & 19255 & 35 & 54 & 35.2 & -84 & 19 & 51.6 & DIG \\
\hline 13358 & 24338 & 19430 & 35 & 54 & 37.6 & -84 & 19 & 51.0 & DIG \\
\hline 13360 & 24372 & 19670 & 35 & 54 & 39.8 & -84 & 19 & 52.3 & DIG \\
\hline 13365 & 24330 & 19697 & 35 & 54 & 39.8 & -84 & 19 & 52.9 & DIG \\
\hline 13370 & 24251 & 19868 & 35 & 54 & 40.7 & -84 & 19 & 54.8 & DIG \\
\hline 14005 & 24924 & 19077 & 35 & 54 & 37.9 & -84 & 19 & 42.7 & GPS \\
\hline 14010 & 24958 & 19106 & 35 & 54 & 38.4 & -84 & 19 & 42.5 & GPS \\
\hline 14015 & 24982 & 19167 & 35 & 54 & 39.0 & -84 & 19 & 42.7 & GPS \\
\hline 14025 & 24860 & 19412 & 35 & 54 & 40.3 & -84 & 19 & 45.6 & GPS \\
\hline 14060 & 25054 & 19537 & 35 & 54 & 42.4 & -84 & 19 & 44.5 & GPS \\
\hline 14070 & 25238 & 19189 & 35 & 54 & 40.6 & -84 & 19 & 40.3 & DIG \\
\hline 14075 & 25019 & 19088 & 35 & 54 & 38.5 & -84 & 19 & 41.8 & GPS \\
\hline 14080 & 25487 & 19230 & 35 & 54 & 42.3 & -84 & 19 & 38.0 & DIG \\
\hline 14095 & 25256 & 19442 & 35 & 54 & 42.8 & -84 & 19 & 41.8 & DIG \\
\hline 14100 & 25238 & 19629 & 35 & 54 & 44.2 & -84 & 19 & 43.3 & DIG \\
\hline 14110 & 25604 & 19256 & 35 & 54 & 43.1 & -84 & 19 & 37.0 & GPS \\
\hline 14130 & 25480 & 19926 & 35 & 54 & 48.0 & -84 & 19 & 42.8 & GPS \\
\hline 14135 & 26080 & 19191 & 35 & 54 & 45.2 & -84 & 19 & 31.8 & DIG \\
\hline 14140 & 26026 & 19332 & 35 & 54 & 46.1 & -84 & 19 & 33.3 & DIG \\
\hline 14145 & 26028 & 19447 & 35 & 54 & 47.0 & -84 & 19 & 34.0 & DIG \\
\hline 14160 & 26230 & 19561 & 35 & 54 & 49.1 & -84 & 19 & 32.8 & DIG \\
\hline 14175 & 25992 & 19791 & 35 & 54 & 49.7 & -84 & 19 & 36.7 & DIG \\
\hline 14180 & 26013 & 19928 & 35 & 54 & 50.9 & -84 & 19 & 37.5 & DIG \\
\hline 14190 & 25830 & 20081 & 35 & 54 & 51.2 & -84 & 19 & 40.3 & GPS \\
\hline 14280 & 26455 & 19171 & 35 & 54 & 47.1 & -84 & 19 & 27.9 & DIG \\
\hline 14285 & 26400 & 19347 & 35 & 54 & 48.3 & -84 & 19 & 29.6 & DIG \\
\hline 14290 & 26616 & 19537 & 35 & 54 & 51.0 & -84 & 19 & 28.7 & DIG \\
\hline 14295 & 26401 & 19400 & 35 & 54 & 48.7 & -84 & 19 & 30.0 & DIG \\
\hline 14297 & 26501 & 19860 & 35 & 54 & 53.0 & -84 & 19 & 32.1 & DIG \\
\hline 14305 & 26819 & 19192 & 35 & 54 & 49.3 & .84 & 19 & 24.3 & DIG \\
\hline
\end{tabular}


Table 2. Coordinates for seeps, springs, and stream-measurement sites at Oak Ridge National Laboratory--Continued

\begin{tabular}{|c|c|c|c|c|c|c|c|c|c|}
\hline \multirow{3}{*}{$\begin{array}{c}\begin{array}{c}\text { Site } \\
\text { number }\end{array} \\
14310\end{array}$} & \multicolumn{2}{|c|}{ ORNL coordinates } & \multicolumn{6}{|c|}{ State plane coordinates } & \multirow{3}{*}{$\begin{array}{c}\text { Method } \\
\text { DIG }\end{array}$} \\
\hline & \multirow{2}{*}{$\begin{array}{l}\text { East } \\
26894\end{array}$} & \multirow{2}{*}{$\frac{\text { North }}{19475}$} & \multicolumn{3}{|c|}{ Latitude } & \multicolumn{3}{|c|}{ Longitude } & \\
\hline & & & 35 & 54 & 52.0 & -84 & 19 & 25.5 & \\
\hline 14315 & 26923 & 19717 & 35 & 54 & 54.2 & -84 & 19 & 26.8 & DIG \\
\hline 14320 & 26997 & 19789 & 35 & 54 & 55.2 & -84 & 19 & 26.6 & DIG \\
\hline 14325 & 27075 & 19870 & 35 & 54 & 56.3 & -84 & 19 & 26.3 & DIG \\
\hline 14330 & 27033 & 20255 & 35 & 54 & 59.2 & -84 & 19 & 29.4 & DIG \\
\hline 14335 & 27014 & 20414 & 35 & 55 & 0.4 & -84 & 19 & 30.6 & DIG \\
\hline 14340 & 27166 & 20567 & 35 & 55 & 2.5 & -84 & 19 & 30.1 & DIG \\
\hline 14345 & 27196 & 19390 & 35 & 54 & 53.0 & -84 & 19 & 21.9 & DIG \\
\hline 14350 & 27307 & 19582 & 35 & 54 & 55.2 & -84 & 19 & 22.0 & DIG \\
\hline 14355 & 27477 & 19520 & 35 & 54 & 55.6 & -84 & 19 & 19.9 & DIG \\
\hline 14360 & 27496 & 19762 & 35 & 54 & 57.7 & -84 & 19 & 21.3 & DIG \\
\hline 14365 & 27499 & 19924 & 35 & 54 & 59.1 & -84 & 19 & 22.4 & DIG \\
\hline 14370 & 27418 & 20069 & 35 & 54 & 59.8 & -84 & 19 & 24.2 & DIG \\
\hline 14375 & 27842 & 20054 & 35 & 55 & 2.0 & -84 & 19 & 19.8 & DIG \\
\hline 14380 & 27888 & 20173 & 35 & 55 & 3.2 & -84 & 19 & 20.2 & DIG \\
\hline 14685 & 28293 & 19527 & 35 & 55 & 0.2 & -84 & 19 & 11.7 & DIG \\
\hline 14690 & 28291 & 19640 & 35 & 55 & 1.1 & -84 & 19 & 12.5 & DIG \\
\hline 14695 & 28413 & 20076 & 35 & 55 & 5.3 & -84 & 19 & 14.2 & DIG \\
\hline 14700 & 28288 & 20092 & 35 & 55 & 4.8 & -84 & 19 & 15.6 & DIG \\
\hline 14705 & 28494 & 19633 & 35 & 55 & 2.1 & -84 & 19 & 10.4 & DIG \\
\hline 14710 & 28654 & 19881 & 35 & 55 & 5.1 & -84 & 19 & 10.4 & DIG \\
\hline 14715 & 29093 & 19671 & 35 & 55 & 5.7 & -84 & 19 & 4.6 & DIG \\
\hline 14720 & 29047 & 19878 & 35 & 55 & 7.2 & -84 & 19 & 6.5 & DIG \\
\hline 14725 & 28981 & 20155 & 35 & 55 & 9.1 & -84 & 19 & 9.0 & DIG \\
\hline 14870 & 29360 & 19892 & 35 & 55 & 9.0 & -84 & 19 & 3.4 & DIG \\
\hline 14875 & 29256 & 20121 & 35 & 55 & 10.3 & -84 & 19 & 6.0 & DIG \\
\hline 15004 & 22500 & 14742 & 35 & 53 & 49.0 & -84 & 19 & 37.9 & GPS \\
\hline 15005 & 22536 & 14507 & 35 & 53 & 47.2 & -84 & 19 & 35.9 & GPS \\
\hline 15010 & 22574 & 14775 & 35 & 53 & 49.6 & -84 & 19 & 37.3 & GPS \\
\hline 15015 & 22581 & 14593 & 35 & 53 & 48.2 & -84 & 19 & 36.0 & GPS \\
\hline 15025 & 22877 & 14864 & 35 & 53 & 52.1 & -84 & 19 & 34.9 & GPS \\
\hline 15030 & 23009 & 14775 & 35 & 53 & 52.0 & -84 & 19 & 32.9 & DIG \\
\hline 15035 & 23080 & 14570 & 35 & 53 & 50.7 & -84 & 19 & 30.8 & GPS \\
\hline 15038 & 23134 & 14509 & 35 & 53 & 50.5 & -84 & 19 & 29.9 & GPS \\
\hline 15045 & 23095 & 14432 & 35 & 53 & 49.7 & -84 & 19 & 29.8 & GPS \\
\hline 15050 & 23144 & 14236 & 35 & 53 & 48.4 & -84 & 19 & 27.9 & GPS \\
\hline 15055 & 23078 & 14410 & 35 & 53 & 49.4 & -84 & 19 & 29.8 & GPS \\
\hline 15060 & 23068 & 14309 & 35 & 53 & 48.5 & -84 & 19 & 29.2 & GPS \\
\hline 15065 & 21370 & 14128 & 35 & 53 & 37.7 & -84 & 19 & 45.1 & GPS \\
\hline 15070 & 21588 & 14045 & 35 & 53 & 38.2 & -84 & 19 & 42.4 & DIG \\
\hline 15080 & 22054 & 14418 & 35 & 53 & 43.9 & -84 & 19 & 40.2 & DIG \\
\hline 16005 & 30349 & 20635 & 35 & 55 & 20.6 & -84 & 18 & 58.4 & DIG \\
\hline 16010 & 30522 & 20487 & 35 & 55 & 20.3 & -84 & 18 & 55.7 & DIG \\
\hline 16015 & 30641 & 20320 & 35 & 55 & 19.6 & -84 & 18 & 53.3 & DIG \\
\hline 16020 & 30871 & 20964 & 35 & 55 & 26.2 & -84 & 18 & 55.4 & DIG \\
\hline 16023 & 31215 & 20734 & 35 & 55 & 26.1 & -84 & 18 & 50.3 & DIG \\
\hline 16025 & 31197 & 20698 & 35 & 55 & 25.8 & -84 & 18 & 50.3 & DIG \\
\hline 16030 & 31446 & 20302 & 35 & 55 & 23.9 & -84 & 18 & 45.1 & DIG \\
\hline 16035 & 32787 & 21260 & 35 & 55 & 39.1 & -84 & 18 & 38.0 & GPS \\
\hline 16040 & 32775 & 21032 & 35 & 55 & 37.2 & -84 & 18 & 36.6 & GPS \\
\hline 16045 & 32775 & 20986 & 35 & 55 & 36.8 & -84 & 18 & 36.3 & GPS \\
\hline 16050 & 32787 & 20809 & 35 & 55 & 35.4 & -84 & 18 & 35.0 & GPS \\
\hline 16055 & 32719 & 20671 & 35 & 55 & 33.9 & -84 & 18 & 34.7 & GPS \\
\hline
\end{tabular}


Table 2. Coordinates for seeps, springs, and stream-measurement sites at Oak Ridge National Laboratory--Continued

\begin{tabular}{|c|c|c|c|c|c|c|c|c|c|}
\hline \multirow{3}{*}{$\begin{array}{c}\begin{array}{c}\text { Site } \\
\text { number }\end{array} \\
16060\end{array}$} & \multicolumn{2}{|c|}{ ORNL coordinates } & \multicolumn{6}{|c|}{ State plane coordinates } & \multirow{3}{*}{$\begin{array}{c}\text { Method } \\
\text { GPS }\end{array}$} \\
\hline & \multirow{2}{*}{$\begin{array}{c}\text { East } \\
32726\end{array}$} & \multirow{2}{*}{$\frac{\text { North }}{20766}$} & \multicolumn{3}{|c|}{ Latitude } & \multicolumn{3}{|c|}{ Longltude } & \\
\hline & & & 35 & 55 & 34.7 & -84 & 18 & 35.3 & \\
\hline 16065 & 32732 & 20630 & 35 & 55 & 33.6 & -84 & 18 & 34.3 & GPS \\
\hline 16075 & 32687 & 20437 & 35 & 55 & 31.8 & -84 & 18 & 33.5 & GPS \\
\hline 16080 & 32671 & 20106 & 35 & 55 & 29.0 & -84 & 18 & 31.4 & GPS \\
\hline 16085 & 32684 & 20079 & 35 & 55 & 28.8 & -84 & 18 & 31.1 & GPS \\
\hline 16090 & 33468 & 21061 & 35 & 55 & 41.2 & -84 & 18 & 29.8 & GPS \\
\hline 16095 & 33621 & 20928 & 35 & 55 & 41.0 & -84 & 18 & 27.3 & GPS \\
\hline 16100 & 33617 & 20900 & 35 & 55 & 40.7 & -84 & 18 & 27.2 & GPS \\
\hline 16105 & 34041 & 20886 & 35 & 55 & 42.9 & -84 & 18 & 22.8 & GPS \\
\hline 16110 & 34184 & 20715 & 35 & 55 & 42.3 & -84 & 18 & 20.2 & GPS \\
\hline 16115 & 33901 & 20426 & 35 & 55 & 38.4 & -84 & 18 & 21.1 & GPS \\
\hline 16120 & 34209 & 20630 & 35 & 55 & 41.8 & -84 & 18 & 19.4 & GPS \\
\hline 16125 & 34152 & 20492 & 35 & 55 & 40.3 & -84 & 18 & 19.0 & GPS \\
\hline 16130 & 34160 & 20503 & 35 & 55 & 40.4 & -84 & 18 & 19.0 & GPS \\
\hline 16135 & 34188 & 19946 & 35 & 55 & 36.0 & -84 & 18 & 15.0 & GPS \\
\hline 16140 & 33568 & 21564 & 35 & 55 & 45.9 & -84 & 18 & 32.2 & GPS \\
\hline 16145 & 33598 & 21592 & 35 & 55 & 46.3 & -84 & 18 & 32.1 & GPS \\
\hline 16150 & 33731 & 21618 & 35 & 55 & 47.3 & -84 & 18 & 30.9 & GPS \\
\hline 16155 & 33753 & 21625 & 35 & 55 & 47.4 & -84 & 18 & 30.7 & GPS \\
\hline 16160 & 33471 & 22582 & 35 & 55 & 53.7 & -84 & 18 & 40.1 & GPS \\
\hline 16165 & 33445 & 22836 & 35 & 55 & 55.7 & -84 & 18 & 42.0 & GPS \\
\hline 16700 & 33732 & 22998 & 35 & 55 & 58.6 & -84 & 18 & 40.2 & GPS \\
\hline 16175 & 34287 & 23111 & 35 & 56 & 2.6 & -84 & 18 & 35.4 & GPS \\
\hline 16180 & 34298 & 23125 & 35 & 56 & 2.8 & -84 & 18 & 35.4 & GPS \\
\hline 16185 & 34392 & 23185 & 35 & 56 & 3.8 & -84 & 18 & 34.8 & GPS \\
\hline 16300 & 34270 & 21611 & 35 & 55 & 50.2 & -84 & 18 & 25.4 & GPS \\
\hline 16305 & 34512 & 21605 & 35 & 55 & 51.4 & -84 & 18 & 22.9 & GPS \\
\hline 16310 & 34511 & 21179 & 35 & 55 & 47.9 & -84 & 18 & 20.1 & GPS \\
\hline 16315 & 34516 & 21159 & 35 & 55 & 47.8 & -84 & 18 & 19.9 & GPS \\
\hline 16320 & 34780 & 20688 & 35 & 55 & 45.4 & -84 & 18 & 14.0 & GPS \\
\hline 16325 & 34797 & 20662 & 35 & 55 & 45.3 & -84 & 18 & 13.7 & GPS \\
\hline 16330 & 34810 & 20622 & 35 & 55 & 45.0 & -84 & 18 & 13.3 & GPS \\
\hline 16335 & 34868 & 20473 & 35 & 55 & 44.1 & -84 & 18 & 11.7 & GPS \\
\hline 16340 & 35283 & 20157 & 35 & 55 & 43.8 & -84 & 18 & 5.3 & GPS \\
\hline 16345 & 35590 & 20046 & 35 & 55 & 44.6 & -84 & 18 & 1.5 & GPS \\
\hline 17005 & 40856 & 19128 & 35 & 56 & 6.0 & -84 & 17 & 2.1 & GPS \\
\hline 17007 & 40851 & 19142 & 35 & 56 & 6.1 & -84 & 17 & 2.2 & DIG \\
\hline 17010 & 40854 & 19368 & 35 & 56 & 7.9 & -84 & 17 & 3.7 & GPS \\
\hline 17015 & 40718 & 19496 & 35 & 56 & 8.2 & -84 & 17 & 5.9 & GPS \\
\hline 17020 & 40748 & 19534 & 35 & 56 & 8.7 & -84 & 17 & 5.9 & GPS \\
\hline 17025 & 40748 & 19667 & 35 & 56 & 9.8 & -84 & 17 & 6.8 & GPS \\
\hline 17030 & 40650 & 19628 & 35 & 56 & 8.9 & -84 & 17 & 7.5 & GPS \\
\hline 17035 & 40559 & 19900 & 35 & 56 & 10.7 & -84 & 17 & 10.3 & GPS \\
\hline 17038 & 40567 & 20231 & 35 & 56 & 13.4 & -84 & 17 & 12.4 & GPS \\
\hline 17040 & 41297 & 20339 & 35 & 56 & 18.3 & -84 & 17 & 5.8 & GPS \\
\hline 17050 & 41558 & 20454 & 35 & 56 & 20.7 & -84 & 17 & 3.9 & GPS \\
\hline 17055 & 41741 & 20116 & 35 & 56 & 18.9 & -84 & 16 & 59.8 & GPS \\
\hline 17060 & 40536 & 20305 & 35 & 56 & 13.9 & -84 & 17 & 13.3 & GPS \\
\hline 17065 & 40549 & 20404 & 35 & 56 & 14.8 & -84 & 17 & 13.8 & GPS \\
\hline 17070 & 40744 & 20961 & 35 & 56 & 20.4 & -84 & 17 & 15.6 & GPS \\
\hline 17075 & 40390 & 20685 & 35 & 56 & 16.2 & -84 & 17 & 17.3 & GPS \\
\hline 17080 & 40534 & 21077 & 35 & 56 & 20.2 & -84 & 17 & 18.5 & GPS \\
\hline 17085 & 40846 & 21332 & 35 & 56 & 24.0 & -84 & 17 & 17.1 & GPS \\
\hline
\end{tabular}


Table 2. Coordinates for seeps, springs, and stream-measurement sites at Oak Ridge National Laboratory--Continued

\begin{tabular}{|c|c|c|c|c|c|c|c|c|c|}
\hline \multirow{3}{*}{$\begin{array}{c}\begin{array}{c}\text { Shte } \\
\text { number }\end{array} \\
17090\end{array}$} & \multicolumn{2}{|c|}{ ORNL coordinates } & \multicolumn{6}{|c|}{ State piane coordinates } & \multirow{3}{*}{$\begin{array}{c}\text { Method } \\
\text { GPS }\end{array}$} \\
\hline & \multirow{2}{*}{$\begin{array}{l}\text { East } \\
40890\end{array}$} & \multirow{2}{*}{$\frac{\text { North }}{21368}$} & \multicolumn{3}{|c|}{ Latitude } & \multicolumn{3}{|c|}{ Longltude } & \\
\hline & & & 35 & 56 & 24.6 & -84 & 17 & 16.9 & \\
\hline 17095 & 41142 & 21567 & 35 & 56 & 27.6 & -84 & 17 & 15.7 & GPS \\
\hline 17100 & 41192 & 21670 & 35 & 56 & 28.7 & -84 & 17 & 15.9 & GPS \\
\hline 17105 & 41228 & 22099 & 35 & 56 & 32.4 & -84 & 17 & 18.4 & GPS \\
\hline 17110 & 41503 & 22351 & 35 & 56 & 36.0 & -84 & 17 & 17.3 & GPS \\
\hline 17115 & 41691 & 22563 & 35 & 56 & 38.8 & -84 & 17 & 16.8 & GPS \\
\hline 17120 & 41880 & 22550 & 35 & 56 & 39.7 & -84 & 17 & 14.8 & GPS \\
\hline 17125 & 42217 & 22836 & 35 & 56 & 43.9 & -84 & 17 & 13.4 & GPS \\
\hline 17130 & 42454 & 23100 & 35 & 56 & 47.4 & -84 & 17 & 12.8 & GPS \\
\hline 17135 & 42649 & 23533 & 35 & 56 & 52.0 & -84 & 17 & 13.7 & GPS \\
\hline 17140 & 42790 & 23775 & 35 & 56 & 54.8 & -84 & 17 & 13.9 & GPS \\
\hline 17145 & 42906 & 24164 & 35 & 56 & 58.6 & -84 & 17 & 15.4 & GPS \\
\hline 18012 & 32507 & 16262 & 35 & 54 & 56.5 & -84 & 18 & 7.1 & DIG \\
\hline 18045 & 32597 & 16275 & 35 & 54 & 57.1 & -84 & 18 & 6.2 & DIG \\
\hline 18050 & 33142 & 16177 & 35 & 54 & 59.3 & -84 & 18 & 0.1 & DIG \\
\hline 18055 & 33299 & 16004 & 35 & 54 & 58.7 & -84 & 17 & 57.3 & DIG \\
\hline 18060 & 33365 & 15993 & 35 & 54 & 59.0 & -84 & 17 & 56.6 & DIG \\
\hline 18065 & 33566 & 16037 & 35 & 55 & 0.5 & -84 & 17 & 54.8 & DIG \\
\hline 18070 & 33668 & 16120 & 35 & 55 & 1.7 & -84 & 17 & 54.4 & DIG \\
\hline 18075 & 33898 & 16300 & 35 & 55 & 4.5 & -84 & 17 & 53.3 & DIG \\
\hline 18080 & 34012 & 16352 & 35 & 55 & 5.5 & -84 & 17 & 52.5 & DIG \\
\hline 18085 & 34094 & 16543 & 35 & 55 & 7.5 & -84 & 17 & 52.9 & DIG \\
\hline 18090 & 34377 & 16994 & 35 & 55 & 12.8 & -84 & 17 & 53.1 & DIG \\
\hline 18095 & 34662 & 17276 & 35 & 55 & 16.7 & -84 & 17 & 52.1 & DIG \\
\hline 18100 & 34789 & 17078 & 35 & 55 & 15.8 & -84 & 17 & 49.5 & DIG \\
\hline 18105 & 34648 & 17372 & 35 & 55 & 17.4 & -84 & 17 & 52.9 & DIG \\
\hline 18110 & 34736 & 17735 & 35 & 55 & 20.9 & -84 & 17 & 54.5 & DIG \\
\hline 18115 & 34718 & 17333 & 35 & 55 & 17.5 & -84 & 17 & 52.0 & DIG \\
\hline 18120 & 35033 & 17403 & 35 & 55 & 19.8 & -84 & 17 & 49.2 & DIG \\
\hline 18125 & 35260 & 17320 & 35 & 55 & 20.3 & -84 & 17 & 46.4 & DIG \\
\hline 18130 & 35312 & 17116 & 35 & 55 & 18.9 & -84 & 17 & 44.5 & DIG \\
\hline 18135 & 35279 & 17459 & 35 & 55 & 21.6 & -84 & 17 & 47.1 & DIG \\
\hline 18140 & 33671 & 15900 & 35 & 54 & 59.9 & -84 & 17 & 52.9 & DIG \\
\hline 18145 & 33987 & 15745 & 35 & 55 & 0.4 & -84 & 17 & 48.6 & DIG \\
\hline 18150 & 34219 & 15824 & 35 & 55 & 2.3 & -84 & 17 & 46.8 & DIG \\
\hline 18155 & 34621 & 15860 & 35 & 55 & 4.8 & -84 & 17 & 43.0 & DIG \\
\hline 18160 & 34687 & 15903 & 35 & 55 & 5.5 & -84 & 17 & 42.6 & DIG \\
\hline 18165 & 34848 & 15865 & 35 & 55 & 6.1 & -84 & 17 & 40.7 & GPS \\
\hline 18170 & 35100 & 15858 & 35 & 55 & 7.4 & -84 & 17 & 38.1 & GPS \\
\hline 18175 & 35514 & 15783 & 35 & 55 & 9.1 & -84 & 17 & 33.4 & DIG \\
\hline 18195 & 36001 & 15457 & 35 & 55 & 9.1 & -84 & 17 & 26.3 & GPS \\
\hline 18200 & 36212 & 15365 & 35 & 55 & 9.5 & -84 & 17 & 23.6 & GPS \\
\hline 18205 & 36281 & 15307 & 35 & 55 & 9.4 & -84 & 17 & 22.5 & GPS \\
\hline 18210 & 36154 & 15263 & 35 & 55 & 8.3 & -84 & 17 & 23.5 & GPS \\
\hline 18215 & 35163 & 15762 & 35 & 55 & 7.0 & -84 & 17 & 36.8 & GPS \\
\hline 18220 & 34722 & 15905 & 35 & 55 & 5.7 & -84 & 17 & 42.3 & GPS \\
\hline 18225 & 34798 & 16054 & 35 & 55 & 7.4 & -84 & 17 & 42.5 & GPS \\
\hline 18230 & 35136 & 15992 & 35 & 55 & 8.7 & -84 & 17 & 38.7 & GPS \\
\hline 18235 & 35492 & 16001 & 35 & 55 & 10.8 & -84 & 17 & 35.1 & GPS \\
\hline 18240 & 35141 & 16080 & 35 & 55 & 9.5 & -84 & 17 & 39.2 & GPS \\
\hline 18245 & 35519 & 16311 & 35 & 55 & 13.5 & -84 & 17 & 37.0 & GPS \\
\hline 18250 & 35490 & 16237 & 35 & 55 & 12.7 & -84 & 17 & 36.8 & DIG \\
\hline 18255 & 35936 & 16063 & 35 & 55 & 13.7 & -84 & 17 & 31.1 & GPS \\
\hline
\end{tabular}


Table 2. Coordinates for seeps, springs, and stream-measurement sites at Oak Ridge National Laboratory--Continued

\begin{tabular}{|c|c|c|c|c|c|c|c|c|c|}
\hline \multirow{3}{*}{$\begin{array}{c}\begin{array}{c}\text { She } \\
\text { number }\end{array} \\
18260\end{array}$} & \multicolumn{2}{|c|}{ ORNL coordinates } & \multicolumn{6}{|c|}{ State plane coordinates } & \multirow{3}{*}{$\frac{\text { Method }}{\text { DIG }}$} \\
\hline & \multirow{2}{*}{$\frac{\text { East }}{36121}$} & \multirow{2}{*}{$\frac{\text { North }}{15918}$} & \multicolumn{3}{|c|}{ Lathude } & \multicolumn{3}{|c|}{ Longltude } & \\
\hline & & & 35 & 55 & 13.5 & -84 & 17 & 28.2 & \\
\hline 18265 & 36372 & 15965 & 35 & 55 & 15.3 & -84 & 17 & 26.0 & GPS \\
\hline 18270 & 36206 & 16131 & 35 & 55 & 15.8 & -84 & 17 & 28.8 & GPS \\
\hline 18275 & 35635 & 16451 & 35 & 55 & 15.3 & -84 & 17 & 36.7 & GPS \\
\hline 18276 & 35620 & 16455 & 35 & 55 & 15.2 & -84 & 17 & 36.9 & DIG \\
\hline 18277 & 35609 & 16587 & 35 & 55 & 16.2 & -84 & 17 & 37.9 & DIG \\
\hline 18280 & 35924 & 16638 & 35 & 55 & 18.4 & -84 & 17 & 35.1 & GPS \\
\hline 18285 & 36239 & 16911 & 35 & 55 & 22.4 & -84 & 17 & 33.7 & GPS \\
\hline 18290 & 36270 & 16870 & 35 & 55 & 22.2 & -84 & 17 & 33.1 & DIG \\
\hline 18295 & 36387 & 16679 & 35 & 55 & 21.3 & -84 & 17 & 30.7 & DIG \\
\hline 18300 & 36639 & 17156 & 35 & 55 & 26.6 & -84 & 17 & 31.4 & DIG \\
\hline 18310 & 37016 & 16770 & 35 & 55 & 25.5 & -84 & 17 & 24.9 & DIG \\
\hline 18315 & 36990 & 17210 & 35 & 55 & 29.0 & -84 & 17 & 28.2 & DIG \\
\hline 18320 & 37423 & 17374 & 35 & 55 & 32.7 & -84 & 17 & 24.9 & DIG \\
\hline 18325 & 37435 & 17287 & 35 & 55 & 32.0 & -84 & 17 & 24.2 & DIG \\
\hline 18330 & 37512 & 16834 & 35 & 55 & 28.7 & -84 & 17 & 20.4 & DIG \\
\hline 18335 & 35730 & 16809 & 35 & 55 & 18.7 & -84 & 17 & 38.2 & DIG \\
\hline 18340 & 35810 & 17277 & 35 & 55 & 23.0 & -84 & 17 & 40.5 & DIG \\
\hline 18345 & 35846 & 17453 & 35 & 55 & 24.7 & -84 & 17 & 41.4 & DIG \\
\hline 18348 & 36050 & 17592 & 35 & 55 & 26.9 & -84 & 17 & 40.2 & DIG \\
\hline 18350 & 36220 & 17609 & 35 & 55 & 28.0 & -84 & 17 & 38.7 & DIG \\
\hline 18355 & 36495 & 17917 & 35 & 55 & 32.0 & -84 & 17 & 38.0 & DIG \\
\hline 18370 & 36223 & 17836 & 35 & 55 & 29.9 & -84 & 17 & 40.2 & DIG \\
\hline 18375 & 35792 & 17490 & 35 & 55 & 24.7 & -84 & 17 & 42.2 & DIG \\
\hline 18380 & 35861 & 17959 & 35 & 55 & 28.9 & -84 & 17 & 44.6 & DIG \\
\hline 18385 & 35861 & 18076 & 35 & 55 & 29.9 & -84 & 17 & 45.4 & DIG \\
\hline 18389 & 36043 & 18163 & 35 & 55 & 31.6 & -84 & 17 & 44.2 & DIG \\
\hline 18390 & 36179 & 18547 & 35 & 55 & 35.5 & -84 & 17 & 45.4 & DIG \\
\hline 18405 & 36086 & 18122 & 35 & 55 & 31.5 & -84 & 17 & 43.5 & DIG \\
\hline 18410 & 36353 & 18550 & 35 & 55 & 36.5 & -84 & 17 & 43.7 & DIG \\
\hline 18415 & 36261 & 19024 & 35 & 55 & 39.8 & -84 & 17 & 47.8 & DIG \\
\hline 18420 & 36335 & 19157 & 35 & 55 & 41.3 & -84 & 17 & 47.9 & DIG \\
\hline 18435 & 36347 & 19401 & 35 & 55 & 43.4 & -84 & 17 & 49.5 & DIG \\
\hline 19001 & 33681 & 16109 & 35 & 55 & 1.7 & -84 & 17 & 54.2 & DIG \\
\hline 19002 & 33754 & 16162 & 35 & 55 & 2.5 & -84 & 17 & 53.8 & DIG \\
\hline 19003 & 33788 & 16181 & 35 & 55 & 2.9 & -84 & 17 & 53.6 & DIG \\
\hline 19004 & 33817 & 16203 & 35 & 55 & 3.2 & -84 & 17 & 53.4 & DIG \\
\hline 19005 & 33853 & 16239 & 35 & 55 & 3.7 & -84 & 17 & 53.3 & DIG \\
\hline 19006 & 33896 & 16290 & 35 & 55 & 4.4 & -84 & 17 & 53.2 & DIG \\
\hline 19007 & 33911 & 16312 & 35 & 55 & 4.6 & -84 & 17 & 53.2 & DIG \\
\hline 19008 & 33914 & 16310 & 35 & 55 & 4.6 & -84 & 17 & 53.2 & DIG \\
\hline 19009 & 34012 & 16356 & 35 & 55 & 5.5 & -84 & 17 & 52.5 & DIG \\
\hline 19010 & 34081 & 16546 & 35 & 55 & 7.5 & -84 & 17 & 53.1 & DIG \\
\hline 19011 & 34139 & 16641 & 35 & 55 & 8.6 & -84 & 17 & 53.1 & DIG \\
\hline 19012 & 34209 & 16735 & 35 & 55 & 9.7 & -84 & 17 & 53.1 & DIG \\
\hline 19013 & 34267 & 16819 & 35 & 55 & 10.8 & -84 & 17 & 53.0 & DIG \\
\hline 19014 & 34274 & 16827 & 35 & 55 & 10.9 & -84 & 17 & 53.0 & DIG \\
\hline 19015 & 34325 & 16895 & 35 & 55 & 11.7 & -84 & 17 & 53.0 & DIG \\
\hline 19016 & 34393 & 17006 & 35 & 55 & 13.0 & -84 & 17 & 53.0 & DIG \\
\hline 19017 & 34431 & 17107 & 35 & 55 & 14.0 & -84 & 17 & 53.3 & DIG \\
\hline 19018 & 34498 & 17199 & 35 & 55 & 15.2 & -84 & 17 & 53.3 & DIG \\
\hline 19019 & 34528 & 17242 & 35 & 55 & 15.7 & -84 & 17 & 53.3 & DIG \\
\hline 19020 & 34589 & 17278 & 35 & 55 & 16.3 & -84 & 17 & 52.9 & DIG \\
\hline
\end{tabular}


Table 2. Coordinates for seeps, springs, and stream-measurement sites at Oak Ridge National Laboratory--Continued

\begin{tabular}{|c|c|c|c|c|c|c|c|c|c|}
\hline \multirow{3}{*}{$\begin{array}{c}\begin{array}{c}\text { Site } \\
\text { number }\end{array} \\
19021\end{array}$} & \multicolumn{2}{|c|}{ ORNL coordinates } & \multicolumn{6}{|c|}{ State plane coordinates } & \multirow{3}{*}{$\begin{array}{c}\text { Method } \\
\text { DIG }\end{array}$} \\
\hline & \multirow{2}{*}{$\begin{array}{l}\text { East } \\
34606\end{array}$} & \multirow{2}{*}{$\frac{\text { North }}{17292}$} & \multicolumn{3}{|c|}{ Latitude } & \multicolumn{3}{|c|}{ Longitude } & \\
\hline & & & 35 & 55 & 16.5 & -84 & 17 & 52.8 & \\
\hline 19022 & 34657 & 17309 & 35 & 55 & 16.9 & -84 & 17 & 52.4 & DIG \\
\hline 19023 & 34661 & 17274 & 35 & 55 & 16.7 & -84 & 17 & 52.1 & DIG \\
\hline 19024 & 34662 & 17270 & 35 & 55 & 16.6 & -84 & 17 & 52.1 & DIG \\
\hline 19025 & 34694 & 17230 & 35 & 55 & 16.5 & -84 & 17 & 51.5 & DIG \\
\hline 19026 & 34725 & 17187 & 35 & 55 & 16.3 & -84 & 17 & 50.9 & DIG \\
\hline 19027 & 34753 & 17140 & 35 & 55 & 16.1 & -84 & 17 & 50.3 & DIG \\
\hline 19028 & 34757 & 17130 & 35 & 55 & 16.0 & -84 & 17 & 50.2 & DIG \\
\hline 19029 & 34783 & 17090 & 35 & 55 & 15.8 & -84 & 17 & 49.7 & DIG \\
\hline 19030 & 34792 & 17073 & 35 & 55 & 15.7 & -84 & 17 & 49.5 & DIG \\
\hline 19031 & 34652 & 17373 & 35 & 55 & 17.4 & -84 & 17 & 52.9 & DIG \\
\hline 19032 & 34644 & 17414 & 35 & 55 & 17.7 & -84 & 17 & 53.2 & DIG \\
\hline 19033 & 34659 & 17496 & 35 & 55 & 18.5 & -84 & 17 & 53.7 & DIG \\
\hline 19034 & 34663 & 17543 & 35 & 55 & 18.9 & -84 & 17 & 53.9 & DIG \\
\hline 19035 & 34662 & 17587 & 35 & 55 & 19.2 & -84 & 17 & 54.2 & DIG \\
\hline 19036 & 34720 & 17707 & 35 & 55 & 20.5 & -84 & 17 & 54.5 & DIG \\
\hline 19037 & 34723 & 17327 & 35 & 55 & 17.4 & -84 & 17 & 51.9 & DIG \\
\hline 19038 & 34777 & 17333 & 35 & 55 & 17.8 & -84 & 17 & 51.4 & DIG \\
\hline 19039 & 34834 & 17339 & 35 & 55 & 18.2 & -84 & 17 & 50.8 & DIG \\
\hline 19040 & 34956 & 17372 & 35 & 55 & 19.1 & -84 & 17 & 49.8 & DIG \\
\hline 19041 & 35035 & 17403 & 35 & 55 & 19.8 & -84 & 17 & 49.2 & DIG \\
\hline 19042 & 35041 & 17448 & 35 & 55 & 20.2 & -84 & 17 & 49.5 & DIG \\
\hline 19043 & 35058 & 17497 & 35 & 55 & 20.7 & -84 & 17 & 49.6 & DIG \\
\hline 19044 & 35072 & 17548 & 35 & 55 & 21.2 & -84 & 17 & 49.8 & DIG \\
\hline 19045 & 35076 & 17595 & 35 & 55 & 21.6 & -84 & 17 & 50.1 & DIG \\
\hline 19046 & 35075 & 17609 & 35 & 55 & 21.7 & -84 & 17 & 50.2 & DIG \\
\hline 19047 & 35254 & 17314 & 35 & 55 & 20.3 & $\cdot-84$ & 17 & 46.4 & DIG \\
\hline 19048 & 35275 & 17267 & 35 & 55 & 20.0 & -84 & 17 & 45.9 & DIG \\
\hline 19049 & 35317 & 17118 & 35 & 55 & 19.0 & -84 & 17 & 44.4 & DIG \\
\hline 19050 & 35279 & 17457 & 35 & 55 & 21.6 & -84 & 17 & 47.1 & DIG \\
\hline 19051 & 35315 & 17503 & 35 & 55 & 22.1 & -84 & 17 & 47.1 & DIG \\
\hline 20001 & 40118 & 21727 & 35 & 56 & 23.3 & -84 & 17 & 27.1 & DIG \\
\hline 20002 & 40111 & 21751 & 35 & 56 & 23.4 & -84 & 17 & 27.3 & DIG \\
\hline 20003 & 40111 & 21751 & 35 & 56 & 23.4 & -84 & 17 & 27.3 & DIG \\
\hline 20004 & 40109 & 21763 & 35 & 56 & 23.5 & -84 & 17 & 27.4 & DIG \\
\hline 20005 & 40104 & 21793 & 35 & 56 & 23.7 & -84 & 17 & 27.7 & DIG \\
\hline 20006 & 40104 & 21821 & 35 & 56 & 24.0 & -84 & 17 & 27.9 & DIG \\
\hline 20007 & 40109 & 21849 & 35 & 56 & 24.2 & -84 & 17 & 28.0 & DIG \\
\hline 20008 & 40115 & 21877 & 35 & 56 & 24.5 & -84 & 17 & 28.1 & DIG \\
\hline 20009 & 40114 & 21903 & 35 & 56 & 24.7 & -84 & 17 & 28.3 & DIG \\
\hline 20010 & 40117 & 21724 & 35 & 56 & 23.2 & -84 & 17 & 27.1 & DIG \\
\hline 20011 & 40068 & 21768 & 35 & 56 & 23.3 & -84 & 17 & 27.9 & DIG \\
\hline 20012 & 40038 & 21793 & 35 & 56 & 23.4 & -84 & 17 & 28.3 & DIG \\
\hline 20013 & 40005 & 21822 & 35 & 56 & 23.4 & -84 & 17 & 28.9 & DIG \\
\hline 20014 & 39992 & 21837 & 35 & 56 & 23.5 & -84 & 17 & 29.1 & DIG \\
\hline 20015 & 39968 & 21860 & 35 & 56 & 23.5 & -84 & 17 & 29.5 & DIG \\
\hline 20016 & 39945 & 21880 & 35 & 56 & 23.6 & -84 & 17 & 29.9 & DIG \\
\hline 20017 & 39905 & 21905 & 35 & 56 & 23.6 & -84 & 17 & 30.5 & DIG \\
\hline 20018 & 39827 & 21957 & 35 & 56 & 23.6 & -84 & 17 & 31.6 & DIG \\
\hline 20019 & 39742 & 22017 & 35 & 56 & 23.6 & -84 & 17 & 32.9 & DIG \\
\hline
\end{tabular}


Table 3. Discharge and water-quality data for the high base flow seepage investigation at Oak Ridge National Laboratory, April 29 through May 10, 1993

[Type of site: Q, stream-measurement sites; SE, seep; SP, spring; /R, site associated with a seepage reach; /A, site is associated with a seepage or spring area; Methods for measurement: $B$, backwater conditions; $D$, no flowing water; $E$, estimation; $L$, less than minimum reportable flow; $P$, pygmy meter measurement; $R$, rating from stream gage; $V$, volumetric measurement; $\mathrm{ft}^{3} / \mathrm{s}$, cubic feet per second; $\mu \mathrm{S} / \mathrm{cm}$, microsiemens per centimeter at $25^{\circ} \mathrm{C}$; ${ }^{\circ} \mathrm{C}$, degree Celsius; --, no data]

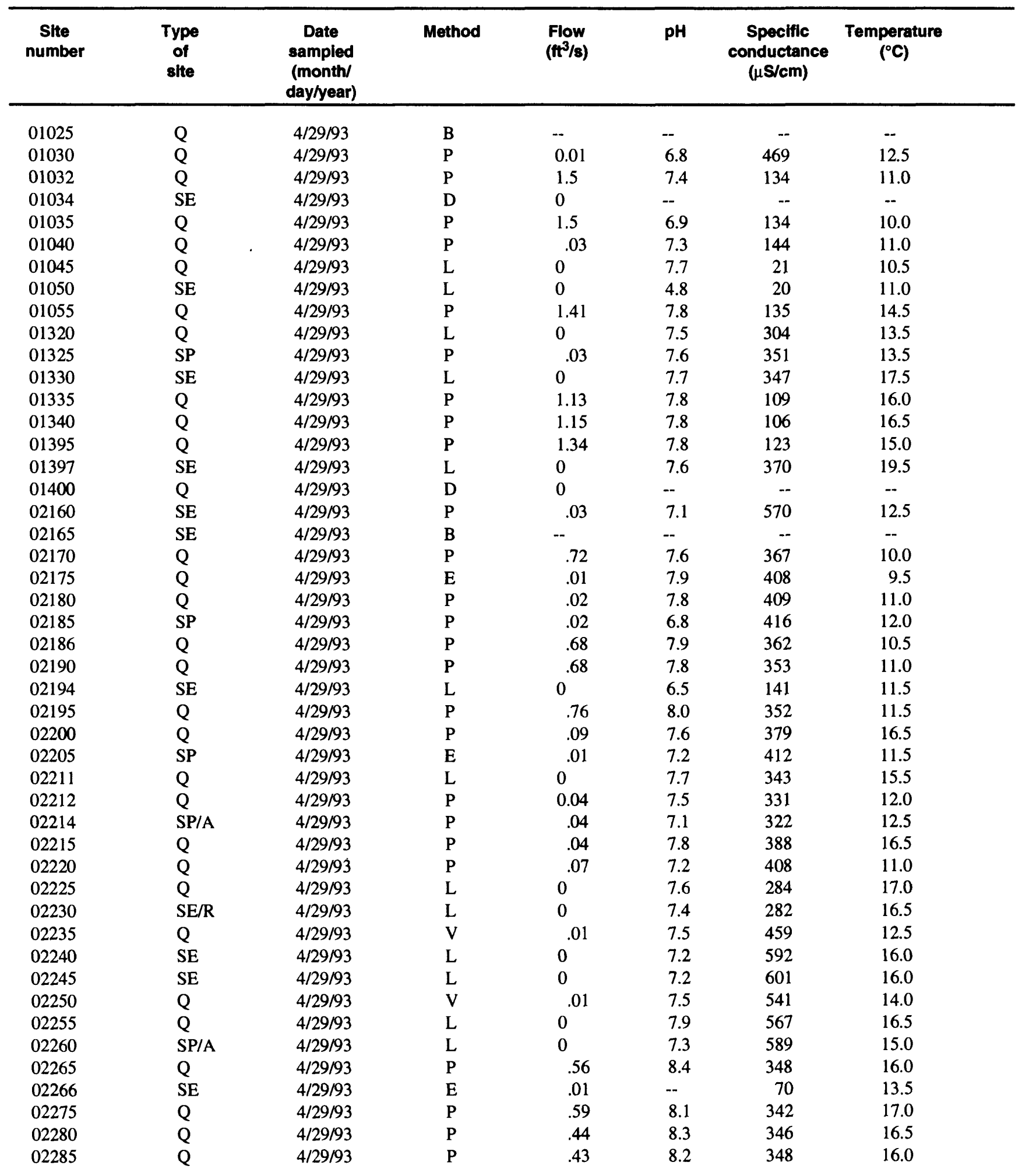


Table 3. Discharge and water-quality data for the high base flow seepage investigation at Oak Ridge National Laboratory, April 29 through May 10, 1993--Continued

\begin{tabular}{|c|c|c|c|c|c|c|c|}
\hline 02290 & $\mathrm{Q}$ & $4 / 29 / 93$ & $\mathbf{P}$ & .18 & 7.9 & 366 & 15.5 \\
\hline 02295 & $Q$ & $4 / 29 / 93$ & $\mathbf{P}$ & .18 & 7.8 & 366 & 15.0 \\
\hline 02310 & SP & $4 / 29 / 93$ & $\mathbf{P}$ & .13 & 7.1 & 363 & 12.0 \\
\hline 02315 & $Q$ & $4 / 29 / 93$ & $P$ & .07 & 7.5 & 366 & 10.5 \\
\hline 02320 & Q & $4 / 29 / 93$ & $\mathbf{P}$ & .05 & 7.3 & 369 & 12.5 \\
\hline 02325 & $\mathrm{Q}$ & $4 / 29 / 93$ & $P$ & .02 & 7.3 & 166 & 13.5 \\
\hline 02330 & SP & $4 / 29 / 93$ & $\mathrm{P}$ & .04 & 7.1 & 330 & 14.0 \\
\hline 02335 & SP & $4 / 29 / 93$ & $\mathrm{P}$ & .02 & 7.2 & 359 & 12.5 \\
\hline 02515 & $\mathrm{Q}$ & $4 / 29 / 93$ & $P$ & .16 & 8.3 & 344 & 17.5 \\
\hline 02520 & $\mathrm{Q}$ & $4 / 29 / 93$ & $\mathbf{P}$ & .16 & 8.2 & 345 & 16.5 \\
\hline 02525 & SP & $4 / 29 / 93$ & $P$ & .06 & 7.1 & 156 & 12.0 \\
\hline 02527 & Q & $4 / 29 / 93$ & $\mathbf{P}$ & .04 & 7.6 & 218 & 12.0 \\
\hline 02528 & SP & $4 / 29 / 93$ & -- & -- & -- & -- & -- \\
\hline 02530 & SE & $4 / 29 / 93$ & $\mathrm{~L}$ & 0 & 6.9 & 309 & 12.0 \\
\hline 02535 & $Q$ & $4 / 29 / 93$ & $\mathbf{L}$ & 0 & 7.0 & 259 & 11.0 \\
\hline 02540 & $\mathrm{Q}$ & $4 / 29 / 93$ & $\mathbf{P}$ & .06 & 7.6 & 225 & 11.5 \\
\hline 02545 & Q & $4 / 29 / 93$ & $\mathbf{P}$ & 0.06 & 7.6 & 230 & 11.0 \\
\hline 02550 & $Q$ & $4 / 29 / 93$ & V & .12 & 7.5 & 389 & 10.5 \\
\hline 02555 & $\mathbf{Q}$ & $4 / 29 / 93$ & $\mathrm{~L}$ & 0 & 7.4 & 430 & 10.0 \\
\hline 02585 & Q & $4 / 29 / 93$ & $\mathbf{P}$ & .04 & 7.9 & 309 & 12.0 \\
\hline 02590 & $\mathbf{Q}$ & $4 / 29 / 93$ & $\mathbf{P}$ & .50 & 7.2 & 387 & 12.0 \\
\hline 02595 & Q & $4 / 29 / 93$ & $\mathbf{F}$ & .03 & 7.3 & 415 & 11.0 \\
\hline 02600 & Q & $4 / 29 / 93$ & D & 0 & -- & -- & -- \\
\hline 02605 & Q & $4 / 29 / 93$ & D & 0 & -- & -- & - \\
\hline 02610 & Q & $4 / 29 / 93$ & D & 0 & -- & -- & -- \\
\hline 02615 & $\mathrm{Q}$ & $4 / 29 / 93$ & $\mathbf{P}$ & .02 & 7.5 & 424 & 14.0 \\
\hline 02620 & SE & $4 / 29 / 93$ & $\mathbf{F}$ & .02 & 7.3 & 428 & 14.0 \\
\hline 02625 & $\mathrm{Q}$ & $4 / 29 / 93$ & $\mathrm{~L}$ & 0 & 7.4 & 86 & 13.0 \\
\hline 02630 & SE & $4 / 29 / 93$ & $\mathrm{~L}$ & 0 & 6.8 & 118 & 13.5 \\
\hline 02635 & $\mathrm{Q}$ & $4 / 29 / 93$ & $\mathrm{~L}$ & 0 & 7.6 & 240 & 14.5 \\
\hline 02640 & SE & $4 / 29 / 93$ & L & 0 & 7.4 & 288 & 20.0 \\
\hline 02645 & SE & $4 / 29 / 93$ & $\mathrm{~L}$ & 0 & 7.1 & 305 & 14.0 \\
\hline 02680 & $\mathrm{SE}$ & $4 / 29 / 93$ & $\mathrm{~L}$ & 0 & 7.2 & 139 & 12.0 \\
\hline 03001 & $\mathrm{Q}$ & $5 / 8 / 93$ & $\mathbf{P}$ & .83 & 7.7 & 279 & 16.0 \\
\hline 03003 & Q & $5 / 8 / 93$ & $\mathbf{P}$ & .75 & 7.8 & 281 & 15.0 \\
\hline 03005 & Q & $5 / 8 / 93$ & $\mathbf{P}$ & .66 & 7.8 & 243 & 15.5 \\
\hline 03050 & $\mathrm{Q}$ & $5 / 8 / 93$ & $\mathbf{P}$ & .41 & 7.6 & 249 & 15.0 \\
\hline 03060 & $Q$ & $5 / 8 / 93$ & $\mathbf{P}$ & .48 & 7.8 & 226 & 14.5 \\
\hline
\end{tabular}


Table 3. Discharge and water-quality data for the high base flow seepage investigation at Oak Ridge National Laboratory, April 29 through May 10, 1993--Continued

\begin{tabular}{|c|c|c|c|c|c|c|c|}
\hline $\begin{array}{c}\text { Site } \\
\text { number }\end{array}$ & $\begin{array}{l}\text { Type } \\
\text { of } \\
\text { site }\end{array}$ & $\begin{array}{c}\text { Date } \\
\text { sampled } \\
\text { (month/ } \\
\text { day/year) } \\
\end{array}$ & Method & $\begin{array}{l}\text { Fiow } \\
\left(\mathrm{ft}^{3} / \mathrm{s}\right)\end{array}$ & pH & $\begin{array}{c}\text { Specific } \\
\text { conductance } \\
(\mu \mathrm{S} / \mathrm{cm})\end{array}$ & $\begin{array}{c}\text { Temperature } \\
\left({ }^{\circ} \mathrm{C}\right)\end{array}$ \\
\hline 03065 & SE & $5 / 8 / 93$ & D & 0 & -- & -- & -- \\
\hline 03070 & SP & $5 / 8 / 93$ & D & 0 & -- & -. & -- \\
\hline 03075 & SP & $5 / 8 / 93$ & $\mathbf{P}$ & .07 & 7.1 & 204 & 13.0 \\
\hline 03080 & SE & $5 / 8 / 93$ & D & 0 & -- & -- & -- \\
\hline 03085 & SP & $5 / 8 / 93$ & E & .02 & 7.2 & 244 & 13.0 \\
\hline 03090 & SP & $5 / 8 / 93$ & $\mathbf{P}$ & .02 & 7.4 & 255 & 13.5 \\
\hline 03095 & SE & $5 / 8 / 93$ & D & 0 & -- & -. & -- \\
\hline 03100 & SP & $5 / 8 / 93$ & P & 0.24 & 6.9 & 207 & 13.5 \\
\hline 03110 & $\mathrm{Q}$ & $5 / 8 / 93$ & V & .01 & 7.7 & 318 & 20.5 \\
\hline 03115 & $\mathrm{Q}$ & $5 / 8 / 93$ & V & .02 & 7.9 & 269 & 22.0 \\
\hline 03485 & $\mathrm{SE}$ & $5 / 8 / 93$ & D & 0 & -- & -- & -- \\
\hline 03490 & SE & $5 / 8 / 93$ & D & 0 & -- & -- & -- \\
\hline 03495 & $\mathrm{Q}$ & $5 / 8 / 93$ & D & 0 & -- & -- & -- \\
\hline 03500 & SP & $5 / 8 / 93$ & D & 0 & -- & -- & -- \\
\hline 03510 & $\mathrm{Q}$ & $5 / 8 / 93$ & $\mathbf{P}$ & .32 & 8.2 & 310 & 17.5 \\
\hline 03515 & Q & $5 / 8 / 93$ & $\mathbf{P}$ & .02 & 7.5 & 278 & 19.0 \\
\hline 03520 & $\mathrm{Q}$ & $5 / 8 / 93$ & $\mathbf{P}$ & .21 & 8.1 & 370 & 17.0 \\
\hline 03525 & $\mathrm{Q}$ & $5 / 8 / 93$ & $\mathbf{P}$ & .05 & 7.9 & 250 & 22.0 \\
\hline 03530 & $\mathrm{Q}$ & $5 / 8 / 93$ & $\mathbf{P}$ & .02 & 8.5 & 275 & 22.5 \\
\hline 03535 & $\mathrm{Q}$ & $5 / 8 / 93$ & $\mathbf{P}$ & .13 & 7.8 & 450 & 14.5 \\
\hline 03537 & SE & $5 / 8 / 93$ & D & 0 & -- & -- & - \\
\hline 03538 & SE & $5 / 8 / 93$ & D & 0 & -- & -- & -- \\
\hline 03540 & $\mathrm{Q}$ & $5 / 8 / 93$ & $\mathbf{P}$ & .08 & 7.7 & 243 & 15.5 \\
\hline 03545 & SE & $5 / 8 / 93$ & L & 0 & 7.3 & 415 & 18.0 \\
\hline 03550 & SP & $5 / 8 / 93$ & $\mathbf{P}$ & .03 & 7.5 & 376 & 17.5 \\
\hline 03555 & SP & $5 / 8 / 93$ & D & 0 & -- & -- & -- \\
\hline 03560 & $\mathrm{Q}$ & $5 / 8 / 93$ & V & .01 & 8.3 & 375 & 17.0 \\
\hline 03565 & SP & $5 / 8 / 93$ & $P$ & .04 & 7.5 & 335 & 13.0 \\
\hline 03570 & SP & $5 / 8 / 93$ & D & 0 & -- & -- & -- \\
\hline 03575 & Q & $5 / 8 / 93$ & D & 0 & -- & -- & - \\
\hline 03580 & $\mathrm{SP}$ & $5 / 8 / 93$ & $\mathbf{L}$ & 0 & 7.2 & 45 & 18.5 \\
\hline 03585 & SE & $5 / 8 / 93$ & D & 0 & -- & -- & -- \\
\hline 03590 & Q & $5 / 8 / 93$ & $P$ & .03 & 7.9 & 456 & 19.5 \\
\hline 03591 & $\mathrm{Q}$ & $5 / 8 / 93$ & D & 0 & -- & - & -- \\
\hline 03592 & Q & $5 / 8 / 93$ & D & 0 & -- & -- & -- \\
\hline 03593 & SE & $5 / 8 / 93$ & D & 0 & -- & -- & -- \\
\hline 03594 & SE & $5 / 8 / 93$ & D & 0 & -- & -- & -- \\
\hline 03595 & SE & $5 / 8 / 93$ & D & 0 & -- & -- & -- \\
\hline 03598 & Q & $5 / 8 / 93$ & L & 0 & 7.2 & 360 & 15.5 \\
\hline 03600 & SE & $5 / 8 / 93$ & -- & -- & -. & -- & -- \\
\hline 03605 & SE & $5 / 8 / 93$ & D & 0 & -- & -- & -- \\
\hline 03610 & Q & $5 / 8 / 93$ & $\mathrm{~L}$ & 0 & 8.3 & 177 & 18.0 \\
\hline 03615 & SE & $5 / 8 / 93$ & $\mathrm{~L}$ & 0 & 7.2 & 159 & 13.5 \\
\hline 03620 & SE & $5 / 8 / 93$ & $\mathrm{~L}$ & 0 & 7.8 & 217 & 14.5 \\
\hline 03625 & Q & $5 / 8 / 93$ & $\mathbf{P}$ & 0.02 & 6.9 & 641 & 14.5 \\
\hline 03630 & $\mathrm{Q}$ & $5 / 8 / 93$ & D & 0 & -- & -- & -- \\
\hline 03635 & $\mathrm{Q}$ & $5 / 8 / 93$ & D & 0 & -- & -- & -- \\
\hline 03640 & Q & $5 / 8 / 93$ & D & 0 & -- & -- & -- \\
\hline 03645 & SE & $5 / 8 / 93$ & $\mathbf{L}$ & 0 & 7.6 & 555 & 15.0 \\
\hline 03648 & SE & $5 / 8 / 93$ & D & 0 & -- & -- & -- \\
\hline 03650 & SE & $5 / 8 / 93$ & D & 0 & - & -- & -- \\
\hline 03654 & SE & $5 / 8 / 93$ & D & 0 & -- & -- & -- \\
\hline
\end{tabular}


Table 3. Discharge and water-quality data for the high base flow seepage investigation at Oak Ridge National Laboratory, April 29 through May 10, 1993--Continued

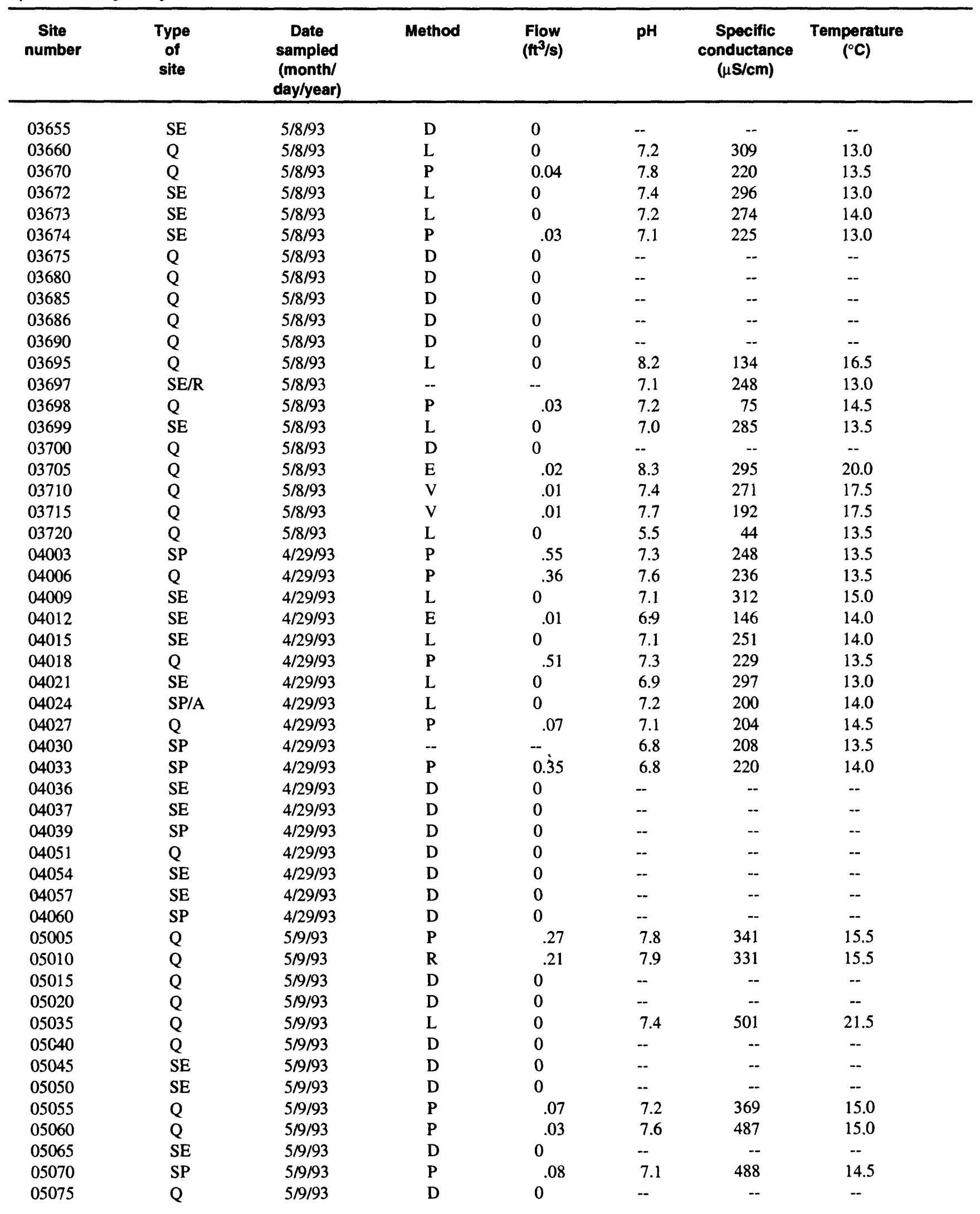


Table 3. Discharge and water-quality data for the high base flow seepage investigation at Oak Ridge National Laboratory, April 29 through May 10, 1993--Continued

\begin{tabular}{|c|c|c|c|c|c|c|c|}
\hline $\begin{array}{c}\text { Site } \\
\text { number }\end{array}$ & $\begin{array}{l}\text { Type } \\
\text { of } \\
\text { site }\end{array}$ & $\begin{array}{c}\text { Date } \\
\text { sampled } \\
\text { (month/ } \\
\text { day/year) }\end{array}$ & Method & $\begin{array}{l}\text { Flow } \\
\left(\mathrm{ft}^{3} / \mathrm{s}\right)\end{array}$ & pH & $\begin{array}{c}\text { Speciflc } \\
\text { conductance } \\
(\mu \mathrm{S} / \mathrm{cm})\end{array}$ & $\begin{array}{c}\text { Temperature } \\
\left({ }^{\circ} \mathrm{C}\right)\end{array}$ \\
\hline 05077 & SE & $5 / 9 / 93$ & $\mathrm{~L}$ & 0 & 7.8 & 518 & 18.5 \\
\hline 05080 & SE & $5 / 9 / 93$ & D & 0 & -- & -- & -- \\
\hline 05085 & $\mathrm{Q}$ & $5 / 9 / 93$ & $\mathbf{P}$ & .03 & 8.2 & 318 & 15.5 \\
\hline 05090 & $\mathrm{Q}$ & $5 / 9 / 93$ & $P$ & .06 & 8.2 & 313 & 15.5 \\
\hline 05095 & $\mathrm{SE}$ & $5 / 9 / 93$ & D & 0 & -- & -- & -- \\
\hline 05100 & $\mathrm{SE}$ & $5 / 9 / 93$ & $\mathrm{D}$ & 0 & -- & .- & -- \\
\hline 05105 & $\mathrm{Q}$ & $5 / 9 / 93$ & $\mathbf{P}$ & .05 & 7.8 & 306 & 16.0 \\
\hline 05108 & SE & $5 / 9 / 93$ & D & 0 & $\ldots$ & -. & - \\
\hline 05110 & $\mathrm{Q}$ & $5 / 9 / 93$ & $P$ & .05 & 7.9 & 261 & 14.5 \\
\hline 05112 & SE & $5 / 9 / 93$ & $\mathrm{~L}$ & 0 & 5.3 & 59 & 16.5 \\
\hline 05115 & SP & $5 / 9 / 93$ & $\mathbf{P}$ & .04 & 7.0 & 255 & 13.0 \\
\hline 05120 & $\mathrm{Q}$ & $5 / 9 / 93$ & $\mathrm{~L}$ & 0 & 7.0 & 107 & 15.0 \\
\hline 05125 & SE & $5 / 9 / 93$ & D & 0 & -- & -- & -- \\
\hline 05130 & $\mathrm{Q}$ & $5 / 9 / 93$ & L & 0 & 7.0 & 54 & 15.5 \\
\hline 05135 & SP & $5 / 9 / 93$ & L & 0 & 6.5 & 49 & 14.5 \\
\hline 05150 & SE & $5 / 9 / 93$ & D & 0 & -- & -- & -- \\
\hline 05155 & $\mathrm{Q}$ & $5 / 9 / 93$ & $\mathrm{P}$ & 0.10 & 7.1 & 267 & 16.0 \\
\hline 05160 & $Q$ & $5 / 9 / 93$ & D & 0 & -. & -- & -- \\
\hline 05165 & SE & $5 / 9 / 93$ & D & 0 & -- & -- & -. \\
\hline 05170 & $\mathrm{Q}$ & $5 / 9 / 93$ & $\mathrm{D}$ & 0 & -- & -- & -- \\
\hline 05175 & SE & $5 / 9 / 93$ & D & 0 & -- & -- & -- \\
\hline 05180 & $\mathrm{Q}$ & $5 / 9 / 93$ & $P$ & .09 & 7.8 & 261 & 15.5 \\
\hline 05182 & $\mathrm{Q}$ & $5 / 9 / 93$ & $\mathrm{~L}$ & 0 & 7.3 & 457 & 15.0 \\
\hline 05183 & $Q$ & $5 / 9 / 93$ & $\mathrm{~L}$ & 0 & 7.8 & 254 & 16.5 \\
\hline 05184 & $Q$ & $5 / 9 / 93$ & D & 0 & .- & -. & -- \\
\hline 05188 & $\mathrm{Q}$ & $5 / 9 / 93$ & D & 0 & -- & -- & -. \\
\hline 05190 & $\mathrm{Q}$ & $5 / 9 / 93$ & $\mathrm{P}$ & .02 & 7.7 & 343 & 17.5 \\
\hline 05192 & $\mathrm{Q}$ & $5 / 9 / 93$ & $P$ & .02 & 7.8 & 241 & 15.0 \\
\hline 05195 & $Q$ & $5 / 9 / 93$ & $\mathrm{P}$ & .07 & 8.0 & 278 & 19.0 \\
\hline 05197 & $\mathrm{Q}$ & $5 / 9 / 93$ & D & 0 & -- & -- & -- \\
\hline 05199 & SE & $5 / 9 / 93$ & D & 0 & -- & -- & -- \\
\hline 05200 & $\mathrm{Q}$ & $5 / 9 / 93$ & $\mathbf{P}$ & .08 & 8.0 & 217 & 15.5 \\
\hline 05209 & $\mathrm{Q}$ & $5 / 9 / 93$ & D & 0 & - & -- & -- \\
\hline 05212 & SE & $5 / 9 / 93$ & D & 0 & -- & -- & -- \\
\hline 05218 & SE & $5 / 9 / 93$ & D & 0 & -. & -- & -. \\
\hline 05219 & $\mathbf{Q}$ & $5 / 9 / 93$ & $P$ & .16 & 8.2 & 217 & 15.0 \\
\hline 05221 & Q & $5 / 9 / 93$ & D & 0 & -- & -- & -- \\
\hline 05224 & $\mathrm{Q}$ & $5 / 9 / 93$ & D & 0 & -. & -- & -- \\
\hline 05227 & SE & $5 / 9 / 93$ & D & 0 & -- & -. & -- \\
\hline 05230 & SE & $5 / 9 / 93$ & $\mathrm{D}$ & 0 & -- & -- & -- \\
\hline 05236 & SE & $5 / 9 / 93$ & D & 0 & -- & -- & -- \\
\hline 05239 & SE & $5 / 9 / 93$ & D & 0 & - & -- & -- \\
\hline 05242 & SE & $5 / 9 / 93$ & L & 0 & 7.5 & 608 & 20.5 \\
\hline 05245 & $\mathrm{Q}$ & $5 / 9 / 93$ & $\mathbf{P}$ & .46 & 7.2 & 217 & 15.0 \\
\hline 05247 & SE & $5 / 9 / 93$ & -- & - & 7.1 & 655 & 14.5 \\
\hline 05248 & $\mathrm{Q}$ & $5 / 9 / 93$ & $\mathbf{P}$ & .45 & 8.1 & 202 & 15.5 \\
\hline 05251 & $\mathrm{Q}$ & $5 / 9 / 93$ & D & 0 & -- & -- & -- \\
\hline 05255 & $\mathrm{Q}$ & $5 / 9 / 93$ & D & 0 & -- & -- & -- \\
\hline 05260 & SE & $5 / 9 / 93$ & $\mathrm{D}$ & 0 & -- & -- & -- \\
\hline 05265 & Q & $5 / 9 / 93$ & $P$ & .48 & 7.8 & 212 & 13.5 \\
\hline 05270 & $\mathrm{Q}$ & $5 / 9 / 93$ & $\mathrm{E}$ & .01 & 7.5 & 368 & 15.5 \\
\hline 05275 & Q & $5 / 9 / 93$ & $\mathrm{D}$ & 0 & -- & -- & -- \\
\hline
\end{tabular}


Table 3. Discharge and water-quality data for the high base flow seepage investigation at Oak Ridge National Laboratory, April 29 through May 10, 1993--Continued

\begin{tabular}{|c|c|c|c|c|c|c|c|}
\hline $\begin{array}{c}\text { Site } \\
\text { number }\end{array}$ & $\begin{array}{l}\text { Type } \\
\text { of } \\
\text { site }\end{array}$ & $\begin{array}{c}\text { Date } \\
\text { sampled } \\
\text { (month/ } \\
\text { day/year) }\end{array}$ & Method & $\begin{array}{l}\text { Flow } \\
\left(\mathrm{ft}^{3} / \mathrm{s}\right)\end{array}$ & pH & $\begin{array}{c}\text { Specific } \\
\text { conductance } \\
(\mu \mathrm{S} / \mathrm{cm})\end{array}$ & $\begin{array}{c}\text { Temperature } \\
\left({ }^{\circ} \mathrm{C}\right)\end{array}$ \\
\hline 05278 & $\mathrm{Q}$ & $5 / 9 / 93$ & D & 0 & -- & -- & -- \\
\hline 05280 & SE & $5 / 9 / 93$ & D & 0 & -- & -- & -- \\
\hline 05285 & $\mathrm{Q}$ & $5 / 9 / 93$ & D & 0 & -- & -- & -- \\
\hline 05290 & SP & $5 / 9 / 93$ & $\mathbf{P}$ & 0.06 & 6.8 & 163 & 12.5 \\
\hline 05295 & $\mathrm{SE} / \mathrm{R}$ & $5 / 9 / 93$ & L & 0 & 7.2 & 244 & 14.0 \\
\hline 05300 & $\mathrm{Q}$ & $5 / 9 / 93$ & $\mathbf{P}$ & .40 & 7.9 & 217 & 14.5 \\
\hline 05305 & $\mathrm{Q}$ & $5 / 9 / 93$ & $\mathbf{P}$ & .23 & 8.1 & 236 & 15.0 \\
\hline 05310 & SE & $5 / 9 / 93$ & $\mathbf{L}$ & 0 & 6.9 & 159 & 15.0 \\
\hline 05315 & SP & $5 / 9 / 93$ & $\mathbf{P}$ & .01 & 7.2 & 202 & 13.5 \\
\hline 05320 & SE & $5 / 9 / 93$ & L & 0 & 6.9 & 146 & 12.5 \\
\hline 05325 & SP & $5 / 9 / 93$ & $\mathbf{P}$ & .02 & 6.9 & 146 & 12.5 \\
\hline 05328 & SE & $5 / 9 / 93$ & D & 0 & -- & -- & -- \\
\hline 05330 & SP & $5 / 9 / 93$ & $\mathbf{P}$ & .08 & 6.9 & 147 & 12.5 \\
\hline 05335 & SP & $5 / 9 / 93$ & $P$ & .04 & 6.9 & 150 & 12.5 \\
\hline 05340 & SE & $5 / 9 / 93$ & D & 0 & -- & -- & -- \\
\hline 05345 & SE & $5 / 9 / 93$ & $\mathrm{~L}$ & 0 & 6.5 & 104 & 16.5 \\
\hline 05350 & SE & $5 / 9 / 93$ & D & 0 & -- & -- & -- \\
\hline 05355 & $Q$ & $5 / 9 / 93$ & $\mathbf{P}$ & .07 & 7.4 & 282 & 13.0 \\
\hline 06005 & $\mathrm{Q}$ & $5 / 1 / 93$ & $P$ & .27 & 7.4 & 311 & 12.0 \\
\hline 06010 & SP & $5 / 1 / 93$ & L & 0 & 6.3 & 140 & 14.0 \\
\hline 06015 & $\mathrm{Q}$ & $5 / 1 / 93$ & $\mathbf{P}$ & .09 & 7.6 & 328 & 12.0 \\
\hline 06020 & $\mathrm{Q}$ & $5 / 1 / 93$ & D & 0 & -- & -- & -- \\
\hline 06025 & $\mathrm{Q}$ & $5 / 1 / 93$ & $\mathrm{~L}$ & 0 & 7.8 & 127 & 12.5 \\
\hline 06030 & $\mathrm{Q}$ & $5 / 1 / 93$ & $\mathbf{P}$ & .02 & 7.6 & 123 & 12.5 \\
\hline 06040 & $\mathrm{Q}$ & $5 / 1 / 93$ & $\mathbf{P}$ & .05 & 7.1 & 121 & 12.5 \\
\hline 06042 & $\mathrm{Q}$ & $5 / 1 / 93$ & $\mathbf{E}$ & .01 & 7.0 & 216 & 12.5 \\
\hline 06044 & SE & $5 / 1 / 93$ & D & 0 & -- & - & -. \\
\hline 06045 & SE & $5 / 1 / 93$ & E & .01 & 6.5 & 79 & 11.5 \\
\hline 06048 & SP & $5 / 1 / 93$ & $\mathbf{P}$ & 0.03 & 6.0 & 48 & 12.0 \\
\hline 06050 & SE & $5 / 1 / 93$ & D & 0 & -- & -- & -- \\
\hline 06055 & $\mathrm{Q}$ & $5 / 1 / 93$ & $\mathbf{P}$ & .14 & 7.8 & 319 & 12.0 \\
\hline 06056 & SE & $5 / 1 / 93$ & L & 0 & 6.8 & 417 & 12.5 \\
\hline 06060 & $\mathrm{Q}$ & $5 / 1 / 93$ & $\mathbf{P}$ & .1 & 7.5 & 312 & 13.0 \\
\hline 06065 & $\mathrm{Q}$ & $5 / 1 / 93$ & D & 0 & -- & -- & -- \\
\hline 06070 & SP & $5 / 1 / 93$ & $\mathbf{P}$ & .03 & 7.3 & 320 & 16.0 \\
\hline 06075 & SP & $5 / 1 / 93$ & $\mathbf{P}$ & .1 & 7.1 & 308 & 13.5 \\
\hline 06080 & $\mathrm{Q}$ & $5 / 1 / 93$ & $\mathbf{P}$ & .01 & 7.7 & 423 & 12.5 \\
\hline 06085 & 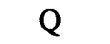 & $5 / 1 / 93$ & $\mathbf{P}$ & .02 & 7.0 & 447 & 12.5 \\
\hline 06087 & $Q$ & $5 / 1 / 93$ & $\mathbf{P}$ & .02 & 7.3 & 191 & 14.0 \\
\hline 06090 & $\mathrm{Q}$ & $5 / 1 / 93$ & D & 0 & - & -- & -- \\
\hline 06095 & $\mathrm{Q}$ & $5 / 1 / 93$ & $\mathbf{P}$ & 0.06 & 7.4 & 237 & 13.5 \\
\hline 06100 & SP & $5 / 1 / 93$ & $\mathbf{P}$ & .18 & 7.1 & 238 & 14.5 \\
\hline 07005 & SE & $5 / 7 / 93$ & D & 0 & -- & -- & -- \\
\hline 07010 & $\mathrm{Q}$ & $5 / 7 / 93$ & D & 0 & -- & - & -- \\
\hline 07015 & SP & $5 / 7 / 93$ & D & 0 & -- & -- & -- \\
\hline 07020 & $\mathrm{Q}$ & $5 / 7 / 93$ & $\mathrm{~L}$ & 0 & 6.0 & 97 & 12.5 \\
\hline 07021 & $\mathrm{SE}$ & $5 / 7 / 93$ & -- & -- & 5.9 & 58 & 12.5 \\
\hline 07022 & $Q$ & $5 / 7 / 93$ & $\mathrm{~L}$ & 0 & 6.9 & 63 & 14.5 \\
\hline 07025 & $\mathrm{Q}$ & $5 / 7 / 93$ & V & .01 & 6.6 & 39 & 15.0 \\
\hline 07030 & SP & $5 / 7 / 93$ & $\mathbf{L}$ & 0 & 5.9 & 28 & 15.0 \\
\hline 07035 & $\mathrm{Q}$ & $5 / 7 / 93$ & L & 0 & -- & 122 & 14.5 \\
\hline 07038 & SE & $5 / 7 / 93$ & V & .01 & 6.1 & 92 & 14.0 \\
\hline
\end{tabular}


Table 3. Discharge and water-quality data for the high base flow seepage investigation at Oak Ridge National Laboratory, April 29 through May 10, 1993--Continued

\begin{tabular}{|c|c|c|c|c|c|c|c|}
\hline $\begin{array}{c}\text { Site } \\
\text { number }\end{array}$ & $\begin{array}{l}\text { Type } \\
\text { of } \\
\text { site }\end{array}$ & $\begin{array}{c}\text { Date } \\
\text { sampled } \\
\text { (month/ } \\
\text { day/year) }\end{array}$ & Method & $\begin{array}{l}\text { Flow } \\
\left(\mathrm{ft}^{3} / \mathrm{s}\right)\end{array}$ & pH & $\begin{array}{c}\text { Specific } \\
\text { conductance } \\
(\mu \mathrm{S} / \mathrm{cm})\end{array}$ & $\begin{array}{l}\text { Temperature } \\
\left({ }^{\circ} \mathrm{C}\right)\end{array}$ \\
\hline 07040 & Q & $5 / 7 / 93$ & D & 0 & -- & -- & -- \\
\hline 07045 & $\mathrm{Q}$ & $5 / 7 / 93$ & D & 0 & -- & -- & .. \\
\hline 07050 & SE & $5 / 7 / 93$ & D & 0 & -- & -. & -- \\
\hline 07100 & Q & $5 / 7 / 93$ & P & .1 & 6.7 & 218 & 16.0 \\
\hline 07105 & Q & $5 / 7 / 93$ & L & 0 & 7.4 & 230 & 14.5 \\
\hline 07110 & SE & $5 / 7 / 93$ & D & 0 & -- & -- & -- \\
\hline 07115 & $\mathrm{Q}$ & $5 / 7 / 93$ & L & 0 & 7.2 & 207 & 14.5 \\
\hline 07120 & SE & $5 / 7 / 93$ & L & 0 & 6.7 & 240 & 14.0 \\
\hline 07125 & $\mathrm{Q}$ & $5 / 7 / 93$ & $\mathbf{P}$ & .07 & 7.9 & 218 & 16.0 \\
\hline 07130 & $\mathrm{Q}$ & $5 / 7 / 93$ & L & 0 & 6.9 & 81 & 14.5 \\
\hline 07135 & Q & $5 / 7 / 93$ & L & 0 & 6.8 & 55 & 15.0 \\
\hline 07140 & $\mathrm{Q}$ & $5 / 7 / 93$ & L & 0 & 7.1 & 79 & 14.0 \\
\hline 07145 & $\mathrm{SE} / \mathrm{R}$ & $5 / 7 / 9.3$ & D & 0 & -- & -- & -- \\
\hline 07200 & $\mathrm{Q}$ & $5 / 7 / 93$ & L & 0 & 6.7 & 100 & 14.0 \\
\hline 07205 & $\mathrm{Q}$ & $5 / 7 / 93$ & L & 0 & 6.2 & 121 & 14.0 \\
\hline 07210 & $\mathrm{Q}$ & $5 / 7 / 93$ & $P$ & .06 & 7.9 & 237 & 14.5 \\
\hline 07215 & $\mathrm{Q}$ & $5 / 7 / 93$ & $\mathrm{~L}$ & 0 & 7.6 & 236 & 14.0 \\
\hline 07220 & SE & $5 / 7 / 93$ & D & 0 & -- & -- & -. \\
\hline 07225 & $\mathrm{Q}$ & $5 / 7 / 93$ & D & 0 & -- & -- & -- \\
\hline 07228 & SE & $5 / 7 / 93$ & $\mathrm{~L}$ & 0 & 7.3 & & 15.0 \\
\hline 07230 & SE & $5 / 7 / 93$ & D & 0 & -- & -- & -- \\
\hline 07235 & $\mathrm{Q}$ & $5 / 7 / 93$ & $P$ & .07 & 8.0 & 243 & 15.0 \\
\hline 07240 & $\mathrm{Q}$ & $5 / 7 / 93$ & $\mathbf{P}$ & .01 & 7.5 & 124 & 14.5 \\
\hline 07245 & $\mathbf{Q}$ & $5 / 7 / 93$ & E & .01 & 7.4 & 106 & 13.5 \\
\hline 07250 & $\mathrm{Q}$ & $5 / 7 / 93$ & E & .01 & 7.1 & 126 & 15.0 \\
\hline 07255 & $\mathrm{Q}$ & $5 / 7 / 93$ & D & 0 & -- & -- & -- \\
\hline 07260 & SE & $5 / 7 / 93$ & D & 0 & -- & -- & -- \\
\hline 07300 & $\mathrm{Q}$ & $5 / 7 / 93$ & L & 0 & 7.3 & 133 & 14.5 \\
\hline 07345 & $\mathrm{Q}$ & $5 / 7 / 93$ & L & 0 & 7.6 & 42 & 17.0 \\
\hline 07350 & SE & $5 / 7 / 93$ & D & 0 & -- & -- & -- \\
\hline 07353 & SE & $5 / 7 / 93$ & D & 0 & -- & -- & -- \\
\hline 07355 & $\mathrm{Q}$ & $5 / 7 / 93$ & $\mathbf{P}$ & 0.03 & 8.0 & 275 & 18.0 \\
\hline 07360 & SE & $5 / 7 / 93$ & D & 0 & -- & -- & - \\
\hline 07362 & $\mathrm{Q}$ & $5 / 7 / 93$ & V & .01 & 7.8 & 392 & 15.0 \\
\hline 07365 & $\mathrm{Q}$ & $5 / 7 / 93$ & V & .01 & 7.2 & 397 & 15.0 \\
\hline 07370 & $\mathrm{Q}$ & $5 / 7 / 93$ & $P$ & .02 & 7.9 & 276 & 18.5 \\
\hline 07375 & Q & $5 / 7 / 93$ & $\mathbf{P}$ & .02 & 8.2 & 269 & 18.0 \\
\hline 07380 & $\mathrm{Q}$ & $5 / 7 / 93$ & $\mathrm{~L}$ & 0 & 6.3 & 163 & 13.0 \\
\hline 07385 & SE & $5 / 7 / 93$ & D & 0 & -- & -- & -- \\
\hline 07395 & SE & $5 / 7 / 93$ & D & 0 & -- & -- & -- \\
\hline 07400 & $\mathrm{Q}$ & $5 / 7 / 93$ & V & .01 & 8.1 & 213 & 16.5 \\
\hline 07405 & SE & $5 / 7 / 93$ & D & 0 & -- & -- & -- \\
\hline 07410 & SP & $5 / 7 / 93$ & L & 0 & 6.6 & 556 & 14.5 \\
\hline 07415 & $Q$ & $5 / 7 / 93$ & $P$ & .02 & 7.6 & 183 & 16.0 \\
\hline 07420 & Q & $5 / 7 / 93$ & $P$ & .02 & 7.2 & 65 & 17.0 \\
\hline 07425 & $\mathrm{Q}$ & $5 / 7 / 93$ & L & 0 & 7.5 & 71 & 16.5 \\
\hline 07430 & SP & $5 / 7 / 93$ & $\mathrm{~L}$ & 0 & 6.5 & 57 & 14.0 \\
\hline 08005 & $\mathrm{Q}$ & $5 / 10 / 93$ & D & 0 & -- & -- & -- \\
\hline 08010 & $\mathrm{SE}$ & $5 / 10 / 93$ & $\mathrm{D}$ & 0 & -- & -- & - \\
\hline 08013 & SE & $5 / 10 / 93$ & D & 0 & -- & -- & -- \\
\hline 08015 & SE & $5 / 10 / 93$ & D & 0 & -- & -- & -- \\
\hline 08020 & SE & $5 / 10 / 93$ & L & 0 & 6.0 & 200 & 16.0 \\
\hline
\end{tabular}


Table 3. Discharge and water-quality data for the high base flow seepage investigation at Oak Ridge National Laboratory, April 29 through May 10, 1993--Continued

\begin{tabular}{|c|c|c|c|c|c|c|c|}
\hline $\begin{array}{c}\text { Site } \\
\text { number }\end{array}$ & $\begin{array}{l}\text { Type } \\
\text { of } \\
\text { site }\end{array}$ & $\begin{array}{c}\text { Date } \\
\text { sampled } \\
\text { (month/ } \\
\text { day/year) }\end{array}$ & Method & $\begin{array}{l}\text { Flow } \\
\left(\mathrm{ft}^{3} / \mathrm{s}\right)\end{array}$ & $\mathrm{pH}$ & $\begin{array}{c}\text { Specific } \\
\text { conductance } \\
(\mu \mathrm{S} / \mathrm{cm})\end{array}$ & $\begin{array}{c}\text { Temperature } \\
\left({ }^{\circ} \mathrm{C}\right)\end{array}$ \\
\hline 08025 & SE & $5 / 10 / 93$ & $\mathbf{L}$ & 0 & 6.8 & 186 & 14.5 \\
\hline 08028 & SE & $5 / 10 / 93$ & $\bar{D}$ & 0 & -. & -. & -- \\
\hline 08030 & $\mathrm{Q}$ & $5 / 10 / 93$ & L & 0 & 7.3 & 112 & 17.0 \\
\hline 08031 & SE & $5 / 10 / 93$ & $\mathbf{L}$ & 0 & 7.2 & 103 & 16.0 \\
\hline 08033 & SE & $5 / 10 / 93$ & D & 0 & -. & -- & -- \\
\hline 08034 & SE & $5 / 10 / 93$ & D & 0 & -- & -- & -- \\
\hline 08035 & SE & $5 / 10 / 93$ & D & 0 & -. & -- & -- \\
\hline 08037 & $\mathrm{Q}$ & $5 / 10 / 93$ & $\mathrm{D}$ & 0 & -- & -. & -- \\
\hline 08039 & SE & $5 / 10 / 93$ & $\mathrm{E}$ & .01 & 6.8 & 186 & 15.0 \\
\hline 08043 & SE & $5 / 10 / 93$ & $\mathbf{L}$ & 0 & 6.8 & 128 & 18.0 \\
\hline 08045 & $\mathrm{SE}$ & $5 / 10 / 93$ & L & 0 & 6.7 & 116 & 20.5 \\
\hline 08050 & SE & $5 / 10 / 93$ & $D$ & 0 & -- & -- & - \\
\hline 08055 & $\mathrm{Q}$ & $5 / 10 / 93$ & $\mathrm{~F}$ & .04 & 7.4 & 135 & 18.0 \\
\hline 08060 & SE & $5 / 10 / 93$ & $\mathrm{D}$ & 0 & .- & -- & -- \\
\hline 08065 & $\mathrm{Q}$ & $5 / 10 / 93$ & D & 0 & -- & -- & -- \\
\hline 08070 & SE & $5 / 10 / 93$ & $D$ & 0 & -- & -- & -- \\
\hline 08075 & $\mathrm{SE} / \mathrm{A}$ & $5 / 10 / 93$ & $\mathrm{~L}$ & 0 & 6.6 & 53 & 15.5 \\
\hline 08080 & $\mathrm{SE}$ & $5 / 10 / 93$ & D & 0 & -. & -- & -- \\
\hline 08085 & SE & $5 / 10 / 93$ & $\mathrm{~L}$ & 0 & 6.6 & 56 & 18.5 \\
\hline 08090 & SE & $5 / 10 / 93$ & V & .01 & 7.2 & 159 & 14.0 \\
\hline 08095 & SE & $5 / 10 / 93$ & D & 0 & -- & -- & -- \\
\hline 08100 & $\mathrm{Q}$ & $5 / 10 / 93$ & $\mathbf{L}$ & 0 & 6.8 & 74 & 21.0 \\
\hline 08105 & SE & $5 / 10 / 93$ & $\mathrm{D}$ & 0 & -- & -- & -- \\
\hline 08107 & SE & $5 / 10 / 93$ & D & 0 & -- & -- & -- \\
\hline 08110 & SE & $5 / 10 / 93$ & $\mathrm{~L}$ & 0 & 5.8 & 167 & 19.0 \\
\hline 08115 & SE & $5 / 10 / 93$ & $\mathrm{E}$ & .02 & 6.4 & 72 & 14.5 \\
\hline 08120 & $\mathrm{Q}$ & $5 / 10 / 93$ & $\mathbf{P}$ & .03 & 7.8 & 191 & 20.5 \\
\hline 08125 & $\mathrm{Q}$ & $5 / 10 / 93$ & L & 0 & 7.6 & 183 & 20.0 \\
\hline 08130 & SE & $5 / 10 / 93$ & L & 0 & 7.5 & 182 & 20.0 \\
\hline 08135 & $\mathrm{Q}$ & $5 / 10 / 93$ & $\mathrm{~L}$ & 0 & 7.1 & 273 & 15.5 \\
\hline 08140 & SE & $5 / 10 / 93$ & D & 0 & -- & -- & -- \\
\hline 08145 & $Q$ & $5 / 10 / 93$ & $\mathrm{~L}$ & 0 & 6.3 & 76 & 14.5 \\
\hline 08150 & SE & $5 / 10 / 93$ & D & 0 & -- & -- & -. \\
\hline 08155 & $\mathrm{Q}$ & $5 / 10 / 93$ & $\mathrm{~L}$ & 0 & 7.4 & 153 & 19.5 \\
\hline 08160 & $\mathrm{Q}$ & $5 / 10 / 93$ & $\mathbf{P}$ & .03 & 7.7 & 163 & 20.0 \\
\hline 08165 & $\mathrm{Q}$ & $5 / 10 / 93$ & $\mathrm{~L}$ & 0 & 7.7 & 175 & 19.5 \\
\hline 08170 & $\mathrm{Q}$ & $5 / 10 / 93$ & L & 0 & 7.7 & 182 & 18.5 \\
\hline 08185 & $\mathrm{Q}$ & $5 / 10 / 93$ & $\mathrm{~L}$ & 0 & 7.7 & 139 & 17.5 \\
\hline 08190 & $\mathrm{Q}$ & $5 / 10 / 93$ & $\mathrm{~L}$ & 0 & 7.4 & 161 & 16.5 \\
\hline 08195 & $\mathrm{SE} / \mathrm{R}$ & $5 / 10 / 93$ & $\mathbf{L}$ & 0 & 6.7 & 180 & 14.0 \\
\hline 08200 & $\mathrm{Q}$ & $5 / 10 / 93$ & D & 0 & -. & -- & -- \\
\hline 08205 & SE & $5 / 10 / 93$ & D & 0 & -- & -- & -- \\
\hline 08210 & $\mathrm{Q}$ & $5 / 10 / 93$ & D & 0 & -- & -- & -- \\
\hline 08215 & $\mathrm{SE}$ & $5 / 10 / 93$ & D & 0 & -- & -- & -- \\
\hline 08220 & $\mathrm{Q}$ & $5 / 10 / 93$ & $\mathrm{~L}$ & 0 & 7.7 & 221 & 15.5 \\
\hline 08225 & $\mathrm{Q}$ & $5 / 10 / 93$ & $\mathbf{L}$ & 0 & 6.9 & 160 & 15.5 \\
\hline 08230 & $\mathrm{Q}$ & $5 / 10 / 93$ & $\mathrm{~L}$ & 0 & 7.3 & 154 & 15.5 \\
\hline 08235 & SE & $5 / 10 / 93$ & $\mathrm{D}$ & 0 & -- & -- & -- \\
\hline 08238 & $\mathrm{Q}$ & $5 / 10 / 93$ & $L$ & 0 & 7.6 & 133 & 22.5 \\
\hline 08240 & $\mathrm{Q}$ & $5 / 10 / 93$ & $\mathrm{~L}$ & 0 & 6.1 & 71 & 18.0 \\
\hline 08245 & SE & $5 / 10 / 93$ & $\mathrm{~L}$ & 0 & 6.6 & 56 & 14.5 \\
\hline 08250 & $\mathrm{Q}$ & $5 / 10 / 93$ & $\mathrm{~V}$ & 0.01 & 7.8 & 158 & 19.5 \\
\hline
\end{tabular}


Table 3. Discharge and water-quality data for the high base flow seepage investigation at Oak Ridge National Laboratory, April 29 through May 10, 1993--Continued

\begin{tabular}{|c|c|c|c|c|c|c|c|}
\hline $\begin{array}{c}\text { Site } \\
\text { number }\end{array}$ & $\begin{array}{l}\text { Type } \\
\text { of } \\
\text { site }\end{array}$ & $\begin{array}{c}\text { Date } \\
\text { sampled } \\
\text { (month/ } \\
\text { day/year) }\end{array}$ & Method & $\begin{array}{l}\text { Flow } \\
\left(\mathrm{ft}^{3} / 8\right)\end{array}$ & pH & $\begin{array}{c}\text { Spocific } \\
\text { conductance } \\
(\mu \mathrm{S} / \mathrm{cm})\end{array}$ & $\begin{array}{c}\text { Temperature } \\
\left({ }^{\circ} \mathrm{C}\right)\end{array}$ \\
\hline 08255 & $\mathrm{Q}$ & $5 / 10 / 93$ & V & .01 & 7.6 & 155 & 17.5 \\
\hline 08260 & $Q$ & $5 / 10 / 93$ & $\mathrm{D}$ & 0 & -- & -- & -- \\
\hline 08265 & $\mathrm{Q}$ & $5 / 10 / 93$ & $\mathrm{~L}$ & 0 & 7.1 & 82 & 16.0 \\
\hline 08270 & $\mathrm{Q}$ & $5 / 10 / 93$ & L & 0 & 6.9 & 121 & 22.5 \\
\hline 08272 & $\mathrm{SE}$ & $5 / 10 / 93$ & $\mathrm{D}$ & 0 & -. & -- & -- \\
\hline 08275 & $\mathrm{Q}$ & $5 / 10 / 93$ & $P$ & .01 & 7.7 & 168 & 18.0 \\
\hline 08280 & SE & $5 / 10 / 93$ & $\mathrm{~L}$ & 0 & 6.9 & 217 & 15.0 \\
\hline 08285 & SE & $5 / 10 / 93$ & $\mathbf{L}$ & 0 & 6.5 & 50 & 15.5 \\
\hline 08290 & $\mathrm{Q}$ & $5 / 10 / 93$ & D & 0 & -- & -- & -- \\
\hline 08295 & $\mathbf{Q}$ & $5 / 10 / 93$ & D & 0 & -- & -- & -- \\
\hline 08300 & $\mathrm{Q}$ & $5 / 10 / 93$ & D & 0 & -- & - & - \\
\hline 08305 & SE & $5 / 10 / 93$ & D & 0 & -- & -- & -- \\
\hline 08310 & $\mathbf{Q}$ & $5 / 10 / 93$ & $\mathbf{P}$ & .01 & 7.9 & 183 & 21.5 \\
\hline 08315 & $\mathbf{Q}$ & $5 / 10 / 93$ & D & 0 & -- & -- & -- \\
\hline 08320 & Q & $5 / 10 / 93$ & L & 0 & 7.4 & 131 & 18.5 \\
\hline 08330 & $\mathrm{Q}$ & $5 / 10 / 93$ & $\mathbf{L}$ & 0 & 7.4 & 137 & 17.0 \\
\hline 08335 & $\mathrm{Q}$ & $5 / 10 / 93$ & L & 0 & 7.7 & 121 & 19.0 \\
\hline 08340 & $\mathrm{Q}$ & $5 / 10 / 93$ & D & 0 & -- & -- & - \\
\hline 08350 & SE & $5 / 10 / 93$ & D & 0 & -- & -- & -- \\
\hline 08355 & $\mathrm{Q}$ & $5 / 10 / 93$ & $\mathbf{L}$ & 0 & 7.4 & 92 & 19.0 \\
\hline 08360 & SE & $5 / 10 / 93$ & D & 0 & -- & -- & -- \\
\hline 08365 & $\mathrm{SE} / \mathrm{R}$ & $5 / 10 / 93$ & $\mathrm{~L}$ & 0 & 6.6 & 48 & 14.5 \\
\hline 08375 & $\mathrm{Q}$ & $5 / 10 / 93$ & $\mathrm{P}$ & .01 & 8.0 & 229 & 21.0 \\
\hline 08380 & $\mathrm{Q}$ & $5 / 10 / 93$ & $P$ & .02 & 7.5 & 253 & 24.0 \\
\hline 08385 & $\mathrm{Q}$ & $5 / 10 / 93$ & $\mathrm{~L}$ & 0 & 7.5 & 76 & 19.0 \\
\hline 08390 & SE & $5 / 10 / 93$ & D & 0 & -- & -- & -- \\
\hline 08395 & SE & $5 / 10 / 93$ & L & 0 & 6.3 & 51 & 15.5 \\
\hline 08400 & $\mathrm{SE}$ & $5 / 10 / 93$ & L & 0 & 6.6 & 51 & 14.5 \\
\hline 09025 & $\mathrm{Q}$ & $5 / 7 / 93$ & $\mathrm{~L}$ & 0 & 6.5 & 80 & 14.5 \\
\hline 09030 & SE & $5 / 7 / 93$ & D & 0 & -- & -. & -- \\
\hline 09035 & $\mathrm{SE}$ & $5 / 7 / 93$ & D & 0 & - & -- & - \\
\hline 09040 & Q & $5 / 7 / 93$ & L & 0 & 7.8 & 162 & 18.0 \\
\hline 09045 & $\mathrm{SE}$ & $5 / 7 / 93$ & D & 0 & -- & -- & -- \\
\hline 09050 & $\mathrm{SE} / \mathrm{R}$ & $5 / 7 / 93$ & $\mathrm{~L}$ & 0 & 7.4 & 471 & 17.0 \\
\hline 09055 & SE & $5 / 7 / 93$ & D & 0 & -. & -- & -- \\
\hline 09060 & SE & $5 / 7 / 93$ & $\mathbf{L}$ & 0 & 6.8 & 245 & 13.0 \\
\hline 09065 & SE & $5 / 7 / 93$ & D & 0 & -- & -- & -- \\
\hline 09070 & SE & $5 / 7 / 93$ & $P$ & 0.04 & 8.2 & 412 & 15.5 \\
\hline 09075 & $\mathrm{Q}$ & $5 / 7 / 93$ & $P$ & .04 & 8.0 & 419 & 15.5 \\
\hline 09080 & Q & $5 / 7 / 93$ & $\mathrm{~L}$ & 0 & 7.8 & 330 & 14.5 \\
\hline 09085 & $\mathrm{SE}$ & $5 / 7 / 93$ & D & 0 & -- & -- & -- \\
\hline 09090 & $\mathrm{Q}$ & $5 / 7 / 93$ & $\mathrm{P}$ & .02 & 8.0 & 470 & 14.5 \\
\hline 09095 & $\mathrm{SE}$ & $5 / 7 / 93$ & $\mathrm{~L}$ & 0 & 7.5 & 483 & 12.5 \\
\hline 09100 & $\mathrm{Q}$ & $5 / 7 / 93$ & $\mathrm{~L}$ & 0 & 7.4 & 138 & 19.0 \\
\hline 09103 & SE & $5 / 7 / 93$ & D & 0 & -- & -- & -- \\
\hline 09105 & $\mathrm{Q}$ & $5 / 7 / 93$ & $\mathbf{L}$ & 0 & 7.3 & 140 & 17.0 \\
\hline 09110 & $\mathrm{SE}$ & $5 / 7 / 93$ & D & 0 & -- & -- & -- \\
\hline 09115 & $\mathrm{Q}$ & $5 / 7 / 93$ & L & 0 & 7.0 & 235 & 16.5 \\
\hline 09125 & $\mathbf{Q}$ & $5 / 7 / 93$ & $\mathbf{P}$ & .05 & 8.4 & 382 & 20.0 \\
\hline 09130 & $\mathrm{Q}$ & $5 / 7 / 93$ & $\mathbf{P}$ & .05 & 8.3 & 390 & 19.5 \\
\hline 09135 & $\mathrm{Q}$ & $5 / 7 / 93$ & $\mathbf{P}$ & .04 & 8.3 & 410 & 19.0 \\
\hline 09140 & SP & $5 / 7 / 93$ & $\mathbf{P}$ & .03 & 7.5 & 436 & 13.0 \\
\hline 09375 & $\mathrm{Q}$ & $5 / 7 / 93$ & $\mathbf{P}$ & .01 & 7.8 & 351 & 16.5 \\
\hline
\end{tabular}


Table 3. Discharge and water-quality data for the high base flow seepage investigation at Oak Ridge National Laboratory, April 29 through May 10, 1993--Continued

\begin{tabular}{|c|c|c|c|c|c|c|c|}
\hline $\begin{array}{c}\text { Site } \\
\text { number }\end{array}$ & $\begin{array}{l}\text { Type } \\
\text { of } \\
\text { site }\end{array}$ & $\begin{array}{c}\text { Date } \\
\text { sampled } \\
\text { (month/ } \\
\text { day/year) }\end{array}$ & Method & $\begin{array}{l}\text { Flow } \\
\left(f^{3} / s\right)\end{array}$ & pH & $\begin{array}{c}\text { Specific } \\
\text { conductance } \\
(\mu \mathrm{S} / \mathrm{cm})\end{array}$ & $\begin{array}{c}\text { Temperature } \\
\left({ }^{\circ} \mathrm{C}\right)\end{array}$ \\
\hline 09385 & $Q$ & $5 / 7 / 93$ & L & 0 & 7.5 & 399 & 16.0 \\
\hline 09390 & $\mathrm{SE} / \mathrm{A}$ & $5 / 7 / 93$ & D & 0 & -- & -- & -- \\
\hline 09395 & SE & $5 / 7 / 93$ & D & 0 & -- & -- & -- \\
\hline 09400 & SE & $5 / 7 / 93$ & D & 0 & -- & -- & -- \\
\hline 10005 & $\mathrm{Q}$ & $5 / 10 / 93$ & L & 0 & 7.4 & 156 & 15.5 \\
\hline 10010 & $\mathrm{Q}$ & $5 / 10 / 93$ & $\mathrm{~L}$ & 0 & 7.2 & 152 & 14.5 \\
\hline 10015 & $\mathrm{Q}$ & $5 / 10 / 93$ & D & 0 & -- & -- & - \\
\hline 10020 & SE & $5 / 10 / 93$ & D & 0 & -- & -- & -- \\
\hline 10170 & $\mathrm{Q}$ & $5 / 10 / 93$ & V & .04 & 8.0 & 464 & 16.5 \\
\hline 10175 & SE & $5 / 10 / 93$ & L & 0 & 7.2 & 652 & 15.0 \\
\hline 10180 & $\mathrm{Q}$ & $5 / 10 / 93$ & P & .02 & 7.9 & 476 & 16.0 \\
\hline 10185 & $\mathrm{Q}$ & $5 / 10 / 93$ & P & .02 & 8.1 & 512 & 15.5 \\
\hline 10189 & SP & $5 / 10 / 93$ & $\mathbf{P}$ & .04 & 7.2 & 545 & 13.0 \\
\hline 10190 & $\mathrm{Q}$ & $5 / 10 / 93$ & D & 0 & -- & -- & -- \\
\hline 10195 & SE & $5 / 10 / 93$ & $\mathrm{~V}$ & .01 & 7.4 & 477 & 13.0 \\
\hline 10200 & SP & $5 / 10 / 93$ & V & .01 & 7.7 & 475 & 13.5 \\
\hline 10205 & $\mathrm{Q}$ & $5 / 10 / 93$ & L & 0 & 7.5 & 288 & 17.0 \\
\hline 10220 & $\mathrm{Q}$ & $5 / 10 / 93$ & V & .01 & 8.0 & 471 & 19.5 \\
\hline 10225 & SE & $5 / 10 / 93$ & D & 0 & -- & -- & -- \\
\hline 10230 & $\mathrm{Q}$ & $5 / 10 / 93$ & L & 0 & 7.2 & 565 & 12.5 \\
\hline 10235 & SE & $5 / 10 / 93$ & D & 0 & -- & -- & -- \\
\hline 10240 & SE & $5 / 10 / 93$ & D & 0 & -- & -- & -- \\
\hline 10325 & $\mathrm{Q}$ & $5 / 10 / 93$ & V & 0.01 & 8.2 & 442 & 14.5 \\
\hline 10335 & SE & $5 / 10 / 93$ & D & 0 & -- & -- & -- \\
\hline 10340 & Q & $5 / 10 / 93$ & L & 0 & 7.3 & 226 & 15.0 \\
\hline 10350 & $\mathrm{SE}$ & $5 / 10 / 93$ & $\mathrm{D}$ & 0 & -- & -- & -- \\
\hline 10355 & $\mathrm{Q}$ & $5 / 10 / 93$ & $\mathrm{P}$ & .02 & 7.8 & 464 & 14.5 \\
\hline 10360 & $\mathrm{SE}$ & $5 / 10 / 93$ & $\mathrm{~L}$ & 0 & 8.3 & 491 & 15.0 \\
\hline 10365 & Q & $5 / 10 / 93$ & V & .01 & 7.9 & 390 & 15.5 \\
\hline 10370 & Q & $5 / 10 / 93$ & $\mathrm{~L}$ & 0 & 7.8 & 479 & 15.0 \\
\hline 10390 & $\mathrm{Q}$ & $5 / 10 / 93$ & $\mathrm{~L}$ & 0 & 7.7 & 396 & 14.0 \\
\hline 10395 & $\mathrm{SE}$ & $5 / 10 / 93$ & $\mathrm{~L}$ & 0 & 7.4 & 600 & 13.5 \\
\hline 10400 & $\mathrm{Q}$ & $5 / 10 / 93$ & L & 0 & 7.4 & 379 & 14.0 \\
\hline 10405 & Q & $5 / 10 / 93$ & $\mathbf{L}$ & 0 & 7.4 & 465 & 13.0 \\
\hline 10410 & SE & $5 / 10 / 93$ & D & 0 & -- & -- & -- \\
\hline 10430 & SE & $5 / 10 / 93$ & D & 0 & -- & - & -- \\
\hline 10450 & SE & $5 / 10 / 93$ & D & 0 & -- & -- & -- \\
\hline 10460 & SE & $5 / 10 / 93$ & D & 0 & -- & -- & -- \\
\hline 10470 & SE & $5 / 10 / 93$ & $\mathbf{L}$ & 0 & 7.3 & 587 & 13.0 \\
\hline 10475 & $\mathrm{Q}$ & $5 / 10 / 93$ & D & 0 & -- & -- & -- \\
\hline 10480 & SE & $5 / 10 / 93$ & L & 0 & 7.6 & 619 & 14.5 \\
\hline 10485 & $\mathrm{Q}$ & $5 / 10 / 93$ & L & 0 & 7.7 & 215 & 15.0 \\
\hline 10490 & $\mathrm{Q}$ & $5 / 10 / 93$ & $\mathbf{L}$ & 0 & 7.1 & 135 & 13.5 \\
\hline 10510 & SE & $5 / 10 / 93$ & L & 0 & 7.4 & 590 & 13.5 \\
\hline 10600 & Q & $5 / 10 / 93$ & $P$ & .03 & 8.1 & 429 & 15.5 \\
\hline 10605 & $\mathrm{Q}$ & $5 / 10 / 93$ & $\mathrm{P}$ & .05 & 8.0 & 460 & 15.0 \\
\hline 10607 & SE & $5 / 10 / 93$ & $\mathrm{E}$ & .02 & 8.0 & 451 & 13.5 \\
\hline 10610 & SE & $5 / 10 / 93$ & $\mathrm{E}$ & 0 & 7.4 & 439 & 13.0 \\
\hline 11005 & $\mathrm{Q}$ & $5 / 7 / 93$ & $\mathrm{P}$ & .02 & 7.5 & 484 & 16.0 \\
\hline 11010 & SP & $5 / 7 / 93$ & $\mathrm{P}$ & .09 & 7.3 & 528 & 13.5 \\
\hline 11015 & SE & $5 / 7 / 93$ & D & 0 & 7.3 & 505 & 14.5 \\
\hline 11020 & Q & $5 / 7 / 93$ & V & .01 & 8.1 & 676 & 20.5 \\
\hline
\end{tabular}


Table 3. Discharge and water-quality data for the high base flow seepage investigation at Oak Ridge National Laboratory, April 29 through May 10, 1993--Continued

\begin{tabular}{|c|c|c|c|c|c|c|c|}
\hline $\begin{array}{c}\text { Site } \\
\text { number }\end{array}$ & $\begin{array}{l}\text { Type } \\
\text { of } \\
\text { site }\end{array}$ & $\begin{array}{c}\text { Date } \\
\text { sampled } \\
\text { (month/ } \\
\text { daylyear) }\end{array}$ & Method & $\begin{array}{l}\text { Flow } \\
\left(\mathrm{tt}^{3} / \mathrm{s}\right)\end{array}$ & pH & $\begin{array}{c}\text { Specific } \\
\text { conductance } \\
(\mu \mathrm{S} / \mathrm{cm})\end{array}$ & $\begin{array}{c}\text { Temperature } \\
\left({ }^{\circ} \mathrm{C}\right)\end{array}$ \\
\hline 11025 & SP & $5 / 7 / 93$ & $\mathrm{~V}$ & .01 & 7.4 & 274 & 13.5 \\
\hline 11030 & $\mathrm{Q}$ & $5 / 7 / 93$ & $\mathrm{~L}$ & 0 & 7.8 & 1004 & 21.5 \\
\hline 11035 & $\mathrm{SE} / \mathrm{R}$ & $5 / 7 / 93$ & $\mathrm{~L}$ & 0 & 7.0 & 320 & 16.5 \\
\hline 11040 & Q & $5 / 7 / 93$ & D & 0 & -- & -- & -- \\
\hline 11045 & SE & $5 / 7 / 93$ & D & 0 & -. & -- & -- \\
\hline 11050 & $\mathrm{Q}$ & $5 / 7 / 93$ & V & .01 & 7.8 & 515 & 15.0 \\
\hline 11055 & SE & $5 / 7 / 93$ & $\mathrm{~L}$ & 0 & 7.4 & 354 & 12.5 \\
\hline 11060 & $\mathrm{Q}$ & $5 / 7 / 93$ & V & 0.01 & 7.3 & 337 & 14.5 \\
\hline 11070 & SP & $5 / 7 / 93$ & L & 0 & 8.0 & 414 & 14.5 \\
\hline 11080 & $\mathrm{Q}$ & $5 / 7 / 93$ & $\mathrm{~L}$ & 0 & 7.6 & 477 & 15.5 \\
\hline 11082 & $\mathrm{SE}$ & $5 / 7 / 93$ & L & 0 & 7.3 & 632 & 13.5 \\
\hline 11085 & $\mathrm{Q}$ & $5 / 7 / 93$ & $\mathrm{~L}$ & 0 & 7.7 & 114 & 14.5 \\
\hline 11087 & $Q$ & $5 / 7 / 93$ & $\mathrm{P}$ & .08 & 8.1 & 374 & 14.0 \\
\hline 11089 & SE & $5 / 7 / 93$ & D & 0 & -- & -- & -. \\
\hline 11093 & $\mathrm{Q}$ & $5 / 7 / 93$ & $P$ & .04 & 8.2 & 378 & 14.0 \\
\hline 11095 & $\mathrm{Q}$ & $5 / 7 / 93$ & $\mathbf{P}$ & .04 & 8.2 & 407 & 14.0 \\
\hline 11100 & SP & $5 / 7 / 93$ & V & .01 & 7.6 & 376 & 13.5 \\
\hline 11105 & $\mathrm{Q}$ & $5 / 7 / 93$ & $\mathrm{~L}$ & 0 & 7.9 & 376 & 14.5 \\
\hline 11110 & SE/R & $5 / 7 / 93$ & $\mathrm{~L}$ & 0 & 7.6 & 386 & 14.5 \\
\hline 12005 & $\mathrm{Q}$ & $5 / 2 / 93$ & $\mathbf{P}$ & .01 & 6.5 & 99 & 15.0 \\
\hline 12010 & $\mathrm{Q}$ & $5 / 2 / 93$ & $\mathbf{P}$ & .01 & 6.9 & 100 & 15.0 \\
\hline 12015 & $Q$ & $5 / 2 / 93$ & $\mathbf{P}$ & .02 & 6.8 & 73 & 15.5 \\
\hline 12020 & $\mathrm{Q}$ & $5 / 2 / 93$ & P & .01 & 6.2 & 59 & 13.5 \\
\hline 12025 & SE & $5 / 2 / 93$ & $\mathrm{~F}$ & .01 & 5.6 & 82 & 12.5 \\
\hline 12040 & $\mathrm{Q}$ & $5 / 2 / 93$ & F & .01 & 6.7 & 123 & 16.0 \\
\hline 12045 & $\mathrm{Q}$ & $5 / 2 / 93$ & $\mathrm{~L}$ & 0 & 6.4 & 161 & 14.0 \\
\hline 12090 & Q & $5 / 2 / 93$ & L & 0 & 6.8 & 191 & 14.0 \\
\hline 12095 & $\mathrm{Q}$ & $5 / 2 / 93$ & $\mathbf{P}$ & .06 & 7.1 & 219 & 16.0 \\
\hline 12100 & $\mathrm{Q}$ & $5 / 2 / 93$ & $\mathbf{P}$ & .04 & 7.3 & 230 & 15.5 \\
\hline 12105 & $\mathrm{Q}$ & $5 / 2 / 93$ & $P$ & .04 & 7.1 & 234 & 15.5 \\
\hline 12110 & $\mathrm{Q}$ & $5 / 2 / 93$ & L & 0 & 7.4 & 139 & 15.0 \\
\hline 12115 & $\mathrm{Q}$ & $5 / 2 / 93$ & $\mathbf{P}$ & .02 & 7.1 & 223 & 14.5 \\
\hline 12120 & $Q$ & $5 / 2 / 93$ & $\mathbf{P}$ & .04 & 7.4 & 191 & 14.5 \\
\hline 12125 & $\mathrm{Q}$ & $5 / 2 / 93$ & L & 0 & 7.6 & 506 & 13.0 \\
\hline 12130 & Q & $5 / 2 / 93$ & $\mathbf{P}$ & .03 & 7.1 & 197 & 14.5 \\
\hline 12132 & $Q$ & $5 / 2 / 93$ & E & .02 & 7.3 & 218 & 14.5 \\
\hline 12135 & $\mathrm{Q}$ & $5 / 2 / 93$ & E & .01 & 7.0 & 217 & 16.5 \\
\hline 12140 & $\mathrm{Q}$ & $5 / 2 / 93$ & L & 0 & 6.8 & 142 & 14.5 \\
\hline 12145 & $\mathrm{Q}$ & $5 / 2 / 93$ & $\mathrm{P}$ & .02 & 7.1 & 171 & 14.5 \\
\hline 12148 & $\mathrm{Q}$ & $5 / 2 / 93$ & L & 0 & 6.6 & 130 & 14.5 \\
\hline 12150 & SE & $5 / 2 / 93$ & $\mathrm{~L}$ & 0 & 6.9 & 119 & 13.0 \\
\hline 12155 & $\mathrm{Q}$ & $5 / 2 / 93$ & $\mathbf{P}$ & .01 & 6.7 & 163 & 15.0 \\
\hline 12160 & $\mathrm{Q}$ & $5 / 2 / 93$ & E & .01 & 6.7 & 117 & 16.0 \\
\hline 13140 & $\mathrm{Q}$ & $4 / 29 / 93$ & $\mathrm{P}$ & .11 & 7.4 & 167 & 15.5 \\
\hline 13150 & SE & $4 / 29 / 93$ & E & .01 & 5.9 & 72 & 13.0 \\
\hline 13155 & $\mathrm{Q}$ & $4 / 29 / 93$ & $\mathbf{P}$ & 0.07 & 7.2 & 202 & 15.0 \\
\hline 13160 & SP & $4 / 29 / 93$ & $\mathbf{P}$ & .06 & 7.2 & 230 & 14.0 \\
\hline 13162 & Q & $4 / 29 / 93$ & F & .04 & 6.6 & 32 & 16.0 \\
\hline 13165 & SE & $4 / 29 / 93$ & E & .01 & 5.4 & 30 & 11.5 \\
\hline 13170 & SE & $4 / 29 / 93$ & $\mathrm{E}$ & .01 & 4.9 & 25 & 12.0 \\
\hline 13175 & Q & $4 / 29 / 93$ & P & .24 & 7.5 & 142 & 16.5 \\
\hline 13180 & $\mathrm{Q}$ & $4 / 29 / 93$ & $\mathbf{P}$ & .02 & 6.6 & 80 & 16.5 \\
\hline 13185 & $Q$ & $4 / 29 / 93$ & $\mathbf{P}$ & .16 & 7.5 & 150 & 16.0 \\
\hline
\end{tabular}


Table 3. Discharge and water-quality data for the high base flow seepage investigation at Oak Ridge National Laboratory, April 29 through May 10, 1993--Continued

\begin{tabular}{|c|c|c|c|c|c|c|c|}
\hline $\begin{array}{c}\text { Site } \\
\text { number }\end{array}$ & $\begin{array}{l}\text { Type } \\
\text { of } \\
\text { site }\end{array}$ & $\begin{array}{c}\text { Date } \\
\text { sampled } \\
\text { (month/ } \\
\text { day/year) }\end{array}$ & Method & $\begin{array}{c}\text { Fiow } \\
\left(\mathrm{ft}^{3} / \mathbf{s}\right)\end{array}$ & pH & $\begin{array}{c}\text { Specific } \\
\text { conductance } \\
(\mu \mathrm{S} / \mathrm{cm})\end{array}$ & $\begin{array}{c}\text { Temperature } \\
\left({ }^{\circ} \mathrm{C}\right)\end{array}$ \\
\hline 13190 & Q & $4 / 29 / 93$ & P & .2 & 7.4 & 145 & 16.0 \\
\hline 13195 & $\mathrm{SE} / \mathbf{R}$ & $4 / 29 / 93$ & L & 0 & 6.6 & 174 & 15.0 \\
\hline 13200 & Q & $4 / 29 / 93$ & $\mathbf{P}$ & .28 & 7.4 & 149 & 15.5 \\
\hline 13205 & $\mathrm{Q}$ & $4 / 29 / 93$ & P & .05 & 7.2 & 142 & 15.0 \\
\hline 13210 & $Q$ & $4 / 29 / 93$ & $P$ & .03 & 7.3 & 116 & 13.5 \\
\hline 13215 & Q & $4 / 29 / 93$ & $P$ & .02 & 6.1 & 61 & 12.5 \\
\hline 13220 & $\mathrm{Q}$ & $4 / 29 / 93$ & $\mathrm{~L}$ & 0 & 5.4 & 65 & 12.0 \\
\hline 13225 & SE & $4 / 29 / 93$ & $\mathrm{E}$ & .01 & 5.5 & 69 & 13.0 \\
\hline 13230 & SE & $4 / 29 / 93$ & L & 0 & 5.1 & 40 & 13.0 \\
\hline 13235 & SP & $4 / 29 / 93$ & $P$ & .04 & 7.4 & 274 & 14.0 \\
\hline 13250 & $\mathrm{Q}$ & $4 / 29 / 93$ & $P$ & .20 & 7.7 & 158 & 16.5 \\
\hline 13255 & SE & $4 / 29 / 93$ & -- & -- & 5.5 & 45 & 13.5 \\
\hline 13260 & $\mathrm{Q}$ & $4 / 29 / 93$ & $\mathbf{P}$ & .10 & 7.9 & 161 & 16.0 \\
\hline 13265 & SE & $4 / 29 / 93$ & L & 0 & 5.7 & 47 & 12.0 \\
\hline 13270 & $\mathrm{Q}$ & $4 / 29 / 93$ & $\mathbf{P}$ & .06 & 7.2 & 53 & 14.0 \\
\hline 13274 & Q & $4 / 29 / 93$ & $P$ & .04 & 6.7 & 49 & 16.5 \\
\hline 13275 & $\mathrm{Q}$ & $4 / 29 / 93$ & V & .01 & 5.4 & 42 & 11.5 \\
\hline 13280 & $\mathrm{SE}$ & $4 / 29 / 93$ & D & 0 & -- & -- & -- \\
\hline 13285 & $\mathrm{Q}$ & $4 / 29 / 93$ & $\mathbf{P}$ & .01 & 6.3 & 52 & 12.5 \\
\hline 13290 & $\mathrm{Q}$ & $4 / 29 / 93$ & -- & -- & 7.2 & 72 & 14.0 \\
\hline 13295 & SE & $4 / 29 / 93$ & L & 0 & 6.1 & 76 & 14.5 \\
\hline 13300 & $\mathrm{Q}$ & $4 / 29 / 93$ & $\mathbf{P}$ & .06 & 7.6 & 196 & 17.0 \\
\hline 13305 & SE & $4 / 29 / 93$ & $\mathrm{~L}$ & 0 & 6.7 & 89 & 14.5 \\
\hline 13310 & $\mathrm{Q}$ & $4 / 29 / 93$ & $\mathbf{P}$ & .06 & 7.7 & 199 & 17.0 \\
\hline 13315 & SE & $4 / 29 / 93$ & L & 0 & 5.9 & 46 & 13.0 \\
\hline 13320 & SE & $4 / 29 / 93$ & D & 0 & -- & -- & -- \\
\hline 13326 & $\mathrm{Q}$ & $4 / 29 / 93$ & L & 0 & 7.1 & 344 & 16.0 \\
\hline 13328 & $\mathrm{SE} / \mathrm{R}$ & $4 / 29 / 93$ & L & 0 & 6.8 & 229 & 12.0 \\
\hline 13330 & Q & $4 / 29 / 93$ & $\mathbf{P}$ & .07 & 7.6 & 204 & 16.5 \\
\hline 13335 & $S E$ & $4 / 29 / 93$ & V & .01 & 6.5 & 157 & 15.0 \\
\hline 13340 & $\mathrm{Q}$ & $4 / 29 / 93$ & L & 0 & 6.0 & 193 & 12.5 \\
\hline 13345 & Q & $4 / 29 / 93$ & L & 0 & 6.5 & 31 & 14.5 \\
\hline 13350 & $\mathrm{Q}$ & $4 / 29 / 93$ & L & 0 & 5.6 & 48 & 13.5 \\
\hline 13355 & $\mathrm{Q}$ & $4 / 29 / 93$ & $\mathbf{P}$ & 0.04 & 8.0 & 242 & 15.0 \\
\hline 13358 & SE & $4 / 29 / 93$ & L & 0 & 5.9 & 93 & 14.5 \\
\hline 13360 & $\mathrm{SE} / \mathrm{A}$ & $4 / 29 / 93$ & $\mathbf{P}$ & .07 & 8.0 & 243 & 14.5 \\
\hline 13365 & $\mathrm{Q}$ & $4 / 29 / 93$ & $\mathbf{P}$ & .05 & 7.8 & 234 & 14.0 \\
\hline 13370 & SP & $4 / 29 / 93$ & $P$ & .05 & 7.5 & 273 & 14.0 \\
\hline 14005 & $\mathrm{Q}$ & $5 / 9 / 93$ & $\mathrm{~L}$ & 0 & 6.7 & 68 & 15.5 \\
\hline 14010 & SE & $5 / 9 / 93$ & D & 0 & -- & -. & -- \\
\hline 14015 & $\mathrm{Q}$ & $5 / 9 / 93$ & $\mathrm{D}$ & 0 & -- & -- & -- \\
\hline 14025 & $\mathrm{Q}$ & $5 / 9 / 93$ & D & 0 & -- & -- & -. \\
\hline 14060 & SE & $5 / 9 / 93$ & D & 0 & -- & -- & -- \\
\hline 14070 & SE & $5 / 9 / 93$ & D & 0 & -- & -- & -- \\
\hline 14075 & SE & $5 / 9 / 93$ & D & 0 & -- & -- & -- \\
\hline 14080 & SE & $5 / 9 / 93$ & D & 0 & -- & -- & -- \\
\hline 14095 & SE & $5 / 9 / 93$ & D & 0 & -- & -- & -- \\
\hline 14100 & SE & $5 / 9 / 93$ & D & 0 & -- & -- &.- \\
\hline 14110 & $\mathrm{Q}$ & $5 / 9 / 93$ & $\mathrm{~L}$ & 0 & 6.5 & 62 & 17.0 \\
\hline 14130 & $S E$ & $5 / 9 / 93$ & D & 0 & -- & -- & -- \\
\hline 14135 & $\mathrm{Q}$ & $5 / 9 / 93$ & V & .01 & 7.1 & 148 & 18.5 \\
\hline 14140 & SE & $5 / 9 / 93$ & L & 0 & 7.6 & 90 & 18.0 \\
\hline 14145 & $\mathrm{Q}$ & $5 / 9 / 93$ & V & .01 & 8 & 116 & 19.0 \\
\hline
\end{tabular}


Table 3. Discharge and water-quality data for the high base flow seepage investigation at Oak Ridge National Laboratory, April 29 through May 10, 1993--Continued

\begin{tabular}{|c|c|c|c|c|c|c|c|}
\hline $\begin{array}{c}\text { Site } \\
\text { number }\end{array}$ & $\begin{array}{l}\text { Type } \\
\text { of } \\
\text { site }\end{array}$ & $\begin{array}{l}\text { Date } \\
\text { sampled } \\
\text { (month/ } \\
\text { day/year) }\end{array}$ & Method & $\begin{array}{l}\text { Flow } \\
\left(t t^{3} / s\right)\end{array}$ & pH & $\begin{array}{c}\text { Specific } \\
\text { conductance } \\
(\mu \mathrm{S} / \mathrm{cm})\end{array}$ & $\begin{array}{c}\text { Temperature } \\
\left({ }^{\circ} \mathrm{C}\right)\end{array}$ \\
\hline
\end{tabular}

\begin{tabular}{|c|c|c|c|c|c|c|c|c|}
\hline 14160 & SE & $5 / 9 / 93$ & D & 0 & .- & -. & -- & \\
\hline 14175 & $\mathrm{Q}$ & $5 / 9 / 93$ & $P$ & .02 & 7.9 & 124 & 17.0 & \\
\hline 14180 & SE & $5 / 9 / 93$ & D & 0 & -- & -- & -- & \\
\hline 14190 & $\mathrm{Q}$ & $5 / 9 / 93$ & $\mathrm{E}$ & .01 & 7.3 & 134 & 13.0 & \\
\hline 14280 & Q & $5 / 9 / 93$ & L & 0 & 5.9 & 41 & 15.0 & \\
\hline 14285 & $\mathrm{Q}$ & $5 / 9 / 93$ & L & 0 & 6.0 & 57 & 14.0 & \\
\hline 14290 & SE & $5 / 9 / 93$ & D & 0 & - & -- & -- & \\
\hline 14295 & Q & $5 / 9 / 93$ & D & 0 & -- & -- & -- & \\
\hline 14297 & Q & $5 / 9 / 93$ & D & 0 & -- & -- & -- & \\
\hline 14305 & Q & $5 / 9 / 93$ & $P$ & .05 & 7.8 & 221 & 18.5 & \\
\hline 14310 & $\mathrm{Q}$ & $5 / 9 / 93$ & P & .03 & 8 & 223 & 18.0 & \\
\hline 14315 & SE & $5 / 9 / 93$ & D & 0 & -- & -- & -- & \\
\hline 14320 & $\mathrm{Q}$ & $5 / 9 / 93$ & P & .08 & 8.1 & 228 & 17.5 & \\
\hline 14325 & SE & $5 / 9 / 93$ & L & 0 & 6.6 & 76 & 15.5 & \\
\hline 14330 & $\mathrm{SE} / \mathrm{A}$ & $5 / 9 / 93$ & $\mathbf{P}$ & .04 & 8.1 & 237 & 16.5 & \\
\hline 14335 & Q & $5 / 9 / 93$ & $\mathbf{P}$ & 0.05 & 8.2 & 247 & 15.0 & \\
\hline 14340 & SP & $5 / 9 / 93$ & $\mathbf{P}$ & .01 & 7.3 & 219 & 13.0 & . \\
\hline 14345 & Q & $5 / 9 / 93$ & D & 0 & -- & -- & .. & 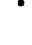 \\
\hline 14350 & $\mathrm{SE}$ & $5 / 9 / 93$ & D & 0 & -. & -- & -- & \\
\hline 14355 & Q & $5 / 9 / 93$ & L & 0 & 6.8 & 58 & 19.5 & \\
\hline 14360 & Q & $5 / 9 / 93$ & L & 0 & 7.0 & 66 & 19.0 & \\
\hline 14365 & $\mathrm{Q}$ & $5 / 9 / 93$ & L & 0 & 7.4 & 90 & 18.5 & \\
\hline 14370 & SE & $5 / 9 / 93$ & L & 0 & 6.6 & 103 & 15.5 & \\
\hline 14375 & $\mathrm{Q}$ & $5 / 9 / 93$ & L & 0 & 7.2 & 58 & 17.0 & \\
\hline 14380 & SE & $5 / 9 / 93$ & $\mathbf{L}$ & 0 & 6.3 & 105 & 15.0 & \\
\hline 14685 & $\mathrm{Q}$ & $5 / 9 / 93$ & L & 0 & 6.0 & 60 & 18.0 & \\
\hline 14690 & $\mathrm{Q}$ & $5 / 9 / 93$ & D & 0 & -- & -- & -- & \\
\hline 14695 & SE & $5 / 9 / 93$ & D & 0 & -- & -- & -- & \\
\hline 14700 & SE & $5 / 9 / 93$ & D & 0 & -- & -- & -- & \\
\hline 14705 & SE & $5 / 9 / 93$ & D & 0 & -- & -- & -- & \\
\hline 14710 & SE & $5 / 9 / 93$ & D & 0 & -- & -- & -- & \\
\hline 14715 & $\mathrm{Q}$ & $5 / 9 / 93$ & $\mathrm{~L}$ & 0 & 6.9 & 62 & 18.5 & \\
\hline 14720 & Q & $5 / 9 / 93$ & $\mathrm{~L}$ & 0 & 7.2 & 96 & 18.0 & \\
\hline 14725 & SE & $5 / 9 / 93$ & L & 0 & 6.2 & 89 & 14.0 & \\
\hline 14870 & $\mathrm{Q}$ & $5 / 9 / 93$ & $\mathrm{~L}$ & 0 & 7.5 & 94 & 19.5 & \\
\hline 14875 & SE & $5 / 9 / 93$ & $\mathrm{~L}$ & 0 & 6.9 & 95 & 15.0 & \\
\hline 15004 & $\mathrm{Q}$ & $5 / 2 / 93$ & $\mathbf{L}$ & 0 & 7.4 & 569 & 17.5 & \\
\hline 15005 & SE & $5 / 2 / 93$ & $\mathrm{~L}$ & 0 & 7.8 & 14 & 19.5 & \\
\hline 15010 & $\mathrm{Q}$ & $5 / 2 / 93$ & $\mathbf{L}$ & 0 & 7.2 & 575 & 16.5 & \\
\hline 15015 & SE & $5 / 2 / 93$ & D & 0 & -- & -- & -- & \\
\hline 15025 & Q & $5 / 2 / 93$ & $P$ & .09 & 7.7 & 526 & 14.5 & \\
\hline 15030 & SE & $5 / 2 / 93$ & $\mathrm{~L}$ & 0 & 6.9 & 655 & 14.5 & \\
\hline 15035 & $\mathrm{Q}$ & $5 / 2 / 93$ & E & .02 & 7.6 & 501 & 14.5 & \\
\hline 15038 & SE & $5 / 2 / 93$ & $\mathrm{~L}$ & 0 & 7.2 & 602 & 14.0 & \\
\hline 15045 & $\mathrm{Q}$ & $5 / 2 / 93$ & $P$ & .04 & 7.6 & 466 & 14.0 & \\
\hline 15050 & SE & $5 / 2 / 93$ & $\mathrm{~L}$ & 0 & 6.9 & 480 & 13.5 & \\
\hline 15055 & Q & $5 / 2 / 93$ & $\mathbf{L}$ & 0 & 7.2 & 488 & 14.0 & \\
\hline 15060 & $\mathrm{SE} / \mathrm{R}$ & $5 / 2 / 93$ & L & 0 & 6.7 & 498 & 13.0 & \\
\hline 15065 & Q & $5 / 2 / 93$ & E & .01 & 6.5 & 139 & 14.0 & \\
\hline 15070 & $\mathbf{Q}$ & $5 / 2 / 93$ & D & 0 & -- & -- & -- & \\
\hline 15080 & Q & $5 / 2 / 93$ & L & 0 & 7.2 & 219 & 17.0 & \\
\hline 16005 & Q & $5 / 1 / 93$ & D & 0 & -- & -- & -- & \\
\hline 16010 & Q & $5 / 1 / 93$ & D & 0 & -- & -- & -- & \\
\hline
\end{tabular}


Table 3. Discharge and water-quality data for the high base flow seepage investigation at Oak Ridge National Laboratory, April 29 through May 10, 1993--Continued

\begin{tabular}{|c|c|c|c|c|c|c|c|}
\hline $\begin{array}{c}\text { Site } \\
\text { number }\end{array}$ & $\begin{array}{l}\text { Type } \\
\text { of } \\
\text { site }\end{array}$ & $\begin{array}{c}\text { Date } \\
\text { sampied } \\
\text { (month/ } \\
\text { day/year) }\end{array}$ & Method & $\begin{array}{l}\text { Flow } \\
\left(\mathrm{ft}^{3} / \mathrm{s}\right)\end{array}$ & pH & $\begin{array}{c}\text { Speciflc } \\
\text { conductance } \\
(\mu \mathrm{S} / \mathrm{cm})\end{array}$ & $\begin{array}{c}\text { Temperature } \\
\left({ }^{\circ} \mathrm{C}\right)\end{array}$ \\
\hline 16015 & SE & $5 / 1 / 93$ & V & 0.01 & 7.5 & 70 & 13.0 \\
\hline 16020 & $\mathrm{Q}$ & $5 / 1 / 93$ & $\mathbf{P}$ & .05 & 7.9 & 211 & 15.0 \\
\hline 16023 & SE & $5 / 1 / 93$ & L & 0 & 7.8 & 444 & 21.0 \\
\hline 16025 & $\mathrm{Q}$ & $5 / 1 / 93$ & $\mathbf{P}$ & .03 & 7.5 & 182 & 15.5 \\
\hline 16030 & SP & $5 / 1 / 93$ & P & .03 & 6.4 & 147 & 12.5 \\
\hline 16035 & $\mathrm{Q}$ & $5 / 1 / 93$ & $\mathbf{P}$ & .08 & 8.0 & 287 & 15.0 \\
\hline 16040 & $\mathrm{Q}$ & $5 / 1 / 93$ & $\mathrm{P}$ & .06 & 7.6 & 272 & 15.0 \\
\hline 16045 & SE & $5 / 1 / 93$ & L & 0 & 7.1 & 354 & 15.0 \\
\hline 16050 & SP & $5 / 1 / 93$ & $\mathrm{~L}$ & 0 & 7.0 & 518 & 12.5 \\
\hline 16055 & SE & $5 / 1 / 93$ & L & 0 & 7.2 & 498 & 13.5 \\
\hline 16060 & $\mathrm{Q}$ & $5 / 1 / 93$ & $P$ & .03 & 7.7 & 234 & 14.5 \\
\hline 16065 & SE & $5 / 1 / 93$ & L & 0 & 7.3 & 338 & 13.5 \\
\hline 16075 & $\mathrm{Q}$ & $5 / 1 / 93$ & $P$ & .03 & 7.8 & 167 & 15.0 \\
\hline 16080 & $\mathrm{Q}$ & $5 / 1 / 93$ & $\mathrm{~F}$ & .04 & 7.2 & 119 & 14.0 \\
\hline 16085 & SP & $5 / 1 / 93$ & $\mathrm{~L}$ & 0 & 7.0 & 130 & 13.0 \\
\hline 16090 & $\mathrm{Q}$ & $5 / 1 / 93$ & $P$ & .01 & -- & 185 & 13.5 \\
\hline 16095 & $\mathrm{Q}$ & $5 / 1 / 93$ & $P$ & .08 & -- & 219 & 15.0 \\
\hline 16100 & SE & $5 / 1 / 93$ & L & 0 & 7.7 & 396 & 16.0 \\
\hline 16105 & $\mathrm{Q}$ & $5 / 1 / 93$ & P & .06 & 7.8 & 196 & 16.0 \\
\hline 16110 & SE & $5 / 1 / 93$ & D & 0 & -- & -- & -- \\
\hline 16115 & SE & $5 / 1 / 93$ & D & 0 & -- & -- & -- \\
\hline 16120 & SE & $5 / 1 / 93$ & L & 0 & 7.2 & 350 & 17.0 \\
\hline 16125 & SE & $5 / 1 / 93$ & D & 0 & -- & -- & -- \\
\hline 16130 & $\mathrm{Q}$ & $5 / 1 / 93$ & P & .06 & 7.7 & 144 & 15.0 \\
\hline 16135 & SP & $5 / 1 / 93$ & E & .01 & 6.8 & 107 & 12.5 \\
\hline 16140 & $\mathrm{Q}$ & $5 / 1 / 93$ & P & 2.15 & 8.1 & 246 & 17.0 \\
\hline 16145 & $Q$ & $5 / 1 / 93$ & V & .02 & 7.6 & 489 & 17.5 \\
\hline 16150 & Q & $5 / 1 / 93$ & $\mathrm{P}$ & 1.96 & 8.0 & 264 & 18.0 \\
\hline 16155 & Q & $5 / 1 / 93$ & $P$ & .61 & 7.8 & 266 & 18.5 \\
\hline 16160 & $\mathrm{Q}$ & $5 / 1 / 93$ & $\mathrm{~L}$ & 0 & 7.7 & 622 & 16.5 \\
\hline 16165 & SE & $5 / 1 / 93$ & D & 0 & -- & -- & -- \\
\hline 16170 & $\mathrm{Q}$ & $5 / 1 / 93$ & $\mathrm{~L}$ & 0 & 7.2 & 609 & 15.5 \\
\hline 16175 & Q & $5 / 1 / 93$ & $\mathrm{~L}$ & 0 & 7.0 & 466 & 16.5 \\
\hline 16180 & $\mathrm{SE}$ & $5 / 1 / 93$ & D & 0 & -- & - & -- \\
\hline 16185 & $\mathrm{SE} / \mathrm{R}$ & $5 / 1 / 93$ & $\mathrm{~L}$ & 0 & 7.9 & 687 & 17.0 \\
\hline 16300 & Q & $5 / 1 / 93$ & $P$ & 1.26 & 8.2 & 211 & 15.0 \\
\hline 16305 & Q & $5 / 1 / 93$ & $\mathrm{P}$ & .01 & 8.2 & 346 & 15.0 \\
\hline 16310 & Q & $5 / 1 / 93$ & V & .01 & 7.9 & 347 & 14.0 \\
\hline 16315 & SE & $5 / 1 / 93$ & D & 0 & -- & -- & -- \\
\hline 16320 & SE & $5 / 1 / 93$ & D & 0 & -- & -- & -- \\
\hline 16325 & $\mathrm{Q}$ & $5 / 1 / 93$ & $\mathbf{P}$ & 0.03 & 7.2 & 216 & 12.5 \\
\hline 16330 & SP & $5 / 1 / 93$ & $\mathbf{L}$ & 0 & 6.7 & 268 & 11.5 \\
\hline 16335 & SE & $5 / 1 / 93$ & D & 0 & -- & -- & -- \\
\hline 16340 & SP & $5 / 1 / 93$ & D & 0 & -- & -- & -- \\
\hline 16345 & SE & $5 / 1 / 93$ & $\mathrm{~L}$ & 0 & -- & -- & -- \\
\hline 17005 & $\mathrm{Q}$ & $5 / 3 / 93$ & $\mathbf{P}$ & 1.12 & 7.9 & 276 & 13.5 \\
\hline 17007 & SE & $5 / 3 / 93$ & L & 0 & 7.4 & 143 & 13.5 \\
\hline 17010 & SP & $5 / 3 / 93$ & $\mathbf{P}$ & .06 & 7.3 & 193 & 13.0 \\
\hline 17015 & SE & $5 / 3 / 93$ & $\mathbf{P}$ & .04 & 7.8 & 148 & 13.5 \\
\hline 17020 & Q & $5 / 3 / 93$ & $P$ & .81 & 7.9 & 285 & 13.5 \\
\hline 17025 & $\mathrm{SP}$ & $5 / 3 / 93$ & $\mathrm{~V}$ & .01 & 7.4 & 237 & 13.5 \\
\hline 17030 & SE & $5 / 3 / 93$ & $P$ & .04 & 7.8 & 171 & 14.0 \\
\hline
\end{tabular}


Table 3. Discharge and water-quality data for the high base flow seepage investigation at Oak Ridge National Laboratory, April 29 through May 10, 1993--Continued

\begin{tabular}{|c|c|c|c|c|c|c|c|}
\hline $\begin{array}{c}\text { Site } \\
\text { number }\end{array}$ & $\begin{array}{l}\text { Type } \\
\text { of } \\
\text { site }\end{array}$ & $\begin{array}{c}\text { Date } \\
\text { sampled } \\
\text { (month/ } \\
\text { day/year) }\end{array}$ & Method & $\begin{array}{l}\text { Flow } \\
\left(t^{3} / 8\right)\end{array}$ & pH & $\begin{array}{c}\text { Speclific } \\
\text { conductance } \\
(\mu \mathrm{S} / \mathrm{cm})\end{array}$ & $\begin{array}{c}\text { Temperature } \\
\left({ }^{\circ} \mathrm{C}\right)\end{array}$ \\
\hline 17035 & $Q$ & $5 / 3 / 93$ & $\mathrm{P}$ & .92 & 7.9 & 210 & 13.0 \\
\hline 17038 & $\mathrm{Q}$ & $5 / 3 / 93$ & $P$ & .04 & 7.6 & 121 & 14.0 \\
\hline 17040 & $\mathrm{Q}$ & $5 / 3 / 93$ & $P$ & .06 & 7.4 & 124 & 13.5 \\
\hline 17050 & SE & $5 / 3 / 93$ & $\mathbf{L}$ & 0 & 6.1 & 56 & 13.0 \\
\hline 17055 & SE/A & $5 / 3 / 93$ & $\mathbf{V}$ & .02 & 6.9 & 63 & 13.0 \\
\hline 17060 & $\mathrm{Q}$ & $5 / 3 / 93$ & $P$ & .64 & 7.8 & 292 & 13.5 \\
\hline 17065 & $\mathrm{Q}$ & $5 / 3 / 93$ & $\mathbf{P}$ & .05 & 7.7 & 393 & 13.5 \\
\hline 17070 & SE/R & $5 / 3 / 93$ & $P$ & .01 & 7.1 & 370 & 12.5 \\
\hline 17075 & $\mathrm{Q}$ & $5 / 3 / 93$ & $\mathbf{P}$ & .62 & 7.8 & 287 & 14.0 \\
\hline 17080 & $\mathrm{Q}$ & $5 / 3 / 93$ & $P$ & .59 & 7.8 & 290 & 13.5 \\
\hline 17085 & SE/R & $5 / 3 / 93$ & $\mathbf{E}$ & .02 & 7.8 & 407 & 13.0 \\
\hline 17090 & $\mathrm{Q}$ & $5 / 3 / 93$ & $\mathbf{P}$ & .38 & 7.2 & 281 & 13.5 \\
\hline 17095 & SE & $5 / 3 / 93$ & D & 0 & -- & -- & -- \\
\hline 17100 & $\mathrm{Q}$ & $5 / 3 / 93$ & $P$ & .43 & 7.6 & 275 & 13.5 \\
\hline 17105 & $\mathrm{Q}$ & $5 / 3 / 93$ & $P$ & .34 & 7.6 & 274 & 13.5 \\
\hline 17110 & $\mathrm{Q}$ & $5 / 3 / 93$ & $P$ & .35 & 7.4 & 272 & 13.5 \\
\hline 17115 & SP & $5 / 3 / 93$ & $\mathbf{P}$ & .04 & 5.9 & 286 & 13.0 \\
\hline 17120 & $\mathrm{Q}$ & $5 / 3 / 93$ & $\mathbf{P}$ & .15 & 7.4 & 245 & 13.5 \\
\hline 17125 & $\mathrm{Q}$ & $5 / 3 / 93$ & $\mathbf{P}$ & .11 & 7.9 & 245 & 13.5 \\
\hline 17130 & $\mathrm{Q}$ & $5 / 3 / 93$ & $\mathbf{P}$ & .19 & 8.0 & 246 & 13.5 \\
\hline 17135 & Q & $5 / 3 / 93$ & $\mathbf{P}$ & .31 & 7.8 & 243 & 13.5 \\
\hline 17140 & $\mathbf{Q}$ & $5 / 3 / 93$ & $\mathbf{P}$ & .34 & 7.2 & 242 & 13.5 \\
\hline 17145 & $\mathrm{Q}$ & $5 / 3 / 93$ & $\mathbf{P}$ & .32 & 7.1 & 234 & 13.5 \\
\hline 18012 & $\mathrm{Q}$ & $4 / 30 / 93$ & V & .01 & 7.0 & 137 & 12.5 \\
\hline 18045 & $\mathrm{Q}$ & $4 / 30 / 93$ & $\mathbf{P}$ & 0.59 & 7.9 & 272 & 12.5 \\
\hline 18050 & $\mathrm{Q}$ & $4 / 30 / 93$ & $\mathbf{P}$ & .48 & 7.9 & 310 & 12.5 \\
\hline 18055 & $\mathrm{Q}$ & $4 / 30 / 93$ & V & .01 & 7.0 & 154 & 12.5 \\
\hline 18060 & $\mathrm{Q}$ & $4 / 30 / 93$ & $\mathbf{P}$ & .64 & 8.1 & 286 & 13.0 \\
\hline 18065 & $\mathrm{Q}$ & $4 / 30 / 93$ & $\mathbf{P}$ & .1 & 7.6 & 239 & 13.0 \\
\hline 18070 & $\mathrm{Q}$ & $4 / 30 / 93$ & V & .10 & 7.3 & 238 & 12.5 \\
\hline 18075 & $\mathrm{Q}$ & $4 / 30 / 93$ & V & .01 & 7.4 & 248 & 12.5 \\
\hline 18080 & SE & $4 / 30 / 93$ & $\mathbf{L}$ & 0 & 6.7 & 180 & 13.0 \\
\hline 18085 & $Q$ & $4 / 30 / 93$ & $\mathbf{P}$ & .06 & 7.3 & 260 & 13.0 \\
\hline 18090 & $\mathrm{Q}$ & $4 / 30 / 93$ & $\mathbf{P}$ & .05 & 7.5 & 226 & 16.5 \\
\hline 18095 & $\mathrm{Q}$ & $4 / 30 / 93$ & $\mathbf{L}$ & 0 & 6.6 & 185 & 13.0 \\
\hline 18100 & SE & $4 / 30 / 93$ & $\mathbf{L}$ & 0 & 6.6 & 196 & 13.5 \\
\hline 18105 & $\mathrm{Q}$ & $4 / 30 / 93$ & $P$ & .01 & 6.8 & 190 & 13.0 \\
\hline 18110 & SE & $4 / 30 / 93$ & D & 0 & -- & -- & -- \\
\hline 18115 & $\mathrm{Q}$ & $4 / 30 / 93$ & $P$ & .01 & 6.8 & 188 & 12.5 \\
\hline 18120 & SE & $4 / 30 / 93$ & $\mathrm{~L}$ & 0 & 6.6 & 172 & 13.5 \\
\hline 18125 & $\mathrm{Q}$ & $4 / 30 / 93$ & $F$ & .04 & 7.4 & 263 & 13.0 \\
\hline 18130 & SP & $4 / 30 / 93$ & $\mathrm{E}$ & .04 & 7.5 & 273 & 13.0 \\
\hline 18135 & SE & $4 / 30 / 93$ & $\mathrm{~L}$ & 0 & 6.9 & 124 & 14.0 \\
\hline 18140 & $Q$ & $4 / 30 / 93$ & $\mathbf{R}$ & .38 & 8.0 & 289 & 14.0 \\
\hline 18145 & $\mathrm{Q}$ & $4 / 30 / 93$ & $P$ & .09 & 8.1 & 408 & 13.5 \\
\hline 18150 & $\mathrm{Q}$ & $4 / 30 / 93$ & $P$ & .33 & 8.1 & 264 & 15.5 \\
\hline 18155 & $\mathrm{Q}$ & $4 / 30 / 93$ & $P$ & .02 & 7.5 & 124 & 14.5 \\
\hline 18160 & $\mathrm{Q}$ & $4 / 30 / 93$ & $P$ & .2 & 8.1 & 350 & 16.5 \\
\hline 18165 & $\mathrm{Q}$ & $4 / 30 / 93$ & $\mathrm{E}$ & .01 & 7.1 & 346 & 12.5 \\
\hline 18170 & $\mathrm{Q}$ & $4 / 30 / 93$ & $\mathbf{P}$ & .06 & 8.0 & 289 & 12.5 \\
\hline 18175 & $\mathrm{Q}$ & $4 / 30 / 93$ & D & 0 & - & -- & -- \\
\hline 18195 & $\mathrm{Q}$ & $4 / 30 / 93$ & $\mathbf{P}$ & .04 & 7.6 & 352 & 14.0 \\
\hline 18200 & $\mathrm{Q}$ & $4 / 30 / 93$ & D & 0 & -- & -- & -- \\
\hline
\end{tabular}


Table 3. Discharge and water-quality data for the high base flow seepage investigation at Oak Ridge National Laboratory, April 29 through May 10, 1993--Continued

\begin{tabular}{|c|c|c|c|c|c|c|c|}
\hline $\begin{array}{c}\text { Site } \\
\text { number }\end{array}$ & $\begin{array}{l}\text { Type } \\
\text { of } \\
\text { site }\end{array}$ & $\begin{array}{c}\text { Date } \\
\text { sampled } \\
\text { (month/ } \\
\text { day/year) }\end{array}$ & Method & $\begin{array}{l}\text { Flow } \\
\left(\mathrm{ft}^{3} / \mathrm{s}\right)\end{array}$ & pH & $\begin{array}{c}\text { Specific } \\
\text { conductance } \\
(\mu \mathrm{S} / \mathrm{cm})\end{array}$ & $\begin{array}{c}\text { Temperature } \\
\left({ }^{\circ} \mathrm{C}\right)\end{array}$ \\
\hline 18205 & SE & $4 / 30 / 93$ & L & 0 & 5.7 & 50 & 13.0 \\
\hline 18210 & $\mathrm{Q}$ & $4 / 30 / 93$ & P & .05 & 7.6 & 321 & 13.5 \\
\hline 18215 & $Q$ & $4 / 30 / 93$ & $P$ & .17 & 8.2 & 377 & 13.0 \\
\hline 18220 & $\mathrm{Q}$ & $4 / 30 / 93$ & $P$ & .12 & 7.6 & 199 & 11.5 \\
\hline 18225 & $\mathrm{Q}$ & $4 / 30 / 93$ & $P$ & .12 & 7.6 & 200 & 11.5 \\
\hline 18230 & Q & $4 / 30 / 93$ & L & 0 & 6.5 & 87 & 11.5 \\
\hline 18235 & $\mathrm{SE}$ & $4 / 30 / 93$ & D & 0 & - & -- & -- \\
\hline 18240 & $\mathrm{Q}$ & $4 / 30 / 93$ & $P$ & .16 & 7.9 & 204 & 13.0 \\
\hline 18245 & $Q$ & $4 / 30 / 93$ & $\mathbf{P}$ & .14 & 7.7 & 201 & 16.5 \\
\hline 18250 & $\mathrm{Q}$ & $4 / 30 / 93$ & $\mathbf{P}$ & 0.02 & 7.7 & 214 & 15.5 \\
\hline 18255 & $Q$ & $4 / 30 / 93$ & E & .01 & 7.4 & 237 & 14.5 \\
\hline 18260 & $\mathrm{SE}$ & $4 / 30 / 93$ & L & 0 & 6.3 & 146 & 13.5 \\
\hline 18265 & $\mathrm{Q}$ & $4 / 30 / 93$ & L & 0 & 6.6 & 212 & 14.0 \\
\hline 18270 & Q & $4 / 30 / 93$ & V & .01 & 6.6 & 226 & 17.5 \\
\hline 18275 & $\mathrm{Q}$ & $4 / 30 / 93$ & $P$ & .11 & 7.1 & 164 & 16.5 \\
\hline 18276 & $\mathrm{Q}$ & $4 / 30 / 93$ & $\mathrm{P}$ & .11 & 7.2 & 177 & 17.0 \\
\hline 18277 & SP & $4 / 30 / 93$ & $P$ & .04 & 6.9 & 201 & 15.5 \\
\hline 18280 & $\mathrm{Q}$ & $4 / 30 / 93$ & $\mathrm{P}$ & .06 & 6.8 & 183 & 16.0 \\
\hline 18285 & Q & $4 / 30 / 93$ & $P$ & .04 & 6.7 & 175 & 17.5 \\
\hline 18290 & $\mathrm{Q}$ & $4 / 30 / 93$ & L & 0 & 7.4 & 28 & 12.5 \\
\hline 18295 & SE & $4 / 30 / 93$ & $\mathrm{~L}$ & 0 & 7.2 & 298 & 12.5 \\
\hline 18300 & $\mathrm{Q}$ & $4 / 30 / 93$ & $P$ & .06 & 7.3 & 171 & 13.0 \\
\hline 18310 & $\mathrm{SE} / \mathrm{R}$ & $4 / 30 / 93$ & L & 0 & 6.6 & 203 & 13.0 \\
\hline 18315 & $\mathrm{Q}$ & $4 / 30 / 93$ & $P$ & .03 & 6.8 & 142 & 14.0 \\
\hline 18320 & $S E$ & $4 / 30 / 93$ & L & 0 & 6.2 & 111 & 13.5 \\
\hline 18325 & $\mathrm{Q}$ & $4 / 30 / 93$ & V & .01 & 6.6 & 130 & 13.5 \\
\hline 18330 & SE & $4 / 30 / 93$ & L & 0 & 6.0 & 102 & 13.0 \\
\hline 18335 & $\mathrm{Q}$ & $4 / 30 / 93$ & $\mathbf{P}$ & .06 & 7.0 & 197 & 17.0 \\
\hline 18340 & $\mathrm{Q}$ & $4 / 30 / 93$ & $\mathbf{P}$ & .05 & 7.2 & 152 & 17.0 \\
\hline 18345 & $\mathrm{Q}$ & $4 / 30 / 93$ & $\mathbf{P}$ & .01 & 7.1 & 194 & 16.5 \\
\hline 18348 & $\mathrm{Q}$ & $4 / 30 / 93$ & V & .01 & 7.3 & 144 & 14.5 \\
\hline 18350 & $\mathrm{Q}$ & $4 / 30 / 93$ & L & 0 & 8.2 & 198 & 22.5 \\
\hline 18355 & SE & $4 / 30 / 93$ & L & 0 & 5.6 & 54 & 14.5 \\
\hline 18370 & SE & $4 / 30 / 93$ & L & 0 & 6.5 & 215 & 14.5 \\
\hline 18375 & $\mathrm{Q}$ & $4 / 30 / 93$ & $\mathbf{P}$ & .07 & 7.2 & 133 & 15.5 \\
\hline 18380 & $Q$ & $4 / 30 / 93$ & $\mathbf{P}$ & .07 & 7.0 & 115 & 13.5 \\
\hline 18385 & $\mathrm{Q}$ & $4 / 30 / 93$ & L & 0 & 6.6 & 150 & 13.0 \\
\hline 18389 & Q & $4 / 30 / 93$ & L & 0 & 6.7 & 166 & 13.0 \\
\hline 18390 & $\mathrm{Q}$ & $4 / 30 / 93$ & v & .01 & 5.6 & 100 & 13.5 \\
\hline 18405 & $\mathrm{Q}$ & $4 / 30 / 93$ & $\mathbf{P}$ & .04 & 6.6 & 83 & 13.5 \\
\hline 18410 & $\mathrm{Q}$ & $4 / 30 / 93$ & V & .02 & 6.0 & 45 & 12.5 \\
\hline 18415 & $\mathrm{Q}$ & $4 / 30 / 93$ & L & 0 & 5.1 & 36 & 13.0 \\
\hline 18420 & SE & $4 / 30 / 93$ & L & 0 & 4.9 & 29 & 12.5 \\
\hline 18435 & SE & $4 / 30 / 93$ & L & 0 & 5.0 & 40 & 13.0 \\
\hline
\end{tabular}


Table 9. Discharge and water-quality data for the low base flow seepage investigation at Oak Ridge National Laboratory, September 8-10, 1993

[a, second site identified at the same location; Type of site: Q, stream measurement sites; SE, seep; SP, spring; /R, site associated with a seepage reach; /A, site is associated with a seepage or spring area; Methods for measurement: B, backwater conditions; D, no flowing water; E, estimation; L, less than minimum reportable discharge; $P$, pygmy meter measurement; $V$, volumetric measurement; $\mathrm{ft}^{3} / \mathrm{s}$, cubic feet per second; $\mu \mathrm{S} / \mathrm{cm}$, microsiemens per centimeter; ${ }^{\circ} \mathrm{C}$, degree Celsius; --, no data]

\begin{tabular}{|c|c|c|c|c|c|c|c|}
\hline $\begin{array}{c}\text { Site } \\
\text { number }\end{array}$ & $\begin{array}{l}\text { Type } \\
\text { of } \\
\text { site }\end{array}$ & $\begin{array}{c}\text { Date } \\
\text { sampled } \\
\text { (month/ } \\
\text { day/year) }\end{array}$ & Method & $\begin{array}{l}\text { Fiow } \\
\left(\mathrm{t}^{3} / \mathrm{s}\right)\end{array}$ & pH & $\begin{array}{c}\text { Specific } \\
\text { conductance } \\
(\mu \mathrm{S} / \mathrm{cm})\end{array}$ & $\begin{array}{l}\text { Temperature } \\
\left({ }^{\circ} \mathrm{C}\right)\end{array}$ \\
\hline 01050 & SE & 9/8/93 & D & 0 & -- & -- & -- \\
\hline 01325 & SP & $9 / 8 / 93$ & $P$ & 0.01 & 6.8 & 330 & 14.0 \\
\hline 01330 & SE & 9/8/93 & E & .01 & 7.6 & 379 & 18.0 \\
\hline 01397 & SE & 9/8/93 & D & 0 & -- & -- & -- \\
\hline 02160 & SE & $9 / 8 / 93$ & D & 0 & -- & -- & - \\
\hline 02165 & SE & 9/8/93 & D & 0 & -- & -- & -- \\
\hline 02185 & SP & 9/8/93 & D & 0 & -- & - & -- \\
\hline 02194 & SE & $9 / 8 / 93$ & D & 0 & -- & -- & -- \\
\hline 02205 & SP & 9/8/93 & D & 0 & -- & -- & -- \\
\hline 02214 & SP/A & $9 / 8 / 93$ & D & 0 & -- & -- & -- \\
\hline 02230 & $\mathrm{SE} / \mathrm{R}$ & $9 / 8 / 93$ & D & 0 & -- & -- & -. \\
\hline 02240 & SE & 9/8/93 & D & 0 & -- & -- & -- \\
\hline 02245 & SE & $9 / 8 / 93$ & D & 0 & -- & -- & -- \\
\hline 02260 & SP/A & $9 / 8 / 93$ & D & 0 & - & -- & -- \\
\hline 02266 & SE & 9/8/93 & D & 0 & -- & -- & -- \\
\hline 02305 & SP & 9/8/93 & E & .01 & 7.1 & 394 & 17.0 \\
\hline 02310 & SP & $9 / 8 / 93$ & D & 0 & -- & -- & -- \\
\hline 02330 & SP & 9/8/93 & D & 0 & - & -- & -- \\
\hline 02335 & SP & $9 / 8 / 93$ & D & 0 & -- & -- & -- \\
\hline 02356 & SE & 9/8/93 & L & 0 & 7.4 & 275 & 18.0 \\
\hline 02525 & SP & $9 / 8 / 93$ & $\mathrm{~L}$ & 0 & 7.3 & 289 & 13.0 \\
\hline 02528 & SP & $9 / 8 / 93$ & D & 0 & -- & -- & - \\
\hline 02530 & $\mathrm{Q}$ & 9/8/93 & D & 0 & -- & -- & -- \\
\hline 02557 & SE & 9/8/93 & D & 0 & -- & -- & -- \\
\hline 02559 & SP & $9 / 8 / 93$ & $\mathrm{~L}$ & 0 & 7.3 & 260 & 17.5 \\
\hline 02570 & $\mathrm{SE}$ & $9 / 8 / 93$ & $\mathrm{~L}$ & 0 & 7.6 & 269 & 18.0 \\
\hline 02620 & SE & 9/8/93 & D & 0 & -- & -- & -- \\
\hline 02625 & $\mathrm{Q}$ & $9 / 8 / 93$ & $\mathrm{D}$ & 0 & -- & -. & -- \\
\hline 02630 & SE & 9/8/93 & D & 0 & -- & -- & -- \\
\hline 02640 & SE & $9 / 8 / 93$ & D & 0 & -- & -- & -. \\
\hline 02645 & SE & 9/8/93 & D & 0 & -- & -- & -- \\
\hline 02680 & SE & $9 / 8 / 93$ & D & 0 & -- & -- & -- \\
\hline 03075 & SP & $9 / 9 / 93$ & $\mathrm{~L}$ & 0 & 7.0 & 282 & 14.5 \\
\hline 03085 & SP & $9 / 9 / 93$ & L & 0 & 6.8 & 386 & 16.5 \\
\hline 03090 & SP & $9 / 9 / 93$ & $\mathbf{P}$ & 0.02 & 7.3 & 308 & 14.5 \\
\hline 03100 & SP & 9/9/93 & $\mathbf{P}$ & .11 & 7.2 & 254 & 14.0 \\
\hline 03545 & SE & $9 / 9 / 93$ & D & 0 & -- & -- & -- \\
\hline 03550 & SP & 9/9/93 & D & 0 & -- & -- & -- \\
\hline 03565 & SP & $9 / 9 / 93$ & $\mathrm{~L}$ & 0 & 7.2 & 457 & 15.5 \\
\hline 03580 & SP & 9/9/93 & D & 0 & -- & -- & -- \\
\hline 03600 & SE & 9/9/93 & D & 0 & -- & -- & -- \\
\hline 03615 & SE & $9 / 9 / 93$ & D & 0 & -- & -- & -- \\
\hline 03620 & SE & 9/9/93 & D & 0 & -- & -- & -- \\
\hline 03645 & SE & 9/9/93 & D & 0 & -- & -- & -- \\
\hline 03672 & $\mathrm{SE}$ & 9/9/93 & D & 0 & -- & -- & -- \\
\hline 03673 & SE & 9/9/93 & D & 0 & -- & -- & -- \\
\hline
\end{tabular}


Table 9. Discharge and water-quality data for the low base flow seepage investigation at Oak Ridge National Laboratory, September 8-10, 1993--Continued

\begin{tabular}{|c|c|c|c|c|c|c|c|}
\hline $\begin{array}{c}\text { Site } \\
\text { number }\end{array}$ & $\begin{array}{l}\text { Type } \\
\text { of } \\
\text { site }\end{array}$ & $\begin{array}{c}\text { Date } \\
\text { sampled } \\
\text { (month/ } \\
\text { day/year) } \\
\end{array}$ & Method & $\begin{array}{l}\text { Flow } \\
\left(\mathrm{ft}^{3} / \mathrm{s}\right)\end{array}$ & pH & $\begin{array}{c}\text { Specific } \\
\text { conductance } \\
(\mu \mathrm{S} / \mathrm{cm})\end{array}$ & $\begin{array}{c}\text { Temperature } \\
\left({ }^{\circ} \mathrm{C}\right)\end{array}$ \\
\hline 03674 & SE & $9 / 9 / 93$ & $\mathrm{E}$ & .04 & 7.1 & 305 & 15.5 \\
\hline 03697 & $\mathrm{SE} / \mathrm{R}$ & $9 / 9 / 93$ & $\mathrm{E}$ & .01 & 7.2 & 308 & 15.0 \\
\hline 03699 & $\mathrm{SE}$ & 9/9/93 & $\mathrm{D}$ & 0 & -- & -- & -- \\
\hline 03718 & $\mathrm{SE}$ & 9/9/93 & L & 0 & 7.4 & 268 & 17.0 \\
\hline 03720 & SE & 9/9/93 & E & .01 & 7.2 & 271 & 16.0 \\
\hline 04003 & SP & $9 / 8 / 93$ & $P$ & .34 & 7.5 & 286 & 17.5 \\
\hline 04009 & SE & 9/8/93 & D & 0 & -- & -. & -- \\
\hline 04012 & SE & 9/8/93 & D & 0 & -- & -- & -- \\
\hline 04015 & SE & 9/8/93 & D & 0 & -- & -- & -- \\
\hline 04021 & SE & 9/8/93 & L & 0 & 6.9 & 279 & 16.5 \\
\hline 04024 & SP/A & $9 / 8 / 93$ & $\mathrm{D}$ & 0 & -- & -- & - \\
\hline 04027 & SE & 9/8/93 & P & .07 & 7.5 & 213 & 18.0 \\
\hline 04030 & SP & 9/8/93 & E & .01 & 6.9 & 223 & 15.5 \\
\hline 04033 & SP & $9 / 8 / 93$ & $P$ & .07 & 7.6 & 257 & 14.0 \\
\hline 05070 & SP & 9/9/93 & $\mathrm{P}$ & ${ }^{\mathrm{a}} .06$ & 7.1 & 520 & 17.5 \\
\hline 05070a & SP & $9 / 9 / 93$ & & & 7.4 & 389 & 18.5 \\
\hline 05077 & SE & 9/9/93 & D & 0 & -- & -- & -- \\
\hline 05112 & $\mathrm{SE}$ & $9 / 9 / 93$ & D & 0 & -- & -- & - \\
\hline 05115 & SP & 9/9/93 & $P$ & .02 & 7.0 & 299 & 14.0 \\
\hline 05135 & SP & $9 / 9 / 93$ & L & 0 & 6.6 & 87 & 18.0 \\
\hline 05242 & SE & 9/9/93 & D & 0 & -- & -- & -- \\
\hline 05247 & $\mathrm{SE}$ & $9 / 9 / 93$ & D & 0 & -- & -. & -- \\
\hline 05290 & SP & 9/9/93 & $\mathrm{E}$ & 0.01 & 7.0 & 264 & 13.5 \\
\hline 05295 & SE/R & 9/9/93 & L & 0 & 7.3 & 323 & 19.0 \\
\hline 05310 & SE & 9/9/93 & $\mathrm{D}$ & 0 & -- & -- & - \\
\hline 05315 & SP & 9/9/93 & L & 0 & 7.6 & 246 & 13.5 \\
\hline 05320 & SE & $9 / 9 / 93$ & $\mathrm{~L}$ & 0 & 7.6 & 258 & 14.0 \\
\hline 05325 & SP & 9/9/93 & $P$ & .01 & 7.5 & 261 & 13.5 \\
\hline 05330 & SP & 9/9/93 & L & 0 & 7.3 & 258 & 15.5 \\
\hline 05335 & SP & 9/9/93 & $\mathrm{E}$ & .01 & 7.6 & 260 & 13.5 \\
\hline 05345 & SE & 9/9/93 & $\mathrm{D}$ & 0 & -- & -- & -- \\
\hline 06010 & SP & 9/9/93 & D & 0 & -- & -- & -- \\
\hline 06045 & SE & 9/9/93 & D & 0 & -- & -- & -- \\
\hline 06048 & $\mathrm{SE}$ & 9/9/93 & D & 0 & -- & -- & -- \\
\hline 06056 & SE & 9/9/93 & D & 0 & -- & -- & -- \\
\hline 06070 & SP & 9/9/93 & D & 0 & -- & - & -- \\
\hline 06075 & SP & 9/9/93 & D & 0 & -- & -- & -- \\
\hline 06100 & SP & $9 / 9 / 93$ & D & 0 & -- & - & -. \\
\hline 07021 & SE & $9 / 9 / 93$ & $\mathrm{D}$ & 0 & - & -- & -- \\
\hline 07030 & SP & 9/9/93 & D & 0 & -- & - & -- \\
\hline 07038 & SE & 9/9/93 & D & 0 & - & -- & -- \\
\hline 07120 & SE & 9/9/93 & D & 0 & -- & -- & -- \\
\hline 07228 & SE & 9/9/93 & D & 0 & -- & -- & -- \\
\hline 07410 & SP & 9/9/93 & D & 0 & -- & -- & -- \\
\hline 07430 & SP & 9/9/93 & L & 0 & 6.8 & 137 & 20.5 \\
\hline 08020 & SE & $9 / 10 / 93$ & D & 0 & -- & -- & -- \\
\hline 08025 & SE & $9 / 10 / 93$ & L & 0 & 7.4 & 271 & 19.5 \\
\hline 08031 & $\mathrm{SE}$ & $9 / 10 / 93$ & D & 0 & -- & -- & -- \\
\hline 08039 & SE & 9/10/93 & D & 0 & -- & -- & -- \\
\hline 08043 & SE & $9 / 10 / 93$ & D & 0 & -- & -- & -- \\
\hline 08045 & $\mathrm{SE}$ & 9/10/93 & D & 0 & -- & -- & -- \\
\hline
\end{tabular}


Table 9. Discharge and water-quality data for the low base flow seepage investigation at Oak Ridge National Laboratory, September 8-10, 1993--Continued

\begin{tabular}{|c|c|c|c|c|c|c|c|}
\hline $\begin{array}{c}\text { Site } \\
\text { number }\end{array}$ & $\begin{array}{l}\text { Type } \\
\text { of } \\
\text { site }\end{array}$ & $\begin{array}{c}\text { Date } \\
\text { sampled } \\
\text { (month/ } \\
\text { day/year) }\end{array}$ & Method & $\begin{array}{l}\text { Flow } \\
\left(\mathrm{ft}^{3} / \mathrm{s}\right)\end{array}$ & pH & $\begin{array}{c}\text { Specific } \\
\text { conductance } \\
(\mu \text { S } / \mathrm{cm})\end{array}$ & $\begin{array}{c}\text { Temperature } \\
\left({ }^{\circ} \mathrm{C}\right)\end{array}$ \\
\hline 08075 & $\mathrm{SE} / \mathrm{A}$ & $9 / 10 / 93$ & $\mathrm{D}$ & 0 & -- & -- & -. \\
\hline 08085 & $\mathrm{SE}$ & $9 / 10 / 93$ & $\mathrm{D}$ & 0 & -- & -- & -. \\
\hline 08090 & SE & $9 / 10 / 93$ & $\mathrm{~L}$ & 0 & 7.2 & 188 & 15.0 \\
\hline 08110 & SE & $9 / 10 / 93$ & D & 0 & -- & -- & -. \\
\hline 08115 & SE & $9 / 10 / 93$ & $\mathrm{~L}$ & 0 & 6.7 & 82 & 18.5 \\
\hline 08130 & SE & $9 / 10 / 93$ & $\mathrm{D}$ & 0 & -- & -- & -- \\
\hline 08195 & $\mathrm{SE} / \mathrm{R}$ & $9 / 10 / 93$ & $\mathrm{D}$ & 0 & -. & -. & -- \\
\hline 08245 & SE & $9 / 10 / 93$ & $\mathrm{~L}$ & 0 & 6.8 & 120 & 18.5 \\
\hline 08265 & $\mathrm{Q}$ & $9 / 10 / 93$ & $\mathrm{D}$ & 0 & - & -- & -- \\
\hline 08280 & $S E^{b}$ & $9 / 10 / 93$ & $\mathbf{L}$ & 0 & 7.4 & 259 & 19.0 \\
\hline $08280 \mathrm{a}$ & SE & $9 / 10 / 93$ & $\mathrm{~L}$ & 0 & 7.6 & 189 & 18.0 \\
\hline 08285 & SE & $9 / 10 / 93$ & D & 0 & -- & -. & -- \\
\hline 08365 & SE/R & $9 / 10 / 93$ & $\mathrm{D}$ & 0 & -- & -- & -- \\
\hline 08395 & SE & $9 / 10 / 93$ & D & 0 & -- & -- & -- \\
\hline 08400 & SE & $9 / 10 / 93$ & $\mathrm{~L}$ & 0 & 6.8 & 59 & 18.5 \\
\hline 09050 & SE/R & $9 / 10 / 93$ & $\mathrm{D}$ & 0 & -- & -- & - \\
\hline 09060 & SE & $9 / 10 / 93$ & $\mathrm{D}$ & 0 & -- & -- & -- \\
\hline 09070 & SE & $9 / 10 / 93$ & $\mathrm{D}$ & 0 & -- & -. & -- \\
\hline 09115 & $\mathrm{Q}$ & $9 / 10 / 93$ & $\mathrm{D}$ & 0 & -- & -- & -- \\
\hline 09140 & SP & $9 / 10 / 93$ & $\mathrm{~L}$ & 0 & 7.6 & 463 & 14.5 \\
\hline 10175 & SE & $9 / 10 / 93$ & $\mathrm{D}$ & 0 & -- & -- & -- \\
\hline 10189 & SP & $9 / 10 / 93$ & $\mathrm{~L}$ & 0 & 7.8 & 537 & 18.5 \\
\hline 10195 & SE & $9 / 10 / 93$ & $\mathrm{D}$ & 0 & -- & -- & -- \\
\hline 10200 & SP & $9 / 10 / 93$ & $\mathrm{D}$ & 0 & -- & -- & -- \\
\hline 10350 & SE & $9 / 10 / 93$ & $\mathrm{D}$ & 0 & -- & -- & -- \\
\hline 10395 & SE & $9 / 10 / 93$ & $\mathrm{D}$ & 0 & -- & -- & -- \\
\hline 10470 & SE & $9 / 10 / 93$ & D & 0 & -- & -- & -- \\
\hline 10480 & SE & $9 / 10 / 93$ & D & 0 & -- & -- & -- \\
\hline 10510 & $S E$ & $9 / 10 / 93$ & $\mathrm{D}$ & 0 & -- & -- & -- \\
\hline 10607 & SE & $9 / 10 / 93$ & $\mathrm{~L}$ & 0 & 8.1 & 449 & 20.5 \\
\hline 10610 & SE & $9 / 10 / 93$ & D & 0 & -- & -- & -- \\
\hline 11010 & SP & $9 / 10 / 93$ & $\mathrm{~L}$ & 0 & 7.4 & 572 & 17.5 \\
\hline 11025 & SP & $9 / 10 / 93$ & $\mathbf{L}$ & 0 & 7.3 & 514 & 17.0 \\
\hline 11035 & SE/R & $9 / 10 / 93$ & $\mathrm{D}$ & 0 & -- & -- & -- \\
\hline 11055 & SE & $9 / 10 / 93$ & D & 0 & -- & -- & - \\
\hline 11070 & SE & $9 / 10 / 93$ & $\mathrm{D}$ & 0 & -- & -- & -- \\
\hline 11082 & SE & $9 / 10 / 93$ & D & 0 & -- & -- & -- \\
\hline 11100 & SP & $9 / 10 / 93$ & $\mathrm{~L}$ & 0 & -- & 455 & 17.5 \\
\hline 11105 & $\mathrm{Q}$ & $9 / 10 / 93$ & $\mathbf{L}$ & 0 & 7.7 & 425 & 20.0 \\
\hline 11110 & SE & $9 / 10 / 93$ & D & 0 & -- & -- & -- \\
\hline 12025 & SE & $9 / 8 / 93$ & $\mathrm{D}$ & 0 & -- & -- & -- \\
\hline 12045 & $\mathrm{Q}$ & $9 / 8 / 93$ & $\mathrm{D}$ & 0 & -- & -- & -- \\
\hline 12090 & $\mathrm{Q}$ & $9 / 8 / 93$ & $\mathrm{D}$ & 0 & -- & -- & -- \\
\hline 12125 & $\mathrm{Q}$ & $9 / 8 / 93$ & $\mathrm{D}$ & 0 & -- & -- & -- \\
\hline 12135 & $\mathrm{Q}$ & $9 / 8 / 93$ & D & 0 & -- & - & -- \\
\hline 12150 & SE & $9 / 8 / 93$ & $\mathrm{D}$ & 0 & -- & -- & -- \\
\hline 13150 & SP & $9 / 8 / 93$ & $\mathrm{D}$ & 0 & -- & -- & -- \\
\hline 13155 & $\mathrm{Q}$ & $9 / 8 / 94$ & $\mathrm{E}$ & 0.02 & 7.5 & 322 & 14.5 \\
\hline 13160 & SP & 9/8/93 & $\mathrm{E}$ & .02 & 7.2 & 286 & 14.0 \\
\hline 13165 & SE & $9 / 8 / 93$ & $\mathrm{D}$ & 0 & -- & -- & -- \\
\hline 13170 & SE & $9 / 8 / 93$ & $\mathrm{D}$ & 0 & -- & -- & -- \\
\hline
\end{tabular}


Table 9. Discharge and water-quality data for the low base flow seepage investigation at Oak Ridge National Laboratory, September 8-10, 1993--Continued

\begin{tabular}{|c|c|c|c|c|c|c|c|}
\hline $\begin{array}{c}\text { Slte } \\
\text { number }\end{array}$ & $\begin{array}{l}\text { Type } \\
\text { of } \\
\text { site }\end{array}$ & $\begin{array}{c}\text { Date } \\
\text { sampled } \\
\text { (month/ } \\
\text { day/year) }\end{array}$ & Method & $\begin{array}{l}\text { Flow } \\
\left(\mathrm{ft}^{3} / 8\right)\end{array}$ & pH & $\begin{array}{c}\text { Speclfic } \\
\text { conductance } \\
(\mu \mathrm{S} / \mathrm{cm})\end{array}$ & $\begin{array}{l}\text { Temperature } \\
\left.\text { ( }{ }^{\circ} \mathrm{C}\right)\end{array}$ \\
\hline 13195 & $\mathrm{SE} / \mathrm{R}$ & 9/8/93 & D & 0 & -- & -- & -- \\
\hline 13230 & SE & $9 / 8 / 93$ & D & 0 & -- & -- & -- \\
\hline 13235 & SP & 9/8/93 & $\mathrm{E}$ & .01 & 7.6 & 343 & 15.0 \\
\hline 13255 & SE & 9/8/93 & D & 0 & -- & -- & -- \\
\hline 13265 & SE & $9 / 8 / 93$ & D & 0 & -- & -- & -- \\
\hline 13305 & SE & $9 / 8 / 93$ & D & 0 & -- & -- & -- \\
\hline 13315 & SE & 9/8/93 & D & 0 & -- & -- & -- \\
\hline 13328 & $\mathrm{SE} / \mathrm{R}$ & 9/8/93 & D & 0 & -- & -- & -- \\
\hline 13335 & SE & $9 / 8 / 93$ & D & 0 & -- & -- & -- \\
\hline 13340 & SE & $9 / 8 / 93$ & D & 0 & -- & -- & -- \\
\hline 13350 & $\mathrm{Q}$ & 9/8/93 & D & 0 & -- & -- & -- \\
\hline 13358 & SE & 9/8/93 & D & 0 & -- & -. & -- \\
\hline 13360 & $\mathrm{SE} / \mathrm{A}$ & 9/8/93 & D & 0 & -- & -- & -- \\
\hline 13370 & SP & 9/8/93 & $\mathrm{E}$ & .02 & 7.4 & 316 & 14.5 \\
\hline 14140 & SE & $9 / 9 / 93$ & D & 0 & -- & -- & -- \\
\hline 14190 & SP & 9/9/93 & $\mathrm{L}$ & 0 & 7.2 & 188 & 17.5 \\
\hline 14325 & SE & 9/9/93 & D & 0 & -. & -- & -- \\
\hline 14330 & $\mathrm{SE} / \mathrm{A}$ & 9/9/93 & $\mathrm{L}$ & 0 & 7.9 & 262 & 23.0 \\
\hline 14340 & SP & $9 / 9 / 93$ & $\mathrm{E}$ & .02 & 8.0 & 235 & 16.0 \\
\hline 14370 & $\mathrm{SE}$ & $9 / 9 / 93$ & $\mathrm{~L}$ & 0 & 7.2 & 208 & 20.0 \\
\hline 14375 & $\mathrm{SE}$ & 9/9/93 & $\mathrm{L}$ & 0 & 6.9 & 78 & 19.0 \\
\hline 14725 & SE & $9 / 9 / 93$ & $\mathrm{~L}$ & 0 & 7.2 & 161 & 21.0 \\
\hline 14875 & SE & 9/9/93 & $\mathrm{L}$ & 0 & 7.4 & 197 & 20.0 \\
\hline 15005 & SE & $9 / 8 / 93$ & D & 0 & -- & -- & -- \\
\hline 15030 & $\mathrm{SE}$ & $9 / 8 / 93$ & $\mathrm{D}$ & 0 & -- & -- & -- \\
\hline 15038 & $\mathrm{SE}$ & $9 / 8 / 93$ & D & 0 & -- & -- & -- \\
\hline 15050 & SE & 9/8/93 & D & 0 & -- & -- & -- \\
\hline 15060 & $\mathrm{SE} / \mathrm{R}$ & 9/8/93 & D & 0 & -- & -- & -- \\
\hline 16015 & SE & $9 / 10 / 93$ & D & 0 & -- & -- & -- \\
\hline 16023 & SE & 9/10/93 & D & 0 & -- & -- & -- \\
\hline 16030 & SP & $9 / 10 / 93$ & $\mathrm{~L}$ & 0 & 7.4 & 296 & 16.0 \\
\hline 16045 & SE & $9 / 10 / 93$ & D & 0 & -- & -- & -- \\
\hline 16050 & SP & $9 / 10 / 93$ & D & 0 & -- & -- & -- \\
\hline 16055 & SE & $9 / 10 / 93$ & D & 0 & -- & -- & -- \\
\hline 16065 & SE & $9 / 10 / 93$ & D & 0 & -- & -- & -- \\
\hline 16085 & SP & $9 / 10 / 93$ & $\mathbf{L}$ & 0 & 7.4 & 180 & 18.0 \\
\hline 16100 & SE & $9 / 10 / 93$ & D & 0 & -- & -- & -- \\
\hline 16120 & SE & $9 / 10 / 93$ & D & 0 & -- & -- & -- \\
\hline 16135 & SP & $9 / 10 / 93$ & E & 0.01 & 7.6 & 141 & 14.5 \\
\hline 16185 & $\mathrm{SE} / \mathrm{R}$ & $9 / 10 / 93$ & D & 0 & -- & -- & -- \\
\hline 16330 & SP & $9 / 10 / 93$ & D & 0 & -- & -- & -- \\
\hline 16345 & SE & $9 / 10 / 93$ & D & 0 & -- & -- & -- \\
\hline 17007 & $\mathrm{SE}$ & $9 / 10 / 93$ & D & 0 & -- & -- & - \\
\hline 17010 & SP & $9 / 10 / 93$ & $\mathrm{E}$ & .03 & 7.5 & 241 & 13.5 \\
\hline 17015 & SE & $9 / 10 / 93$ & E & .01 & 7.8 & 232 & 18.0 \\
\hline 17025 & SP & $9 / 10 / 93$ & $\mathrm{~L}$ & 0 & 7.5 & 272 & 20.5 \\
\hline 17030 & SE & $9 / 10 / 93$ & $\mathrm{E}$ & .02 & 7.8 & 215 & 16.5 \\
\hline 17050 & $\mathrm{SE}$ & $9 / 10 / 93$ & D & 0 & - & -- & -- \\
\hline 17055 & $\mathrm{SE} / \mathrm{A}$ & $9 / 10 / 93$ & $\mathrm{E}$ & .01 & 7.5 & 170 & 19.5 \\
\hline 17065 & $\mathrm{Q}$ & $9 / 10 / 93$ & D & 0 & -- & -- & -- \\
\hline 17070 & SE/R & $9 / 10 / 93$ & $\mathrm{~L}$ & 0 & 7.1 & 516 & 19.5 \\
\hline
\end{tabular}


Table 9. Discharge and water-quality data for the low base flow seepage investigation at Oak Ridge National Laboratory, September 8-10, 1993--Continued

\begin{tabular}{|c|c|c|c|c|c|c|c|}
\hline $\begin{array}{c}\text { Site } \\
\text { number }\end{array}$ & $\begin{array}{l}\text { Type } \\
\text { of } \\
\text { site }\end{array}$ & $\begin{array}{c}\text { Date } \\
\text { sampled } \\
\text { (month/ } \\
\text { day/year) }\end{array}$ & Method & $\begin{array}{l}\text { Flow } \\
\left(\mathrm{ft}^{3} / \mathrm{s}\right)\end{array}$ & pH & $\begin{array}{c}\text { Specific } \\
\text { conductance } \\
(\mu \mathrm{S} / \mathrm{cm})\end{array}$ & $\begin{array}{c}\text { Temperature } \\
\left({ }^{\circ} \mathrm{C}\right)\end{array}$ \\
\hline 17085 & $\mathrm{SE} / \mathrm{R}$ & $9 / 10 / 93$ & D & 0 & -- & -- & -- \\
\hline 17115 & SP & $9 / 10 / 93$ & E & .02 & 7.6 & 324 & 16.5 \\
\hline 18100 & SE & $9 / 10 / 93$ & D & 0 & -- & .. & -- \\
\hline 18120 & SE & $9 / 10 / 93$ & $\mathrm{D}$ & 0 & .- & .- & -- \\
\hline 18130 & SP & $9 / 10 / 93$ & $\mathrm{~L}$ & 0 & 7.7 & 405 & 18.5 \\
\hline 18135 & $\mathrm{Q}$ & $9 / 10 / 93$ & D & 0 & -- & -- & -- \\
\hline 18205 & SE & $9 / 10 / 93$ & D & 0 & -- & -- & -- \\
\hline 18260 & $\mathrm{SE}$ & $9 / 10 / 93$ & D & 0 & .- & -- & -- \\
\hline 18270 & $\mathrm{Q}$ & $9 / 10 / 93$ & D & 0 & .- & -. & -. \\
\hline 18277 & SP & $9 / 10 / 93$ & $\mathrm{~L}$ & 0 & 7.2 & 404 & 18.0 \\
\hline 18385 & $\mathrm{Q}$ & $9 / 10 / 93$ & D & 0 & -- & -- & -- \\
\hline 18295 & SE & $9 / 10 / 93$ & D & 0 & -- & -- & -- \\
\hline 18330 & $\mathrm{SE}$ & $9 / 10 / 93$ & D & 0 & .- & .- & -- \\
\hline 18310 & SE & $9 / 10 / 93$ & D & 0 & -- & -- & -- \\
\hline 18320 & SE & $9 / 10 / 93$ & D & 0 & -- & -- & -- \\
\hline 18355 & $\mathrm{SE}$ & $9 / 10 / 93$ & D & 0 & .- & -- & -- \\
\hline 18370 & $\mathrm{SE}$ & $9 / 10 / 93$ & D & 0 & -. & -- & -- \\
\hline 18420 & SE & $9 / 10 / 93$ & D & 0 & -- & -- & -- \\
\hline 18435 & $\mathrm{SE}$ & $9 / 10 / 93$ & D & 0 & -- & -- & -- \\
\hline
\end{tabular}

${ }^{a}$ Combined flow from two adjacent springs.

${ }^{\mathrm{b}}$ Two adjacent seeps located a few feet apart. 